\title{
Perfect equilibrium in infinite games
}

Citation for published version (APA):

Bajoori, E. (2013). Perfect equilibrium in infinite games. [Doctoral Thesis, Maastricht University]. Maastricht University. https://doi.org/10.26481/dis.20131031eb

Document status and date:

Published: 01/01/2013

DOI:

10.26481/dis.20131031eb

Document Version:

Publisher's PDF, also known as Version of record

\section{Please check the document version of this publication:}

- A submitted manuscript is the version of the article upon submission and before peer-review. There can be important differences between the submitted version and the official published version of record.

People interested in the research are advised to contact the author for the final version of the publication, or visit the DOI to the publisher's website.

- The final author version and the galley proof are versions of the publication after peer review.

- The final published version features the final layout of the paper including the volume, issue and page numbers.

Link to publication

\footnotetext{
General rights rights.

- You may freely distribute the URL identifying the publication in the public portal. please follow below link for the End User Agreement:

www.umlib.nl/taverne-license

Take down policy

If you believe that this document breaches copyright please contact us at:

repository@maastrichtuniversity.nl

providing details and we will investigate your claim.
}

Copyright and moral rights for the publications made accessible in the public portal are retained by the authors and/or other copyright owners and it is a condition of accessing publications that users recognise and abide by the legal requirements associated with these

- Users may download and print one copy of any publication from the public portal for the purpose of private study or research.

- You may not further distribute the material or use it for any profit-making activity or commercial gain

If the publication is distributed under the terms of Article $25 \mathrm{fa}$ of the Dutch Copyright Act, indicated by the "Taverne" license above, 


\section{Perfect Equilibrium in Infinite Games}


Perfect Equilibrium in Infinite Games

(c) 2013 Elnaz Bajoori

All rights reserved. No part of this publication may be reproduced, stored in a retrieval system, or transmitted, in any form, or by any means, electronic, mechanical, photocopying, recording or otherwise, without the prior permission in writing from the author.

ISBN 00-0000-0000-00 


\section{Perfect Equilibrium in Infinite Games}

\section{Proefschrift}

ter verkrijging van de graad van doctor

aan de Universiteit Maastricht,

op gezag van Rector Magnificus,

Prof. dr. L.L.G Soete,

volgens het besluit van het College van Decanen,

in het openbaar te verdedigen

op donderdag 31 oktober 2013 om 12:00 uur

door

Elnaz Bajoori

Maastricht, March 2013 


\section{Promotor}

Prof. dr. A.J. Vermeulen

\section{Copromotors}

Dr. János Flesch

\section{Beoordelingscommissie}

Prof. dr. Jean-Jacques Herings (chair)

Prof. dr. Philip J. Reny

Dr. Frank Thuijsman

Dit onderzoek werd financieel mogelijk gemaakt door Maastricht Research School of Economics of Technology and Organizations (METEOR). 


\section{Contents}

$\begin{array}{ll}\text { Contents } & \mathbf{v}\end{array}$

Preface vii

1 Introduction 1

2 Perfect equilibrium in games with compact action spaces $\quad 7$

2.1 Introduction . . . . . . . . . . . . . . . . 7

2.2 Strategic form games . . . . . . . . . . . . . . . . . . . . 9

2.3 Perfect equilibrium . . . . . . . . . . . . . . . . . . . 11

2.4 Admissibility . . . . . . . . . . . . . . . . 15

2.5 Relations between definitions of perfect equilibrium . . . . . . . . 20

2.6 Two players . . . . . . . . . . . . . . . . 25

2.7 Invariance . . . . . . . . . . . . . . . 26

2.8 Discussion . . . . . . . . . . . . . . . . . . 27

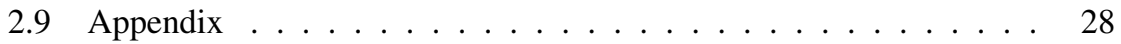

3 Behavioral perfect equilibrium in Bayesian games 35

3.1 Introduction . . . . . . . . . . . . . . 35

3.2 The model . . . . . . . . . . . . . . . . . . 37

3.3 Behavioral perfect Bayesian Nash equilibrium . . . . . . . . . . . . . 40

3.4 A second-price auction with interdependent values . . . . . . . 53

3.5 Appendix I: Proofs for the auction in Section $3.4 \ldots \ldots$. . . . . . . . 56

3.6 Appendix II: A measurability result for the weak distance . . . . . . 70

4 Distributional perfect equilibrium in Bayesian games $\mathbf{7 5}$

4.1 Introduction . . . . . . . . . . . . . . . . . 75

4.2 The model . . . . . . . . . . . . . . . . 78

4.3 Distributional perfection ....................... 82

4.4 A.e. pointwise perfection . . . . . . . . . . . . . 86

4.5 Second-price auctions with interdependent values . . . . . . . . . 88

4.6 Appendix ............................. 96

$\begin{array}{ll}\text { Mathematical tools } & 101\end{array}$

Basic concepts of measure theory _. . . . . . . . . . . 101 
Semi-product measures . . . . . . . . . . . . . . . . . . . 103

Fixed point theorems . . . . . . . . . . . . . . . . . 106

$\begin{array}{lr}\text { Bibliography } & 109\end{array}$

$\begin{array}{lr}\text { Nederlandse Samenvatting } & 113\end{array}$

$\begin{array}{lr}\text { Biography } & 117\end{array}$ 


\section{Preface}

The writing of a $\mathrm{PhD}$ thesis is a penultimate step to secure an apex academic degree. Acquiring this academic degree in the Netherlands is unique in the sense that it is a blend of studentship and employment. One gets in touch with the academic work environment while being as a student, which gives you a variety of exposures that can prove extremely helpful in ones future endeavors. During the course of my $\mathrm{PhD}$, I have learnt a lot of academic and non-academic and this would have not been possible without the help and support of many people within and outside of Maastricht University.

My sincere thanks to my supervisors Dries Vermeulen and János Flesch, whose support and patience guided me throughout my $\mathrm{PhD}$. Our regular research meetings and discussions have been very helpful to structure my thoughts and ideas into a scientifically written manuscript. Besides research, through ups and downs in the last four years, a strong friendship has been developed between us that values for me alot. Also, many thanks to Dieter Balkenborg whose special consideration towards my $\mathrm{PhD}$ application incepted my journey to the Netherlands.

I would like to thank the members of the assessment committee Jean-Jacques Herings, Philip John Reny, and Frank Thuijsman for their careful reading and for constructive comments on my manuscript. Specifically, my true appreciation to Philip Reny whose hospitality made my visit to the University of Chicago very special and productive. The research environment at the Department of Economics of the University of Chicago broadened my horizon and helped me in doing research independently.

I am very grateful to my colleagues at the Quantitative Economics Department for the friendly work environment. Special gratitude to Karin, Haydeé, and Yolanda who always patiently helped me with all the paper work I needed. A big "bedankt" to Nadine and Angie, my true friends and paranymphs on my $\mathrm{PhD}$ thesis defense. I really enjoyed the girly evenings we spent together that helped me a lot to feel at home in Maastricht. During my stay in Maastricht I have made several lovely 
friends such as Birol, Oksana, Mark, Gerg, Ruben, Anne, Artem, Martijn, Ahmad, and many others. I would like to take this opportunity to thank each and every one for all the enjoyable moments we shared.

Last but not the least, I would like to thank my family. A heartfelt appreciation to my mother whose dedication, forgiveness, and courage have always motivated me throughout my life. I have been learning a lot from her, but the most I have learnt is to believe in myself and never to give up. My father, although I have lost him in my early childhood, has been a symbol of generosity in my mind. My sisters Arezoo and Afsaneh, my brothers Omid and Amir whose kindness has brought me peace in every turbulence. Finally, special thanks to my husband for his great support and strength, and who has filled my life with love and care.

Elnaz Bajoori

August, 2013 


\section{Chapter 1}

\section{Introduction}

Formal treatment of game theory arguably starts with the work of Zermelo (1913), Borel (1921), and von Neumann (1928). But, the theory received widespread attention only after the publication of the seminal book of von Neumann and Morgenstern (1944). Game theory is the study of strategic decision making. More precisely, it is the mathematical discipline that addresses the situation where each of a group of strategic agents, the players, interact and jointly determine the outcome. Each player has certain personal preferences over the set of possible outcomes, which is measured on a given utility scale and is called the payoff of the player. A game consists of a set of players, each player's set of available choices (so-called player's action space), and each player's payoff function that is a function of all possible profiles of actions chosen by the players. In game theory we assume that players are intelligent and rational and this is common knowledge between them. A player is rational if his objective is to maximize his utility and he is intelligent if he can make any inferences about the game that we can make. A strategy for a player in a game is a decision rule that tells him which action to choose at different stages of the game.

Traditionally, games have been divided into two classes: cooperative games and noncooperative games. Noncooperative games are games in which there are no possibilities for binding agreements. A specific class of noncooperative games are normal form games. In such games, players simultaneously choose an action from their action space and subsequently each player receives a payoff depending on the resulting profile of all actions chosen by the players. Interestingly, every game can be reduced to a normal form game. In order to do this, one needs to consider the set of all possible strategies for a player as his action space. In this way, each player by choosing a strategy at the start of the game knows in advance how to act at any possible stage of the game. In this monograph, we restrict our attention to normal form games. 
A main question in game theory is how each player should act so that his partial influence on the game benefits him the most. An answer to this question is called a solution for the game. John Nash in 1950 [36] introduced the solution concept of Nash equilibrium for normal form games. A Nash equilibrium is a profile of (possibly randomized) strategies in which no player can improve his payoff by unilateral deviation. In other words, a strategy profile in equilibrium is self-enforcing. Nash proved the existence of Nash equilibrium in randomized strategies for normal form games with finite action spaces. He received the Nobel prize in 1994 for his contribution to game theory and economic science.

However, Nash equilibrium fails to select the intuitive equilibrium in some games. As an example of such cases, consider the following public good game. In this game at least two players are involved and each player has to choose an action from the set $\{y e s, n o\}$. If all of them choose yes, the public good will be provided to the players, consequently they will all enjoy a strictly positive payoff. Otherwise, they do not get anything and the payoff is zero for all players. This game contains many Nash equilibria. The most obvious Nash equilibrium is the strategy profile in which all players choose yes. However, the strategy profile where all players choose no is also a Nash equilibrium. Because, when each player believes that his opponents choose no, he is indifferent between yes and no, therefore he does not have any incentive to deviate from saying no. Nevertheless, the latter equilibrium is not intuitive. Notice that each player by choosing action yes is never worse off when compared to action no, no matter what other players do. For this reason, action no is called weakly dominated by action yes. Formally speaking, a strategy for a player is weakly dominated if it is (weakly) inferior in terms of payoffs to other choices available to him, regardless what other players choose. In the example of public good game, the strategy profile in which every player chooses no is a Nash equilibrium but actually every player chooses a strategy that is weakly dominated.

Hence, it seems necessary to refine the Nash equilibrium in order to obtain more intuitive solutions. To address this issue, in 1975 Nash's co-Nobel prize winner Selten [41] introduced trembling hand perfect equilibrium, a refinement of Nash equilibrium for games with finite action spaces that rules out equilibria where such weakly dominated strategies are played. Perfect equilibrium is a Nash equilibrium in which players choose strategies that are robust against small mistakes in the choice of strategies by opponents. In the above public good game, the only perfect equilibrium is the strategy profile in which every player says yes. The reason is that if each player takes making mistakes by his opponents serious, then he believes that all his opponents say yes at least with a small positive probability. This is enough for 
him to make yes the unique best response, as by doing so he gets a strictly positive payoff, even though with a small positive probability. But, by choosing no, he gets zero surely. The strategy profile (yes,yes,...,yes), that is robust with respect to small perturbations, is the only intuitive equilibrium in this game.

In modern economic theory, there are many game theoretic models that feature an infinity of possible choices. Some of the important examples of such games are auctions, Cournot competition models, cake division games, and location games. Since also in such models Nash equilibria may use weakly dominated strategies, in 1995 Simon and Stinchcombe [42] defined perfect equilibrium for games with infinite compact action spaces. They distinguished two different approaches. The first approach is a direct generalization of the original definition of Selten, whereas the second approach is based on finite approximations of the infinite game in question and is called the finitistic approach.

Note that in the some of the applications mentioned above, information is not always fully disclosed. Those are situations where players may have private information about the game, consequently players may be uncertain about the payoffs. Such games are called games with incomplete information. One of the interesting examples of games with incomplete information is auctions. We discuss auctions more in detail here. Consider an auction where bidders submit written bids without knowing the bid of other bidders in the auction, and in which the highest bidder wins, but the price paid is the second-highest bid. This auction is a sealed bid second-price auction and it is known as the Vickrey auction, after Vickrey who won the Nobel prize in 1996 for his research in the economic theory of incentives under asymmetric information. In such auctions each bidder knows the valuation of the object for himself, but he does not know the other bidders' valuation. There maybe many equilibria in this auction, however Vickrey showed that bidding his own valuation of the object is a weakly dominant strategy for each bidder. Now, consider a sealed bid second-price auction in which each bidder has only partial information on the valuation of the object even for himself. In this auction, bidders might not have any dominant strategy while there are multiple equilibria. Therefore, a selection tool is needed that can rule out the less intuitive equilibria. This motivates us to study perfect equilibrium as a selection criterion in games with infinite action spaces as well as in games with incomplete information.

In chapter 2, we investigate the relations between different types of perfect equilibrium, introduced by Simon and Stinchcombe. We propose an improved refinement along finitistic approach and prove its existence. Despite the fact that the finitistic approach appeals to basic intuition, our results seem to imply a severe critique 
of this approach.

In chapters 3 and 4, we extend Selten's trembling hand approach to equilibrium refinement to games with incomplete information with infinite action spaces. We develop the concept of perfect equilibrium for such games, by taking two different approaches. The difference between these two approaches is at using two distinct strategies, so-called behavioral strategies and distributional strategies. In chapter 3 , we formulate and examine several definitions of perfection based on behavioral strategies. We distinguish three definitions, using various notions of convergence of randomized strategies such as uniform, pointwise and almost everywhere pointwise convergence. In chapter 4 , we define perfection by making use of distributional strategies and prove its existence under some continuity assumptions. It should be emphasized here that the approach taken in chapter 3 is a more straightforward way of defining perfection in games with incomplete information than the one in chapter 4. However, in developing the theory, particularly in proving existence, it is difficult to work with behavioral strategies while the second approach does not have this problem. Additionally, in chapters 3 and 4 by using both approaches, we apply perfection to a class of symmetric second-price auctions with incomplete information and observe that perfect equilibrium selects a specific type of equilibrium and rules out less intuitive equilibria.

Related literature Equilibrium selection and refinement theory already have a long and steady-going tradition, arguably starting with essential equilibrium (Wu Wen-Tsün, Jiang Jia-He [47]). A Nash equilibrium in a normal form game is essential if neighboring games with slightly perturbed payoffs have equilibria close to the Nash equilibrium. Thereafter, perfect equilibrium (Selten [41]) was defined which is the main focus in this monograph. Perfect equilibrium in games with infinite action spaces is a useful tool to select Nash equilibria, for example in auction models with incomplete information, such as the ones studied in Compte and Jehiel [9] and Crémer et al. [10], where dominant strategies are not available as a selection criterion. Concrete applications of perfect equilibrium in infinite strategic form games can among others be found in Jackson et al. [16], Simon and Stinchcombe [42], and Andersson et al. [2]. A further refinement of perfect equilibrium is proper equilibrium (Myerson [35]). The idea behind proper equilibrium is that more costly mistakes occur with less probability than less costly ones. Kalai and Samet [19] by defining persistent equilibrium develop the notion of stability to neighborhood stability. All of the mentioned refinements are for finite normal form games.

Progress on refinements in the context of extensive form games are for example 
sequential equilibrium (Kreps and Wilson [22]). This concept focuses attention on the beliefs of the players at information sets not reached in the equilibrium under consideration.

Kohlberg and Mertens [21], introduce the concept of strategically stable equilibrium by motivating a number of requirements a solution concept should satisfy. Generally speaking, a stable set of equilibria is a minimal closed set of equilibria such that small perturbations of the game have equilibria close to the stable set. They fail to define a solution concept satisfying all their requirements. Mertens [28] [29] reformulates the concept of stable equilibrium. Hillas [15], shows that by changing the definition of perturbation it is possible to define a solution concept that does satisfy all of the requirements introduced by Kohlberg and Mertens. More recently Govindan and Wilson [14] work on metastable equilibria.

Theory on refinements for games with compact action spaces has been developed in, among others, Méndez et al. [27], Simon and Stinchcombe [42], and Yu [48], and Carbonell-Nicolau [7].

The above line of literature mainly concerns what Andersson et al. [2] refer to as "strategic uncertainty". In this study, they formulate a notion of strategic uncertainty for games with infinite action spaces and propose a criterion for robustness to such uncertainty.

An alternative line of research on equilibrium refinement is on "structural uncertainty" where, due to informational uncertainty, iterated deletion of weakly dominated strategies is used as a refinement technique. This approach, also known as the global games approach, was developed by Carlsson and van Damme [8], with applications in for example banking and finance (Morris and Shin [34]). 



\section{Chapter 2}

\section{Perfect equilibrium in games with compact action spaces}

\subsection{Introduction}

In 1995 Simon and Stinchcombe [42], for brevity referred to as S\&S, defined perfect equilibrium for games with an infinite compact set of actions. They distinguished two main lines of definitions of perfection. The first line is based on the notion of a completely mixed strategy, giving rise to the notions of strongly perfect and weakly perfect equilibrium. This approach can be viewed as a direct generalization of the original trembling hand definition of perfect equilibrium introduced by Selten [41]. The second line of definitions, in $S \& S$ referred to as the finitistic approach, uses the notion of an $\varepsilon$-perfect equilibrium in finite approximations of the original game. The resulting notion of perfect equilibrium is called limit-of-finite perfect equilibrium. ${ }^{1}$ They showed that the first type of perfect equilibrium only admits limit admissible strategies. Furthermore, they conjectured that the two approaches are incomparable.

In this chapter we investigate the existing relations between the various types of perfect equilibrium within the framework of strategic form games with compact action spaces and continuous payoff functions. The diagram below gives an overview of those relations between different types of perfect equilibrium that are currently known. Strongly perfect, weakly perfect, and lof (limit-of-finite) perfect equilibrium are introduced by S\&S. Strongly glof (global limit-of-finite) and weakly glof perfect equilibrium are introduced in this chapter. Limit undominated Nash equilibrium, a stronger version of limit admissibility from $\mathrm{S} \& S$, is also introduced in this chapter.

\footnotetext{
${ }^{1}$ Simon and Stinchcombe also introduce another variation on this type of perfection, called anchored perfection. We hardly ever consider this variation though.
} 


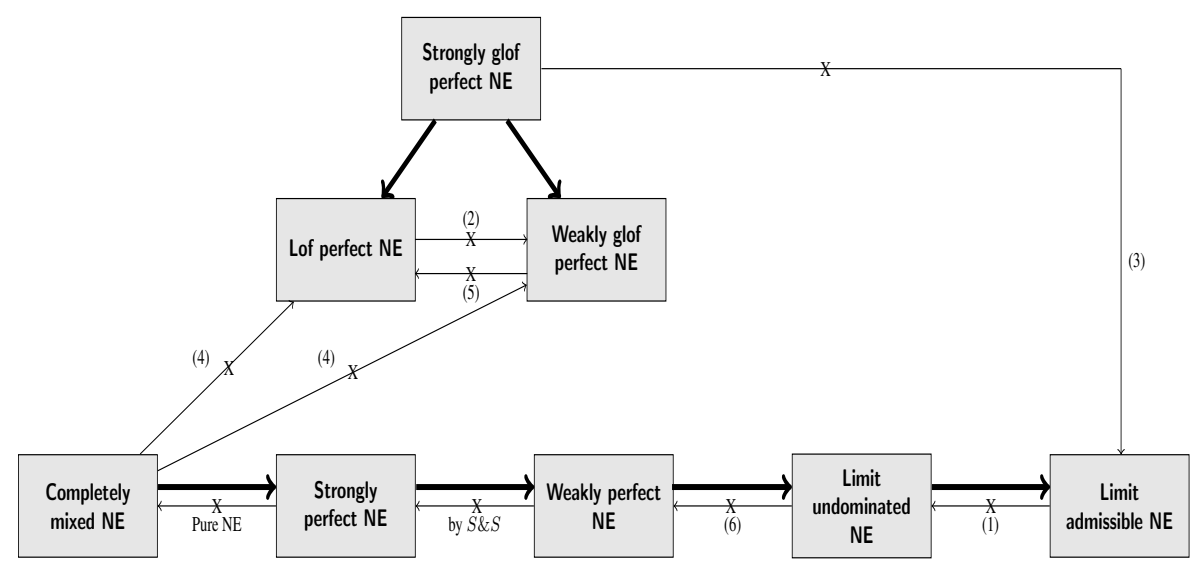

Boldface arrows indicate valid implications. For example, every strongly glof perfect equilibrium is a lof perfect equilibrium. Roman face arrows with a " $X$ " through it indicate implications that do not hold in general. Counterexamples are provided by previous studies, and examples (1) to (6) in this chapter.

Results This chapter makes two main contributions. The first contribution is the introduction of global limit-of-finite perfection, from now on referred to as glof perfection. S\&S already introduced lof perfect equilibrium as the limit of $\varepsilon$-perfect equilibria of large but finite approximations of the original game. Despite its relative straightforward intuition, lof perfect equilibrium does not always select available dominant strategies. Strongly glof perfect equilibrium is defined in the same way as lof perfect equilibrium, but with the added requirement that best responses in the approximating games are in fact best responses in the original game. Strongly glof perfect equilibrium is a stronger version of finitistic perfection than lof perfect equilibrium. Weakly glof perfect equilibrium adds to the definition of lof perfection the weak requirement that best responses in the approximating games are only close to best responses in the original game. We show existence of weakly glof perfect equilibrium. Moreover, we show that weakly (and therefore also strongly) glof perfect equilibrium does uniquely select dominant strategy Nash equilibria as soon as dominant strategies are available to a player.

Second, we provide five illustrative counterexamples. Example 2 features a lof perfect equilibrium that is not limit admissible. In fact the game in the Example encompasses Examples 2.1 and 2.4 of S\&S with the added feature that the action spaces are countable instead of the union of an interval and an isolated point. Example 3 presents a strongly glof perfect equilibrium that is not limit admissible. 
This result is in line with Example 2.4 of S\&S and our Example 2 of a lof perfect equilibrium that is not limit admissible. Example 4 verifies the conjecture of Simon and Stinchcombe that strong perfection does not imply finitistic perfection. More precisely, it is an example of a completely mixed, and therefore strongly perfect, equilibrium that is neither lof pefect (and therefore also not anchored perfect) nor weakly (and therefore also not strongly) glof perfect. Example 5 presents a weakly glof perfect equilibrium that is not lof perfect, and therefore also not strongly glof perfect.

Example 6 is a comment on the theorem of van Damme that for bimatrix games a Nash equilibrium is perfect precisely when the equilibrium strategies are undominated. This equivalence no longer holds in the more general context of two-player games with compact action spaces and continuous payoff functions. Example 6 presents a two-person game, where player 1 has two pure strategies and player 2 has a countably infinite number of pure strategies, with a Nash equilibrium in undominated strategies that is not weakly perfect. An added feature of the example is that there is another Nash equilibrium in this game that is payoff equivalent to the first Nash equilibrium, while the second Nash equilibrium is weakly perfect. Thus, weakly perfect equilibrium is not invariant, in contrast to perfect equilibrium for finite strategic form games. The example highlights the fact that for infinite compact action spaces, the topology on the action spaces starts to matter for perfection.

All our examples use countably infinite pure action spaces. We specifically chose not to use compact intervals as action spaces, in order to highlight the underlying logic of the constructions. It is nevertheless evident from the constructions that, with a bit (well, for some examples perhaps a bit more than a bit) of extra work the examples can also be transformed to counterexamples on the unit interval.

\subsection{Strategic form games}

A strategic form game (with compact action spaces) is a triplet $\Gamma=(N, A, u)$ where $N=\{1, \ldots, n\}$ is the set of the players, $A=\prod_{i \in N} A_{i}$ is the set of profiles of actions, and $u=\left(u_{1}, \ldots, u_{n}\right)$ is the vector of payoff functions. We assume for every player $i \in N$ that the sets $A_{i}$ of actions are non-empty and compact metric spaces with the metric $d_{A_{i}}$ and that the payoff functions $u_{i}: A \rightarrow \mathbf{R}$ are continuous. Note that a compact metric space is separable.

The set $\Delta\left(A_{i}\right)$ is the set of (mixed) strategies of player $i$. We identify every action $a_{i}$ with the Dirac measure $\delta\left(a_{i}\right)$, the strategy that selects $a_{i}$ with probability 1. A vector $\sigma=\left(\sigma_{i}\right)_{i \in N}$ with $\sigma_{i} \in \Delta\left(A_{i}\right)$ for all $i$ is called a strategy profile. The 
set of strategy profiles is denoted ${ }^{2}$ by $\Delta(A)$. For every strategy profile $\sigma \in \Delta(A)$ we define $u_{i}(\sigma)=\int u_{i} d \sigma$ which is continuous in $\sigma$ with respect to the weak metric $\rho^{w}$ on $\Delta(A) .^{3}$

We write $A_{-i}=\prod_{j \in N \backslash\{i\}} A_{j}$. A generic element of $\Delta\left(A_{-i}\right)$ is denoted by $\sigma_{-i}=$ $\left(\sigma_{j}\right)_{j \neq i}$. By $\left(\sigma_{-i}, \tau_{i}\right)$ or $\left(\sigma \mid \tau_{i}\right)$ we denote the strategy profile where every player $j \neq i$ adheres to strategy $\sigma_{j}$, while player $i$ uses $\tau_{i}$. A strategy $\tau_{i} \in \Delta\left(A_{i}\right)$ is called a best response of player $i$ to the strategy profile $\sigma \in \Delta(A)$ if $u_{i}\left(\sigma \mid \tau_{i}\right) \geq u_{i}\left(\sigma \mid \rho_{i}\right)$ for all $\rho_{i} \in \Delta\left(A_{i}\right)$. A strategy profile $\sigma=\left(\sigma_{i}\right)_{i \in N}$ is a Nash equilibrium if

$$
u_{i}(\sigma) \geq u_{i}\left(\sigma \mid \tau_{i}\right)
$$

for all $i$ and all $\tau_{i} \in \Delta\left(A_{i}\right)$. So, $\sigma$ is a Nash equilibrium if each $\sigma_{i}$ is a best response to $\sigma$.

For a non-empty compact subset $X_{i}$ of $A_{i}$ and strategy profile $\sigma$

$$
B R_{i}\left(\sigma \mid X_{i}\right)=\left\{\rho_{i} \in \Delta\left(X_{i}\right) \mid u_{i}\left(\sigma \mid \rho_{i}\right) \geq u_{i}\left(\sigma \mid \tau_{i}\right) \text { for all } \tau_{i} \in \Delta\left(X_{i}\right)\right\}
$$

is the set of player $i$ 's best strategies against $\sigma$ given that the pure choices of player $i$ are restricted to actions in $X_{i}$. Moreover, let $B R_{i}(\sigma)=B R_{i}\left(\sigma \mid A_{i}\right)$. An action $a_{i} \in A_{i}$ is a pure best response of player $i$ to $\sigma$ if $\delta\left(a_{i}\right) \in B R_{i}(\sigma)$. By $P B R_{i}(\sigma \mid$ $\left.X_{i}\right)$ we denote the set of those actions of player $i$ that belong to $B R_{i}\left(\sigma \mid X_{i}\right)$ and $\operatorname{PBR}_{i}(\sigma)=P B R_{i}\left(\sigma \mid A_{i}\right)$. The set $\operatorname{PBR}_{i}(\sigma)$ is non-empty by compactness of $A_{i}$ and continuity of $u_{i}$. Note that $\sigma_{i}$ is a best response to $\sigma$ if and only if it puts weight 1 on pure best responses, i.e., $\sigma_{i}\left(P B R_{i}(\sigma)\right)=1$.

We say that $\tau_{i} \in \Delta\left(A_{i}\right)$ dominates $\sigma_{i} \in \Delta\left(A_{i}\right)$ if

$$
u_{i}\left(\sigma \mid \tau_{i}\right) \geq u_{i}\left(\sigma \mid \sigma_{i}\right)
$$

for all strategy profiles $\sigma \in \Delta(A)$, and the inequality is strict for at least one strategy profile $\sigma \in \Delta(A)$. Note that we use "dominates" rather than "weakly dominates", due to the already heavy use of the adjective "weak" in this . The reader should be aware though that our use of dominance is slightly different from standard game theoretic use of this notion.

A strategy $\tau_{i}$ is dominant if $u_{i}\left(\sigma \mid \tau_{i}\right) \geq u_{i}\left(\sigma \mid \sigma_{i}\right)$ for all $\sigma \in \Delta(A)$ and $\sigma_{i} \in$ $\Delta\left(A_{i}\right)$. The set of all dominant strategies for player $i$ is denoted by $D_{i}$. Equivalently,

\footnotetext{
${ }^{2}$ With a slight abuse of notation we implicitly identify each strategy profile $\sigma=\left(\sigma_{i}\right)_{i \in N}$ with its induced probability distribution in $\Delta(A)$. Obviously not every element of $\Delta(A)$ can be obtained this way. We restrict attention to the independent elements of $\Delta(A)$ that are induced by strategy profiles.

${ }^{3}$ Billingsley [6].
} 
$D_{i}$ is the set of those strategies of player $i$ that are a best response against any strategy profile. Obviously $D_{i}$ may be empty.

If there is no $\tau_{i}$ that dominates $\sigma_{i}$, we say that $\sigma_{i}$ is undominated. We denote the set of undominated strategies of player $i$ by $U_{i}$. We write $\sigma_{i} \in L U_{i}$ if there exists a sequence $\left(\sigma_{i}^{k}\right)_{k \in \mathbf{N}}$ such that $\rho^{w}\left(\sigma_{i}^{k}, \sigma_{i}\right) \rightarrow 0$ as $k \rightarrow \infty$, and $\sigma_{i}^{k}$ is undominated for all $k{ }^{4}$ For $a_{i} \in A_{i}$ we write $a_{i} \in P U_{i}$, if $a_{i}$ is not dominated by any $\sigma_{i} \in \Delta\left(A_{i}\right)$. Following S\&S (1995), a strategy $\sigma_{i}$ is limit admissible if $\sigma_{i}\left(\overline{P U_{i}}\right)=1$, where $\overline{P U_{i}}$ denotes the closure of the set $P U_{i}$.

Every limit undominated strategy is also limit admissible. The converse does not hold though, not even for finite strategic form games.

EXAMPLE 1. In the table below the payoff matrix of player 2 is given:

$$
u_{2}=\left[\begin{array}{lll}
4 & 0 & 3 \\
0 & 4 & 3
\end{array}\right] .
$$

The strategy $\sigma_{2}=\left(\frac{1}{2}, \frac{1}{2}, 0\right)$ is limit admissible for player 2. Nevertheless, $\sigma_{2}$ is dominated by $(0,0,1) .^{5}$

\subsection{Perfect equilibrium}

Selten [41] introduced the notion of perfect equilibrium for non-cooperative extensive form games. The definition of perfect equilibrium is based on the idea that a satisfactory interpretation of equilibrium points should accommodate possible slight mistakes. Selten showed that perfect equilibrium exists for finite extensive form games with perfect recall.

Twenty years later Simon and Stinchcombe [42] provided a general treatment of perfect equilibria for infinite strategic form games with compact action spaces and continuous payoffs. S\&S discuss two distinct approaches to perfect equilibrium for infinite normal form games. In the first approach, as in Selten's original definition, players may "tremble", modeled by having players choose completely mixed strategies. Players are then required to play approximate best responses to opponents' trembling strategies. In the strong version of the first approach, a tremble assigns high probability to the set of pure best responses itself, while in the weak version a tremble is only required to assign high probability to a neighborhood of this set. The second, limit-of-finite, approach applies traditional refinements to sequences of

\footnotetext{
${ }^{4}$ In other words, the set $L U_{i}$ is the closure of the set $U_{i}$ under the weak metric. The letters $L U$ stand for limit undominated.

${ }^{5}$ See example 3 for an example of $L U_{i} \supseteqq U_{i}$. The strategy $-\infty$ is in $L U_{i}$, but not in $U_{i}$.
} 
successively larger finite approximations of the original infinite game. S\&S proved the existence of these three generalized notions of perfect equilibrium. Moreover, they showed that the set of strongly perfect equilibria is a closed, non-empty subset of the set of weakly perfect equilibria, which is a closed subset of the collection of limit admissible Nash equilibria.

Both the strong and weak versions of the first approach of S\&S use a direct generalization of the notion of completely mixed strategies in the definition of perfect equilibrium. Let $\varepsilon>0$. A completely mixed strategy profile $\sigma=\left(\sigma_{i}\right)_{i \in N}$ in $\Delta(A)$ is a strongly $\varepsilon$-perfect equilibrium if for every player $i \in N$ it holds that $\rho^{s}\left(\sigma_{i}, B R_{i}(\sigma)\right)<\varepsilon$. It is a weakly $\varepsilon$-perfect equilibrium if $\rho^{w}\left(\sigma_{i}, B R_{i}(\sigma)\right)<\varepsilon$.

Definition 2.3.1 (Simon and Stinchcombe) A strategy profile $\sigma$ is a strongly (respectively weakly) perfect equilibrium if there is a sequence $\left(\sigma^{k}\right)_{k=1}^{\infty}$ of strongly (weakly) $\frac{1}{k}$-perfect equilibria with $\rho^{w}\left(\sigma^{k}, \sigma\right) \rightarrow 0$ as $k \rightarrow \infty$.

In order to guarantee existence of limit points, the above definition only considers weak limits of $\varepsilon$-perfect equilibria. Finitistic perfection is based on a different approach where games with infinite action spaces are approximated by games with large, but still finite, action spaces. Recall that compact metric action spaces are separable.

Definition 2.3.2 (Simon and Stinchcombe) A strategy profile $\sigma=\left(\sigma_{i}\right)_{i \in N}$ is a limit-of-finite (lof) perfect equilibrium if for every player $i \in N$ there is a sequence $\left(B_{i}^{k}\right)_{k=1}^{\infty}$ of finite subsets of $A_{i}$ and a sequence $\left(\sigma_{i}^{k}\right)_{k=1}^{\infty}$ of completely mixed strategies in $\Delta\left(B_{i}^{k}\right)$ such that for every $i \in N$

[1] for every $\varepsilon>0$ there is a $K \in \mathbf{N}$ such that for each $k>K$ and for each $a_{i} \in A_{i}$ there is a $b_{i} \in B_{i}^{k}$ with $d_{A_{i}}\left(a_{i}, b_{i}\right)<\varepsilon,{ }^{6}$

[2] $\rho^{w}\left(\sigma_{i}^{k}, \sigma_{i}\right) \rightarrow 0$ as $k \rightarrow \infty$,

$$
\sum_{x \in P B R_{i}\left(\sigma^{k} \mid B_{i}^{k}\right)} \sigma_{i}^{k}(x) \rightarrow 1 \text { as } k \rightarrow \infty \quad\left(\text { where } \sigma^{k}=\left(\sigma_{i}^{k}\right)_{i \in N}\right){ }^{7}
$$

This definition has quite some appeal given our basic intuition that infinite games often serve as a proxy for large finite games, and that therefore strategic behavior should carry over from the finite setting to infinite games.

Despite this basic intuition, S\&S already noted that, even in games where players have a dominant action, lof perfection may fail to eliminate Nash equilibria that do

\footnotetext{
${ }^{6}$ This property can be expressed as " $d_{H}\left(B_{i}^{k}, A_{i}\right) \rightarrow 0$ as $k \rightarrow \infty$, where $d_{H}$ is Hausdorff distance".

${ }^{7}$ It is equivalent to $\rho^{s}\left(\sigma_{i}^{k}, B R_{i}\left(\sigma^{k} \mid B_{i}^{k}\right)\right) \rightarrow 0$ as $k \rightarrow \infty$.
} 
not use the dominant action (S\&S, Example 2.1). The following example illustrates these features of S\&S Examples 2.1 and 2.4 in a more straightforward game.

EXAMPLE 2. The action spaces are $A_{1}=\{T, B\}$ and $A_{2}=\{0,1,2, \ldots, \infty\}$, where all natural numbers are isolated points, whereas $\infty$ is the limit point of the sequence $0,1,2,3, \ldots$ The payoffs for the players are given by

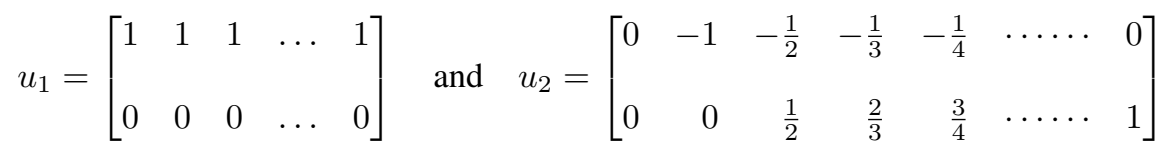

Claim. The action pair $\left(a_{1}, a_{2}\right)=(T, 0)$ is a lof perfect Nash equilibrium. However, $a_{2}=0$ is dominated by the (dominant) pure action $b_{2}=\infty$.

Proof. We show that $\left(a_{1}, a_{2}\right)=(T, 0)$ is lof perfect. Take $B_{1}^{k}=A_{1}=\{T, B\}$ and $B_{2}^{k}=\{0,1, \ldots, k\}$. Take a natural number $m>k$. Take $\sigma_{1}^{k}=\left(1-\frac{1}{m}\right) \delta(T)+$ $\frac{1}{m} \delta(B)$ and $\sigma_{2}^{k}=\left(1-\frac{1}{k}\right) \delta(0)+\frac{1}{k^{2}} \delta(1)+\ldots+\frac{1}{k^{2}} \delta(\infty)$. Clearly $\rho^{w}\left(\sigma_{1}^{k}, a_{1}\right) \rightarrow 0$ and $\rho^{w}\left(\sigma_{2}^{k}, a_{2}\right) \rightarrow 0$ as $k \rightarrow \infty$. Also [1] of Definition 4.2 is straightforward to check. We show that $\sigma_{1}^{k}$ and $\sigma_{2}^{k}$ satisfy condition [3] of Definition 4.2. It is trivial for player 1 , since $T$ is strictly dominates $B$ for player 1 .

We compute the payoff of a pure action $\ell \in B_{2}^{k}$ for player 2 against $\sigma_{1}^{k}$. If $\ell=0$, the payoff is evidently zero. Suppose $\ell \neq 0$. Then, since $\ell \leq k<m$, the payoff is

$$
-\left(1-\frac{1}{m}\right) \cdot \frac{1}{\ell}+\frac{1}{m} \cdot\left(1-\frac{1}{\ell}\right)=-\frac{1}{\ell}+\frac{1}{m \cdot \ell}+\frac{1}{m}-\frac{1}{m \cdot \ell}=\frac{1}{m}-\frac{1}{\ell}<0 .
$$

Hence, $\sigma_{2}^{k}$ puts weight $1-\frac{1}{k}$ on the pure best response within the set $B_{2}^{k}$, and $\left(a_{1}, a_{2}\right)=(T, 0)$ is lof perfect indeed.

In order to circumvent this drawback, $\mathrm{S} \& \mathrm{~S}$ introduced the notion of anchored perfect equilibrium. Let $F=\prod_{i \in N} F_{i} \subseteq A$ be finite.

Definition 2.3.3 (Simon and Stinchcombe) A strategy profile $\sigma=\left(\sigma_{i}\right)_{i \in N}$ is said to be anchored at $F$ if for every player $i \in N$ there is a sequence $\left(B_{i}^{k}\right)_{k=1}^{\infty}$ of finite subsets of $A_{i}$ with $F_{i} \subseteq B_{i}^{k}$ for every $i$ and $k$, and a sequence $\left(\sigma_{i}^{k}\right)_{k=1}^{\infty}$ of completely mixed strategies in $\Delta\left(B_{i}^{k}\right)$ such that for every $i \in N$

[1] for every $\varepsilon>0$ there is a $K \in \mathbf{N}$ such that for each $k>K$ and for each $a_{i} \in A_{i}$ there is a $b_{i} \in B_{i}^{k}$ with $d_{A_{i}}\left(a_{i}, b_{i}\right)<\varepsilon,{ }^{8}$

[2] $\rho^{w}\left(\sigma_{i}^{k}, \sigma_{i}\right) \rightarrow 0$ as $k \rightarrow \infty$,

[3] $\sum_{x \in P B R_{i}\left(\sigma^{k} \mid B_{i}^{k}\right)} \sigma_{i}^{k}(x) \rightarrow 1$ as $k \rightarrow \infty \quad\left(\right.$ where $\left.\sigma^{k}=\left(\sigma_{i}^{k}\right)_{i \in N}\right){ }^{9}$

\footnotetext{
${ }^{8}$ This property can be expressed as " $d_{H}\left(B_{i}^{k}, A_{i}\right) \rightarrow 0$ as $k \rightarrow \infty$, where $d_{H}$ is Hausdorff distance".

${ }^{9}$ It is equivalent to $\rho^{s}\left(\sigma_{i}^{k}, B R_{i}\left(\sigma^{k} \mid B_{i}^{k}\right)\right) \rightarrow 0$ as $k \rightarrow \infty$.
} 
A strategy profile $\sigma=\left(\sigma_{i}\right)_{i \in N}$ is an anchored perfect equilibrium if $\sigma$ is anchored at $F$ for every possible finite set $F=\prod_{i \in N} F_{i} \subseteq A$.

It is clear that the equilibrium $\left(a_{1}, a_{2}\right)=(T, 0)$ from Example 1 is not anchored perfect. So, as S\&S already noted, anchored perfection does move the finitistic approach closer to satisfying admissibility. However, because of the condition that we need to construct an approximating sequence for every possible finite subset $F$ of $A$, anchored perfection is in general quite difficult to verify (or to falsify for that matter). We propose the following modification of lof perfection.

Definition 2.3.4 A strategy profile $\sigma=\left(\sigma_{i}\right)_{i \in N}$ is a strongly global-limit-offinite (strongly glof) perfect equilibrium if in Definition 4.2 condition [3] is replaced by

[4] $\rho^{s}\left(\sigma_{i}^{k}, B R_{i}\left(\sigma^{k}\right)\right) \rightarrow 0$ as $k \rightarrow \infty$.

In comparison with condition [3], note that condition [4] is equivalent to

$$
\sum_{x \in B_{i}^{k} \cap P B R_{i}\left(\sigma^{k}\right)} \sigma_{i}^{k}(x) \rightarrow 1 \text { as } k \rightarrow \infty .
$$

The strategy profile $\sigma=\left(\sigma_{i}\right)_{i \in N}$ is a weakly glof perfect equilibrium if in Definition 4.2 condition [3] is replaced by

$$
\text { [5] } \rho^{w}\left(\sigma_{i}^{k}, B R_{i}\left(\sigma^{k}\right)\right) \rightarrow 0 \text { as } k \rightarrow \infty \text {. }
$$

As we demonstrate in the examples in this chapter, our modification of lof perfection is still comparatively straightforward to check, since it only involves construction of a single approximating sequence as in the definition of lof perfection. Moreover we show that our modification exclusively selects dominant strategies in case such strategies are available to a player. The main idea of the definition is that each player $i$ has to choose the probabilities with which he plays the actions in the finite approximation $B_{i}^{k}$ of his action space $A_{i}$ in such a way that the resulting probability distribution on $B_{i}^{k}$ is still close to his true best response in the action space $A_{i}$ itself given the strategies chosen by his opponents. This is in contrast to lof perfection in which attention is restricted only to the best responses within the finite approximation $B_{i}^{k}$ of $A_{i}$.

S\&S introduced the method of anchoring as a-partial-remedy to the failure of lof perfection to select limit admissible strategies. Nevertheless, anchoring still allows for the possibility that, no matter which set we selected a priori, best responses are not in the selected pure actions along the sequence. Our approach is more flexible in 
the sense that the inclusion of best responses is required to be contingent on the approximating sequence of strategy profiles we consider. Moreover, as already noted, checking anchored perfection requires us to construct an approximating sequence for every possible finite set $F \subseteq A$, while glof perfection only requires construction of a single approximating sequence.

Simon and Stinchcombe established existence of lof perfect and anchored perfect equilibrium. We show that weakly glof perfect equilibrium exists, and that every weakly glof perfect equilibrium is a Nash equilibrium. The rather lengthy proof is deferred to Appendix A.

Theorem 2.3.5 The set of weakly glof perfect equilibria of every strategic form game with compact action spaces and continuous payoff functions is a non-empty subset of the Nash equilibria that is closed with respect to the weak metric.

Since clearly every strongly glof perfect equilibrium is also weakly glof, both strong and weak glof perfection refines the set of Nash equilibria. Existence of strongly glof perfect equilibrium remains an open question.

\subsection{Admissibility}

In this section we analyze the relation between the various notions of perfection and admissibility. We show that weak perfection exclusively selects limit undominated strategies. We also show that this statement is a genuine generalization of the classical result for finite games that perfection implies undominatedness.

For the finitistic approach the connection to admissibility is much weaker. As said before in Example 2 of this chapter, Example 2.4 of S\&S already presents a lof perfect equilibrium that does not select the unique dominant strategy. We show that glof perfection performs better: for every game in which a player has dominant strategies, glof perfect equilibrium exclusively selects equilibria in which such a player plays one of his dominant strategies. However, the analogous result for undominated strategies again fails to hold. Example 3 presents a two-player game with a strongly glof perfect equilibrium in which both players play dominated strategies that are not limits of undominated strategies.

\section{Weak perfection and admissibility}

S\&S already showed that weakly perfect equilibrium is limit admissible. In this section we show the somewhat stronger claim that weakly perfect equilibrium strategies 
are limit undominated. We also show that this is the exact analogue of the classical result for finite games that perfect equilibrium strategies are undominated.

Theorem 2.4.1 Let $\sigma$ be a weakly perfect equilibrium. Then $\sigma_{i} \in L U_{i}$ for each $i$.

Proof. By Definition 4.1 and Theorem 3.2 of Billingsley [6], there exists a sequence of completely mixed strategy profiles $\sigma^{k}=\left(\sigma_{i}^{k}\right)_{i \in N}$ such that for every $i \in N$ we have $\rho^{w}\left(\sigma_{i}^{k}, \sigma_{i}\right) \rightarrow 0$ and $\rho^{w}\left(\sigma_{i}^{k}, B R_{i}\left(\sigma^{k}\right)\right) \rightarrow 0$ as $k \rightarrow \infty$.

So, for every $i \in N$, there is a sequence $\tau_{i}^{k} \in B R_{i}\left(\sigma^{k}\right)$ with $\rho^{w}\left(\sigma_{i}^{k}, \tau_{i}^{k}\right) \rightarrow 0$ as $k \rightarrow \infty$. Therefore, by the triangle inequality, $\rho^{w}\left(\tau_{i}^{k}, \sigma_{i}\right) \rightarrow 0$ as $k \rightarrow \infty$. Since $\tau_{i}^{k}$ is a best response against the completely mixed profile $\sigma^{k}$, by Lemma 2.9.2, $\tau_{i}^{k}$ is undominated. Therefore $\sigma_{i}$ is limit undominated.

At first glance Theorem 2.4.1 seems to be a compromise between the well-known result for finite normal form games that the strategies in a perfect equilibrium are undominated, and the transition to infinite compact action spaces. The following Proposition shows that this compromise is only seemingly, since for finite games limit undominated strategies are in fact undominated.

Proposition 2.4.2 For strategic form games with finite action spaces, the set $U_{i}$ is closed, so that $U_{i}=L U_{i}$ for such games.

Proof. To prove that the set of undominated strategies is closed, we show that the set of dominated strategies is open. Suppose $\sigma_{i}$ is dominated by $\tau_{i}$. We prove that there is an open set $V \ni \sigma_{i}$ such that every $\nu_{i} \in V$ is dominated as well. Define $\varepsilon=\frac{1}{2} \min \left\{\sigma_{i}(a) \mid a \in A_{i}\right.$ and $\left.\sigma_{i}(a)>0\right\}$. Since $A_{i}$ is a finite set, $\varepsilon>0$. Define

$$
V=\left\{\nu_{i} \in \Delta\left(A_{i}\right) \mid\left\|\nu_{i}-\sigma_{i}\right\|_{\infty}<\varepsilon\right\}
$$

Here, $\|\cdot\|_{\infty}$ denotes the maximum norm. Take $\nu_{i} \in V$. Write $\mu_{i}=2 \nu_{i}-\sigma_{i}$. We show that $\mu_{i} \in \Delta\left(A_{i}\right)$. Note that if $\sigma_{i}(a)=0$ then $\mu_{i}(a) \geq 0$. Now assume that $\sigma_{i}(a)>0$. Since $\left\|\nu_{i}-\sigma_{i}\right\|_{\infty}<\varepsilon$, we have

$$
\left|\nu_{i}(a)-\sigma_{i}(a)\right|<\varepsilon \leq \frac{1}{2} \sigma_{i}(a) .
$$

Consequently, $\mu_{i}(a)=2 \nu_{i}(a)-\sigma_{i}(a)=2 \nu_{i}(a)-2 \sigma_{i}(a)+\sigma_{i}(a)>0$. Because $\sum_{a \in A_{i}} \mu_{i}(a)=1$ clearly holds, we obtain $\mu_{i} \in \Delta\left(A_{i}\right)$.

We show that $\nu_{i}=\frac{1}{2} \sigma_{i}+\frac{1}{2} \mu_{i}$ is dominated by $\frac{1}{2} \tau_{i}+\frac{1}{2} \mu_{i}$. As $\sigma_{i}$ is dominated by $\tau_{i}$, there is a strategy profile $\rho_{-i} \in \Delta\left(A_{-i}\right)$ such that $u_{i}\left(\rho_{-i}, \tau_{i}\right)>u_{i}\left(\rho_{-i}, \sigma_{i}\right)$. 
Since $u_{i}$ is linear in each player's strategy we have

$$
\begin{aligned}
u_{i}\left(\rho_{-i}, \frac{1}{2} \tau_{i}+\frac{1}{2} \mu_{i}\right) & =\frac{1}{2} u_{i}\left(\rho_{-i}, \tau_{i}\right)+\frac{1}{2} u_{i}\left(\rho_{-i}, \mu_{i}\right) \\
& >\frac{1}{2} u_{i}\left(\rho_{-i}, \sigma_{i}\right)+\frac{1}{2} u_{i}\left(\rho_{-i}, \mu_{i}\right) \\
& =u_{i}\left(\rho_{-i}, \frac{1}{2} \sigma_{i}+\frac{1}{2} \mu_{i}\right) .
\end{aligned}
$$

Similarly we conclude for every $\sigma_{-i} \in \Delta\left(A_{-i}\right)$ that

$$
u_{i}\left(\sigma_{-i}, \frac{1}{2} \tau_{i}+\frac{1}{2} \mu_{i}\right) \geq u_{i}\left(\sigma_{-i}, \frac{1}{2} \sigma_{i}+\frac{1}{2} \mu_{i}\right) .
$$

Hence, $\nu_{i}=\frac{1}{2} \sigma_{i}+\frac{1}{2} \mu_{i}$ is dominated by $\frac{1}{2} \tau_{i}+\frac{1}{2} \mu_{i}$.

\section{Finitistic perfection and admissibility}

The relation between finitistic perfection and admissibility is much weaker than it is for weakly perfect equilibrium. Example 2 already shows that, even in games with dominant actions, lof perfection does not exclusively select equilibria in limit admissible strategies.

We show that our alternative definition of finitistic perfection, glof perfect equilibrium, does select only dominant strategies in case such strategies are available to a player. In an example we show that for games without dominant strategies also glof perfect equilibrium may use strategies that are not limit admissible.

Write $P D_{i}:=\left\{a_{i} \in A_{i} \mid \delta\left(a_{i}\right) \in D_{i}\right\}$. In Appendix B we show that $D_{i}$ is closed with respect to the weak metric in $\Delta\left(A_{i}\right)$, that $P D_{i}$ is closed in $A_{i}$, and that $D_{i}=\Delta\left(P D_{i}\right)$.

Theorem 2.4.3 Let $\sigma \in \Delta(A)$ be a weakly glof equilibrium. Suppose that $D_{i}$ is not empty. Then $\sigma_{i} \in D_{i}$.

Proof. Because $\sigma$ is a weakly glof equilibrium, for every player $i$ there is a sequence $\left(\sigma_{i}^{k}\right)_{k=1}^{\infty}$ such that $\rho^{w}\left(\sigma_{i}^{k}, \sigma_{i}\right) \rightarrow 0$ and $\rho^{w}\left(\sigma_{i}^{k}, B R_{i}\left(\sigma^{k}\right)\right) \rightarrow 0$ as $k \rightarrow \infty$. So, for every $k$ we can take $\tau_{i}^{k} \in B R_{i}\left(\sigma^{k}\right)$ such that $\rho^{w}\left(\sigma_{i}^{k}, \tau_{i}^{k}\right) \rightarrow 0$ as $k \rightarrow \infty$. Then, by the triangle inequality for $\rho^{w}$, also $\rho^{w}\left(\sigma_{i}, \tau_{i}^{k}\right) \rightarrow 0$ as $k \rightarrow \infty$.

Suppose that $D_{i}$ is not empty. Then, by Proposition 2.9.4, $P D_{i}$ is not empty either. Let $U \supset P D_{i}$ be open. First we show that $\tau_{i}^{k}(U)=1$ for sufficiently large $k$. When $U=A_{i}$, this is evident. Suppose $U \neq A_{i}$. Take $a_{i} \in A_{i} \backslash U$ and $b_{i} \in P D_{i}$. By Lemma 2.9.1, there is an $a_{-i} \in A_{-i}$ such that

$$
u_{i}\left(b_{i}, a_{-i}\right)>u_{i}\left(a_{i}, a_{-i}\right) .
$$


Since $u_{i}$ is continuous, there are open sets $V\left(a_{i}\right) \ni a_{i}$ and $W\left(a_{-i}\right) \ni a_{-i}$ such that for every $x_{i} \in V\left(a_{i}\right)$ and $x_{-i} \in W\left(a_{-i}\right)$ we have

$$
u_{i}\left(b_{i}, x_{-i}\right)>u_{i}\left(x_{i}, x_{-i}\right) .
$$

Take $x_{i} \in V\left(a_{i}\right)$. Since $b_{i}$ is a dominant strategy, we have

$$
u_{i}\left(b_{i}, y_{-i}\right) \geq u_{i}\left(x_{i}, y_{-i}\right)
$$

for every $y_{-i} \in A_{-i}$. Further, define $B_{-i}^{k}=\prod_{j \neq i} B_{j}^{k}$. Then $B_{-i}^{k} \cap W\left(a_{-i}\right) \neq \emptyset$ for large $k$ by Definition 4.3. So, since $\sigma_{-i}^{k}$ is a completely mixed strategy in $\Delta\left(B_{-i}^{k}\right)$, we know that

$$
u_{i}\left(b_{i}, \sigma_{-i}^{k}\right)>u_{i}\left(x_{i}, \sigma_{-i}^{k}\right)
$$

for large $k$. Hence, for large $k, x_{i}$ is not a best response to $\sigma^{k}$. We conclude for every $a_{i} \in A_{i} \backslash U$ that

$$
V\left(a_{i}\right) \cap P B R_{i}\left(\sigma^{k}\right)=\emptyset
$$

for large $k$. Now note that $A_{i} \backslash U$ is compact, since it is a closed subset of a compact set. Therefore, there are actions $a_{i}^{1}, \ldots, a_{i}^{p}$ in $A_{i} \backslash U$ such that $A_{i} \backslash U$ is a subset of $\bigcup_{r=1}^{p} V\left(a_{i}^{r}\right)$. Moreover,

$$
\operatorname{PBR}_{i}\left(\sigma^{k}\right) \cap \bigcup_{j=1}^{p} V\left(a_{i}^{j}\right)=\emptyset
$$

for large $k$. Consequently $P B R_{i}\left(\sigma^{k}\right) \subseteq U$ for large $k$. But $\tau_{i}^{k} \in B R_{i}\left(\sigma^{k}\right)$, so that $\tau_{i}^{k}\left(P B R_{i}\left(\sigma^{k}\right)\right)=1$ for all $k$. Hence, $\tau_{i}^{k}(U)=1$ for large $k$, as claimed.

We show that $\sigma_{i} \in D_{i}$. Take $\varepsilon>0$. Since $\rho^{w}\left(\sigma_{i}, \tau_{i}^{k}\right) \rightarrow 0$ as $k \rightarrow \infty$,

$$
\tau_{i}^{k}(U)-\varepsilon \leq \sigma_{i}\left(U^{\varepsilon}\right)
$$

for large $k$. Because $\tau_{i}^{k}(U)=1$ for large $k$, we get $1-\varepsilon \leq \sigma_{i}\left(U^{\varepsilon}\right)$. Since $\varepsilon>0$ and $U \supseteq P D_{i}$ were chosen arbitrarily, and since $P D_{i}$ is a closed set, we can conclude that $\sigma_{i}\left(P D_{i}\right)=1$. Hence, $\sigma_{i} \in \Delta\left(P D_{i}\right)=D_{i}$.

Theorem 2.4.3 shows that in games where players have dominant strategies, weak glof perfection can only select those equilibria in which dominant strategies are played. However, the next example shows that, in games where players do not have dominant strategies, even strong glof perfection may fail to exclusively select equilibria in limit admissible strategies.

EXAMPLE 3. The action spaces are

$$
A_{1}=A_{2}=\{-\infty, \ldots,-3,-2,-1,1,2,3, \ldots, \infty\},
$$


in which each integer is an isolated point, whereas $-\infty$ is the limit of the sequence $-1,-2, \ldots$ and $\infty$ is the limit of the sequence $1,2, \ldots$. The topological structure is shown in the picture below.

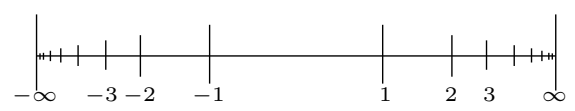

The payoff functions $u_{1}$ and $u_{2}$ are symmetric and $u_{1}$ is given in the table below.

\begin{tabular}{c|ccccc|ccccc|}
\hline$u_{1}$ & $-\infty$ & $\ldots$ & -3 & -2 & -1 & 1 & 2 & 3 & $\ldots$ & $\infty$ \\
\hline$\infty$ & 0 & $\ldots$ & 0 & 0 & 0 & 0 & 0 & 0 & $\ldots$ & 0 \\
$\vdots$ & $\vdots$ & $\ddots$ & $\vdots$ & $\vdots$ & $\vdots$ & $\vdots$ & $\vdots$ & $\vdots$ &. & $\vdots$ \\
-3 & 0 & $\ldots$ & $\frac{1}{3}$ & 0 & 0 & 0 & 0 & $\frac{1}{3}$ & $\ldots$ & 0 \\
-2 & 0 & $\ldots$ & 0 & $\frac{1}{2}$ & 0 & 0 & $\frac{1}{2}$ & 0 & $\ldots$ & 0 \\
-1 & 0 & $\ldots$ & 0 & 0 & 1 & 1 & 0 & 0 & $\ldots$ & 0 \\
1 & 0 & $\ldots$ & 0 & 0 & 0 & 1 & 0 & 0 & $\ldots$ & 0 \\
2 & 0 & $\ldots$ & 0 & 0 & 0 & 0 & $\frac{1}{2}$ & 0 & $\ldots$ & 0 \\
3 & 0 & $\ldots$ & 0 & 0 & 0 & 0 & 0 & $\frac{1}{3}$ & $\ldots$ & 0 \\
$\vdots$ & $\vdots$ &.$\cdot$ & $\vdots$ & $\vdots$ & $\vdots$ & $\vdots$ & $\vdots$ & $\vdots$ & $\ddots$ & $\vdots$ \\
$\infty$ & 0 & $\ldots$ & 0 & 0 & 0 & 0 & 0 & 0 & $\ldots$ & 0 \\
\hline
\end{tabular}

Player 1 is the row player, and player 2 is the column player.

Claim. The action pair $(\infty, \infty)$ is a strongly glof perfect equilibrium. However, $\infty$ is not limit admissible for either player.

Proof. For either player, $\infty$ is not limit admissible because $\infty$ is dominated by every action $k \in \mathbb{Z}$, and every action $k \in \mathbf{N}$ is dominated by action $-k$.

Now, we prove that $\sigma=(\infty, \infty)$ is a strongly glof perfect equilibrium. For every player $i=1,2$ and $k \in \mathbb{N}$, let $B_{i}^{k}=\{-k, \ldots,-1,1, \ldots, k+1\}$ and let $\sigma_{i}^{k}$ be the completely mixed strategy on $B_{i}^{k}$ which assigns probability $\frac{1}{(k+2)^{2}}$ to every action in $B_{i}^{k}$ except action $k+1$ and assigns probability $\left(1-\frac{2 k}{(k+2)^{2}}\right)$ to action $k+1$. It is clear 
that $\left(B_{i}^{k}\right)_{k=1}^{\infty}$ satisfies the first condition of Definition 4.4. Since $\rho^{w}\left(\sigma_{i}^{k}, \sigma_{i}\right) \rightarrow 0$ for $i=1,2$ as $k \rightarrow \infty$, the second condition also holds. Using the payoff table, one can verify that strategies $(-k-1)$ and $(k+1)$ are the best responses for player 1 against $\sigma_{2}^{k}$ in $A_{1}$. Due to symmetry, the same holds for player 2 . Thus,

$$
\rho^{s}\left(\sigma_{i}^{k}, B R_{i}\left(\sigma^{k}\right)\right)=1-\left(1-\frac{2 k}{(k+2)^{2}}\right)=\frac{2 k}{(k+2)^{2}},
$$

which tends to zero as $k \rightarrow \infty$. Hence, condition [4] of Definition 4.4 is valid as well, and $\sigma$ is a strongly glof perfect equilibrium.

\subsection{Relations between definitions of perfect equilibrium}

Simon and Stinchcombe [42] conjectured (on page 1433) that there are strongly perfect equilibria that are not anchored perfect. We provide a concrete example of a completely mixed, and therefore surely strongly perfect, equilibrium that is neither lof perfect nor glof perfect (and therefore also not anchored perfect). Thus, the finitistic approach to perfection does not have a straightforward logical relation to the trembling hand approach.

EXAMPLE 4. The action spaces are $A_{1}=A_{2}=\{1,2,3, \ldots, \infty, \infty+1\}$, where all natural numbers and $\infty+1$ are isolated points, whereas $\infty$ is the limit of the sequence $1,2, \ldots$ The topology is shown in the picture below:

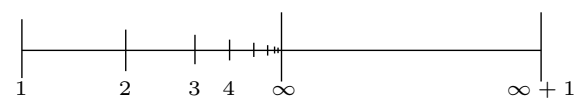

The payoff functions $u_{1}$ and $u_{2}$ are symmetric and $u_{1}$ is given in the table below.

\begin{tabular}{|c|rrrrrrr|}
\hline$u_{1}$ & 1 & 2 & 3 & 4 & $\ldots$ & $\infty$ & $\infty+1$ \\
\hline 1 & $\frac{4}{8^{2}}$ & $\frac{-1}{8}$ & 0 & 0 & $\ldots$ & 0 & 0 \\
2 & $\frac{4}{16^{2}}$ & 0 & $\frac{-1}{16}$ & 0 & $\ldots$ & 0 & 0 \\
3 & $\frac{4}{32^{2}}$ & 0 & 0 & $\frac{-1}{32}$ & $\ldots$ & 0 & 0 \\
4 & $\frac{4}{64^{2}}$ & 0 & 0 & 0 & $\ldots$ & 0 & 0 \\
$\vdots$ & $\vdots$ & $\vdots$ & $\vdots$ & $\vdots$ & $\ddots$ & $\vdots$ & $\vdots$ \\
$\infty$ & 0 & 0 & 0 & 0 & $\ldots$ & 0 & 0
\end{tabular}


Define the strategies $\sigma_{1}$ and $\sigma_{2}$ by

$$
\sigma_{1}=\sigma_{2}=\frac{1}{4} \cdot \delta(1)+\frac{1}{8} \cdot \delta(2)+\frac{1}{16} \cdot \delta(3)+\ldots+\frac{1}{4} \cdot \delta(\infty)+\frac{1}{4} \cdot \delta(\infty+1) .
$$

Claim. The strategy profile $\left(\sigma_{1}, \sigma_{2}\right)$ is a completely mixed, and hence strongly perfect equilibrium. However, it is neither lof perfect nor weakly glof perfect. This verifies the conjecture of S\&S [42]. ${ }^{10}$

Proof. It is clear that $\left(\sigma_{1}, \sigma_{2}\right)$ is a completely mixed Nash equilibrium. Hence, it is a strongly perfect equilibrium.

We show that the completely mixed equilibrium is not lof perfect. Suppose by way of contradiction that $\left(\sigma_{1}, \sigma_{2}\right)$ is lof perfect. For every player $i$, take a sequence $\left(B_{i}^{k}\right)_{k=1}^{\infty}$ of finite subsets of $A_{i}$ and a sequence of strategies $\left(\sigma_{i}^{k}\right)_{k=1}^{\infty}$ that satisfy the conditions in Definition 4.2. Let $m_{k}$ and $n_{k}$ be the largest positive integers in the sets $B_{1}^{k}$ and $B_{2}^{k}$, respectively. Since $\infty+1$ is an isolated point of the action sets, for large $k$ each $B_{i}^{k}$ includes $\infty+1$. Moreover, for large $k, \infty+1$ must be a best response for player 1 against $\sigma_{2}^{k}$ among $B_{1}^{k}$, because $\sigma_{1}$ places positive probability on $\infty+1$. As strategy $\infty+1$ has payoff zero for player 1 , for large $k$ player 1 cannot have an action in $B_{1}^{k}$ which gives him a positive payoff against $\sigma_{2}^{k}$. Since player 1 would get a positive payoff for every action $m \geq n_{k}$, we have $m_{k}<n_{k}$. Then also $n_{k}<m_{k}$ by symmetry, which is a contradiction. By similar arguments the completely mixed equilibrium is not weakly glof either.

We continue with a discussion of the relations between the different approaches to finitistic perfect equilibrium.

Proposition 2.5.1 Every strongly glof perfect equilibrium is lof perfect.

Proof. Suppose that $\sigma=\left(\sigma_{i}\right)_{i \in N}$ is a strongly glof perfect equilibrium. Then, for every $i \in N$, there is a sequence $\left(B_{i}^{k}\right)_{k=1}^{\infty}$ of finite subsets of $A_{i}$ and a sequence of strategies $\left(\sigma_{i}^{k}\right)_{k=1}^{\infty}$ that satisfy the conditions in Definition 4.4. We show that they also satisfy condition [3] of Definition 4.2. Since for every $i$, we have $\rho^{s}\left(\sigma_{i}^{k}, B R_{i}\left(\sigma^{k}\right)\right) \rightarrow 0$ as $k \rightarrow \infty$, there exists a sequence $\left(\mu_{i}^{k}\right)_{k=1}^{\infty}$ such that $\mu_{i}^{k} \in B R_{i}\left(\sigma^{k}\right)$ for every $k \in \mathbf{N}$ and $\rho^{s}\left(\sigma_{i}^{k}, \mu_{i}^{k}\right) \rightarrow 0$ as $k \rightarrow \infty$. As $u_{i}$ is a continuous function, $P B R_{i}\left(\sigma^{k}\right)$ is a closed, hence Borel, subset of the action space $A_{i}$. Moreover, since best responses put weight 1 on pure best responses,

$$
\rho^{s}\left(\sigma_{i}^{k}, \mu_{i}^{k}\right) \geq\left|\sigma_{i}^{k}\left(P B R_{i}\left(\sigma^{k}\right)\right)-\mu_{i}^{k}\left(P B R_{i}\left(\sigma^{k}\right)\right)\right|=\left|\sigma_{i}^{k}\left(P B R_{i}\left(\sigma^{k}\right)\right)-1\right|
$$

\footnotetext{
${ }^{10}$ Their conjecture only concerns anchored perfect equilibrium. We show the stronger claim that the completely mixed equilibrium is even not a lof perfect equilibrium.
} 
for every $i$ and $k$. So, $\sigma_{i}^{k}\left(\operatorname{PBR}_{i}\left(\sigma^{k}\right)\right) \rightarrow 1$ as $k \rightarrow \infty$. This implies that for large $k$, player $i$ has a pure best response to $\sigma^{k}$ which lies in $B_{i}^{k}$. Consequently, $\sigma_{i}^{k}\left(P B R_{i}\left(\sigma^{k} \mid B_{i}^{k}\right)\right) \rightarrow 1$ as $k \rightarrow \infty$, and $\sigma$ is a lof perfect equilibrium.

Theorem 2.4.3 shows that the lof perfect equilibrium in Example 1 is not weakly glof perfect (and hence not strongly glof perfect either). Next we present an example of a weakly glof perfect equilibrium that is not lof perfect. Consequently, lof perfection and weak glof perfection are not comparable.

EXAMPLE 5. In this game the action spaces are

$$
A_{1}=A_{2}=\{-1,-2,-3, \ldots, 0, \ldots, 3,2,1\}
$$

where all integers except 0 are isolated points, whereas 0 is the limit point of the sequences $-1,-2, \ldots$ and $1,2, \ldots$ The topological structure is shown in the picture below.

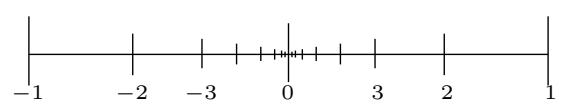

The topology can be metrized by the following metric. For every $k, l \in A_{i}$,

$$
d(k, l)= \begin{cases}0 & \text { if } k=l=0 \\ \left|\frac{1}{l}\right| & \text { if } k=0 \text { and } l \neq 0 \\ \left|\frac{1}{k}\right| & \text { if } k \neq 0 \text { and } l=0 \\ \left|\frac{1}{k}-\frac{1}{l}\right| & \text { if } k \neq 0 \text { and } l \neq 0\end{cases}
$$

The payoff functions $u_{1}$ and $u_{2}$ are symmetric and $u_{1}$ is given in the table below. 


\begin{tabular}{|c|cccc|c|cccc|}
\hline$u_{1}$ & -1 & -2 & -3 & $\cdots$ & 0 & $\cdots$ & +3 & +2 & +1 \\
\hline-1 & 1 & $\frac{1}{2}$ & $\frac{1}{3}$ & $\cdots$ & 0 & $\cdots$ & 0 & 0 & 1 \\
-2 & 0 & 0 & 0 & $\cdots$ & 0 & $\cdots$ & 0 & $\frac{1}{2}$ & 0 \\
-3 & 0 & 0 & 0 & $\cdots$ & 0 & $\cdots$ & $\frac{1}{3}$ & 0 & 0 \\
$\vdots$ & $\vdots$ & $\vdots$ & $\vdots$ & $\ddots$ & $\vdots$ &. & $\vdots$ & $\vdots$ & $\vdots$ \\
0 & 0 & 0 & 0 & $\cdots$ & 0 & $\cdots$ & 0 & 0 & 0 \\
\hline$\vdots$ & $\vdots$ & $\vdots$ & $\vdots$ &.$\cdot$ & $\vdots$ & $\ddots$ & $\vdots$ & $\vdots$ & $\vdots$ \\
+3 & 0 & 0 & 0 & $\cdots$ & 0 & $\cdots$ & 0 & 0 & 0 \\
+2 & 0 & 0 & 0 & $\cdots$ & 0 & $\cdots$ & 0 & 0 & 0 \\
+1 & 0 & 0 & 0 & $\cdots$ & 0 & $\cdots$ & 0 & 0 & 0 \\
\hline
\end{tabular}

Claim. The Nash equilibrium $\left(\sigma_{1}, \sigma_{2}\right)=(0,0)$ is weakly glof perfect. However, $\left(\sigma_{1}, \sigma_{2}\right)$ is not lof perfect, and therefore by Proposition 2.5.1 not strongly glof perfect either.

Proof. We show that $\left(\sigma_{1}, \sigma_{2}\right)$ is a weakly glof perfect. For every $k \in \mathbf{N}$, define $\varepsilon_{k}=\frac{1}{k(k+3)}$. Moreover, for $k \in \mathbf{N}$ and $i=1,2$, write

$$
B_{i}^{k}=\{-1,-2, \ldots,-k\} \bigcup\{k, \ldots, 2,1\} .
$$

Let $\sigma_{i}^{k}$ be the completely mixed strategy on $B_{i}^{k}$ that assigns probability $\varepsilon_{k}$ to every action in $B_{i}^{k}$ except action $k$, to which it assigns probability $1-(2 k-1) \varepsilon_{k}$. Note that due to the choice of $\varepsilon_{k}$, we have $1-(2 k-1) \varepsilon_{k}>\varepsilon_{k}>0$. It is clear that conditions [1] and [2] of Definition 4.4 are satisfied. It remains to verify that condition [5] holds as well. According to the payoff table, player 1's pure best response to $\sigma_{2}^{k}$ is either -1 or $-k$. Since for every $k \geq 2$ we have

$$
\begin{aligned}
u_{1}\left(\delta(-1), \sigma_{2}^{k}\right) & =\varepsilon_{k}\left(1+\frac{1}{2}+\frac{1}{3}+\ldots+\frac{1}{k}+1\right) \\
\leq & \varepsilon_{k}(k+1) \\
& <\left(1-(2 k-1) \varepsilon_{k}\right) \frac{1}{k} \\
& =u_{1}\left(\delta(-k), \sigma_{2}^{k}\right)
\end{aligned}
$$


player 1's unique best response against $\sigma_{2}^{k}$ is $-k$. We prove that

$$
\rho^{w}\left(\sigma_{1}^{k}, B R_{1}\left(\sigma^{k}\right)\right)=\rho^{w}\left(\sigma_{1}^{k}, \delta(-k)\right) \leq \frac{2}{k} .
$$

Let $\varepsilon>\frac{2}{k}=d(-k, k)$. It is easy to see that $(2 k-1) \varepsilon_{k} \leq \varepsilon$. Take an arbitrary set $B \subseteq A_{1}$.

A. We show that $\delta(-k)(B) \leq \sigma_{1}^{k}\left(B^{\varepsilon}\right)+\varepsilon$. If $-k \notin B$. Then

$$
\delta(-k)(B)=0 \leq \sigma_{1}^{k}\left(B^{\varepsilon}\right)+\varepsilon .
$$

If $-k \in B$. Then $k \in B^{\varepsilon}$. So

$$
\delta(-k)(B)=1 \leq\left(1-(2 k-1) \varepsilon_{k}\right)+\varepsilon=\sigma_{1}^{k}(k)+\varepsilon \leq \sigma_{1}^{k}\left(B^{\varepsilon}\right)+\varepsilon .
$$

B. We show that $\sigma_{1}^{k}(B) \leq \delta(-k)\left(B^{\varepsilon}\right)+\varepsilon$. If $-k \in B$. Then

$$
\sigma_{1}^{k}(B) \leq 1<1+\varepsilon=\delta(-k)\left(B^{\varepsilon}\right)+\varepsilon
$$

If $-k \notin B$ and $k \notin B$. Then

$$
\sigma_{1}^{k}(B) \leq(2 k-1) \varepsilon_{k} \leq \varepsilon \leq \delta(-k)\left(B^{\varepsilon}\right)+\varepsilon .
$$

If $-k \notin B$ and $k \in B$. Then $-k \in B^{\varepsilon}$. So

$$
\sigma_{1}^{k}(B) \leq 1+\varepsilon=\delta(-k)\left(B^{\varepsilon}\right)+\varepsilon
$$

So, by $\mathbf{A}, \mathbf{B}$, and the choice of $\varepsilon, \rho^{w}\left(\sigma_{1}^{k}, \delta(-k)\right) \leq \frac{2}{k}$. Since the payoff functions are symmetric this argument is also valid for the second player. Therefore, condition [5] of Definition 4.4 holds and $\sigma$ is a weakly glof perfect equilibrium as claimed ${ }^{11}$.

We show that $(0,0)$ is not lof perfect. Suppose the opposite. Then by Definition 4.2 for each $i=1,2$ there is non-empty finite subset $B_{i}$ of $A_{i}$ and a completely mixed strategy $\sigma_{i}$ in $\Delta\left(B_{i}\right)$ such that

$$
\sigma_{i}\left(P B R_{i}\left(\sigma \mid B_{i}\right)\right) \geq \frac{2}{3}
$$

where $\sigma=\left(\sigma_{1}, \sigma_{2}\right)$. Since -1 is an isolated point, w.l.o.g. $-1 \in B_{i}$ for each $i=1,2$. Furthermore, $P B R_{i}\left(\sigma \mid B_{i}\right)$ is a subset of $\{-1,-2, \ldots\}$. Define

$$
M_{i}=-\min \left\{x \mid x \in \operatorname{PBR}_{i}\left(\sigma \mid B_{i}\right)\right\} \quad \text { for } i=1,2 .
$$

\footnotetext{
${ }^{11}$ Note that strategy $\sigma_{i}^{k}$ puts almost all weight on pure action $+k$, which is in fact a worst pure action in terms of payoffs.
} 
Assume w.l.o.g. that $M_{1} \geq M_{2}$. Because $-M_{1} \in P B R_{1}\left(\sigma \mid B_{1}\right)$ and $-1 \in B_{1}$ we have

$$
u_{1}\left(\delta(-1), \sigma_{2}\right) \leq u_{1}\left(\delta\left(-M_{1}\right), \sigma_{2}\right)
$$

Thus, according to the payoff table,

$$
\frac{2}{3} \cdot \frac{1}{M_{2}} \leq u_{1}\left(\delta(-1), \sigma_{2}\right) \leq u_{1}\left(\delta\left(-M_{1}\right), \sigma_{2}\right) \leq \frac{1}{3} \cdot \frac{1}{M_{1}}
$$

Rewriting yields $\frac{M_{2}}{2} \geq M_{1}$. Consequently $\frac{M_{2}}{2} \geq M_{2}$. Contradiction.

Finally, notice that in the pure lof perfect equilibrium of Example 1, players 2 plays an action that is not just not limit admissible, it is even dominated by a unique dominant action. It follows from Theorem 2.4.3 that this equilibrium is not weakly glof.

\subsection{Two players}

For finite strategic form games it is well-known that perfection implies undominatedness. A similarly well-known theorem of van Damme (see for example van Damme [43], Theorem 3.2.2) states that for bimatrix games the converse implication also holds.

Theorem 2.6.1 Let $(p, q)$ be a strategy pair of the bimatrix game $(A, B)$. Equivalent are

[1] The strategy pair $(p, q)$ is a perfect equilibrium.

[2] The strategy pair $(p, q)$ is a Nash equilibrium and both $p$ and $q$ are undominated strategies.

Theorem 2.4.1 and Proposition 2.4.2 show that, in the appropriately adjusted sense, the implication from [1] to [2] is also valid in the setting with infinite compact action spaces. A natural guess would be that the appropriately adjusted converse would also be true for two-player infinite games. However, the following example shows that the converse implication from [2] to [1] no longer holds for games with infinite action spaces. (In their Example 2 Méndez et al. [27] address the same issue in a game with interval action spaces.)

EXAMPLE 6. The action spaces are $A_{1}=\{T, B\}$ and $A_{2}=\{1,2,3,4, \ldots, \infty\}$, where all natural numbers are isolated points, whereas $\infty$ is the limit point of the sequence $1,2,3, \ldots$ The payoffs for the players are given by 


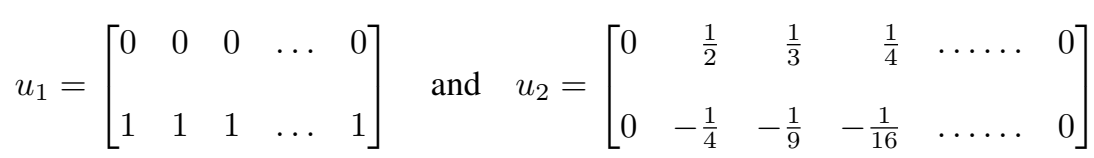

Claim. The action pair $(B, 1)$ is a Nash equilibrium in undominated strategies, yet $(B, 1)$ is not a weakly perfect equilibrium.

Proof. Clearly $(B, 1)$ is a Nash equilibrium and actions $B$ and 1 are undominated in $A_{1}$ and $A_{2}$ respectively.

We prove that the action pair $\sigma=(B, 1)$ is not a weakly perfect equilibrium. Take any completely mixed strategy $\tau_{1}=(p, 1-p)$ of player 1 . Take a natural number $k>\frac{1-p}{p}$. Then $\frac{1-p}{k}<p$, so that

$$
\begin{aligned}
u_{2}\left(\tau_{1}, \delta(k)\right) & =p \cdot \frac{1}{k}-(1-p) \cdot \frac{1}{k^{2}} \\
& =\frac{1}{k} \cdot\left[p-\frac{1-p}{k}\right] \\
& >0 .
\end{aligned}
$$

Since $u_{2}\left(\tau_{1}, \delta(1)\right)=0$, this implies that $\delta(1)$ is not a best response for player 2 against any completely mixed strategy of player 1 . Hence, $\sigma=(B, 1)$ is not a weakly perfect equilibrium.

Note that the above example is in a very strong sense minimal. The action space $A_{2}$ is in a very natural way the "smallest" example of a compact set that is not finite. And also $A_{1}$ is minimal in the sense that it is the smallest example of an action space in which player 1 actually has a genuine choice to make.

\subsection{Invariance}

Another remarkable feature of the game in Example 6 is that $(B, \infty)$ is a strongly (and hence also weakly) perfect equilibrium. This is surprising in the sense that $\infty$ is payoff equivalent to 1 for player 2 . Thus, both strong and weak perfection violate invariance in this setting, while perfect equilibrium satisfies invariance in the setting of finite strategic form games (see for example Mertens [30] and Vermeulen and Jansen [44]).

The underlying cause of this seeming breakdown of invariance is that in the finite case the space of actions is, from a topological perspective, a very simple object. All actions are isolated points, and hence there is a homeomorphism that transforms one 
into the other while the induced transformation on payoffs equals the identity in the case of payoff equivalence.

However, in the infinite case two actions giving the same payoff might be totally different from a topological perspective. Therefore, only conditional on having a homeomorphism that preserves payoffs, we can conclude that strong and weak perfection are invariant too. This result is stated in the following theorem.

Theorem 2.7.1 Both strongly and weakly perfect equilibrium are invariant under payoff preserving homeomorphic transformations of the action spaces.

Note that, for finite games, invariance with respect to payoff preserving homeomorphic transformations is in fact equivalent to invariance. Hence, the above Theorem implies that for finite strategic form games perfect equilibrium is invariant.

\subsection{Discussion}

We studied the relations between various generalizations of the basic notion of perfect equilibrium to the context of strategic form games with compact action spaces and continuous payoff functions. We showed the existence of weakly glof perfect equilibrium, and presented several examples of games with compact action spaces that clarify the relationships between admissibility, trembling hand perfect equilibrium, and finitistic perfect equilibrium.

Specifically, we showed in Example 6 that the equivalence between perfection and undominatedness for bimatrix games no longer holds for general two-person strategic form games with compact action spaces and continuous payoffs.

We also verified a conjecture of Simon and Stichcombe [42] with an example of a completely mixed Nash equilibrium that is not perfect in any of the finitistic definitions. Example 3 shows that finitistic perfection may easily fail to be limit admissible, a fact that was already observed by Simon and Stinchcombe for lof perfection. Example 4 shows that a completely mixed equilibrium need not be perfect in any of the finitistic approaches.

In Example 4 the completely mixed equilibrium is part of a component of Nash equilibria, one of which is still finitistically perfect. It remains an open question whether an isolated completely mixed equilibrium is automatically perfect in the finitistic approach. Another possible question for future research is the existence of strongly glof perfect equilibrium. 
Despite its appeal to basic intuition of the finitistic approach, our results (especially Examples 3 and 4) seem to imply a severe critique of the finitistic approach. The connection of the finitistic notions of perfection to admissibility and undominatedness seems hazy at best, and a cure for this — still somewhat surprising-failure of finitistic solutions on simple performance criteria such as undominatedness does not seem to be easily available. Still, as the anonymous referee pointed out, there may be an alternative interpretation for our results. Flipping our stance towards the finitistic approach-that finite games are "approximations" for the "true" infinite game we want to study - around, we could also adopt the view that all games people might ever encounter are finite. Under that view, infinite games function as "approximations" of large, but still finite, games. Infinite games in this view are only analytic tools, they are not the true game under consideration. Our results now show that this view, infinite games function as approximations of large finite games to facilitate computation of solutions, can no longer be maintained when we wish to apply perfect equilibrium as the central solution concept ${ }^{12}$.

\subsection{Appendix}

\section{Proof of Theorem 2.3.5}

The proof of Theorem 2.3.5 is based on the fixed point Theorem of Kakutani. Since $A_{i}$ is compact and metric, we know that it is totally bounded. Fix $k \in \mathbf{N}$. For every $i$, construct a finite subset $B_{i}^{k}$ of $A_{i}$ such that for every $a \in A_{i}$ there is a $b \in B_{i}^{k}$ with $d_{A_{i}}(a, b) \leq \frac{1}{k}$. Write $n_{i k}=\left|B_{i}^{k}\right|$, and $n_{k}=\max \left\{n_{i k}\right\}$. Take $\varepsilon \in\left(0, \frac{1}{n_{k}} \cdot \frac{1}{k}\right)$. W.1.o.g. we can assume that $n_{i k} \geq 2$. For every $i$, define

$$
\Delta_{i}^{k}(\varepsilon)=\left\{\nu_{i} \in \Delta\left(B_{i}^{k}\right) \mid \nu_{i}(b) \geq \varepsilon \text { for every } b \in B_{i}^{k}\right\}
$$

and $\Delta^{k}(\varepsilon)=\prod_{i \in N} \Delta_{i}^{k}(\varepsilon)$. Define the correspondence $A B R_{i}^{k}: \Delta^{k}(\varepsilon) \rightarrow \Delta_{i}^{k}(\varepsilon)$ by

$$
A B R_{i}^{k}(\sigma)=\left\{\mu_{i} \in \Delta_{i}^{k}(\varepsilon) \mid \rho^{w}\left(\mu_{i}, B R_{i}(\sigma)\right) \leq \frac{2}{k}\right\}
$$

\footnotetext{
${ }^{12}$ Perhaps a bit bold to put this chapter on par with the development of quantum physics, but exactly this phenomenon occurred when physicists tried to explain Kirchoff's laws for the solar emission spectrum. Classical physics that treated light as a continuous wave could not explain the shape of the solar spectrum. This forced Planck in 1900 to propose a discretized model of light emission, where light was only allowed to be emitted in little blocks of fixed size, dubbed "quanta", which exactly reproduced the solar spectrum. Planck had made the first step in the development of quantum physics. Apparently, at least when it concerned light emission spectra, reality was undeniably discrete, no continuous model for light emission could explain the observations of the solar spectrum. Now, we are definitely aware of the fact that thus far the theory on infinite games has not come up with its own version of the solar emission spectrum. Still, as an analogy to clarify the view pointed out by the referee, we believe the story of the birth of quantum physics is unrivaled.
} 
for every $\sigma=\left(\sigma_{i}\right)_{i \in N} \in \Delta^{k}(\varepsilon) .{ }^{13}$ Furthermore, for every $\sigma \in \Delta(A)$, define the correspondence $A B R^{k}: \Delta^{k}(\varepsilon) \rightarrow \Delta^{k}(\varepsilon)$ by

$$
A B R^{k}(\sigma)=\prod_{i \in N} A B R_{i}^{k}(\sigma)
$$

We verify the conditions of Theorem 4.6.6.

I. Clearly $\Delta^{k}(\varepsilon)$ is compact and convex. Further, since $\varepsilon \leq \frac{1}{n_{k}} \leq \frac{1}{n_{i k}}$, the set $\Delta^{k}(\varepsilon)$ is not empty.

II. We verify that $A B R^{k}(\sigma)$ satisfies the conditions of the Theorem of Kakutani.

II.1. We show for every $i \in N$ that $A B R_{i}^{k}(\sigma)$ is non-empty. Take $a \in$ $\operatorname{PBR}_{i}(\sigma)$. Then, there is a $b \in B_{i}^{k}$ such that $d_{A_{i}}(a, b) \leq \frac{1}{k}$. Hence, $\rho^{w}(\delta(a), \delta(b)) \leq$ $\frac{1}{k}$. Now, for $x \in B_{i}^{k}$ define

$$
\mu_{i}(x)= \begin{cases}\varepsilon & \text { if } x \neq b \\ 1-\left(n_{i k}-1\right) \cdot \varepsilon & \text { if } x=b .\end{cases}
$$

Then, $\mu_{i} \in \Delta_{i}^{k}(\varepsilon)$. Moreover,

$$
\rho^{w}\left(\mu_{i}, \delta(b)\right) \leq\left\|\mu_{i}-\delta(b)\right\|_{\infty}=\left(n_{i k}-1\right) \cdot \varepsilon \leq\left(n_{i k}-1\right) \cdot \frac{1}{n_{k}} \cdot \frac{1}{k} \leq \frac{1}{k} .
$$

Then, by the triangle inequality we have

$$
\rho^{w}\left(\mu_{i}, \delta(a)\right) \leq \frac{1}{k}+\frac{1}{k}=\frac{2}{k} .
$$

Thus, $\rho^{w}\left(\mu_{i}, B R_{i}(\sigma)\right) \leq \frac{2}{k}$, which implies $\mu_{i} \in A B R_{i}^{k}(\sigma)$. Hence, $A B R^{k}(\sigma)$ is non-empty.

II.2. Clearly, for every $i, A B R_{i}^{k}(\sigma)$ is closed and therefore $A B R^{k}(\sigma)$ is closed as well.

II.3. We prove that $A B R_{i}^{k}(\sigma)$ is convex for every $i$. Take strategies $\mu, \nu \in$ $A B R_{i}^{k}(\sigma)$. We prove that $c \mu+(1-c) \nu \in A B R_{i}^{k}(\sigma)$ for every $c \in[0,1]$. Since $\mu, \nu \in A B R_{i}^{k}(\sigma)$, we have $\rho^{w}\left(\mu, B R_{i}(\sigma)\right) \leq \frac{2}{k}$ and $\rho^{w}\left(\nu, B R_{i}(\sigma)\right) \leq \frac{2}{k}$. So there are strategies $\mu^{\prime}, \nu^{\prime} \in B R_{i}(\sigma)$ such that

$$
\rho^{w}\left(\mu, \mu^{\prime}\right) \leq \frac{2}{k} \quad \text { and } \quad \rho^{w}\left(\nu, \nu^{\prime}\right) \leq \frac{2}{k} .
$$

\footnotetext{
${ }^{13} A B R$ stands for approximate best response.
} 
Take an arbitrary $\delta>\frac{2}{k}$. Then, for every non-empty measurable set $A \subseteq A_{i}$, we have

$$
\begin{aligned}
& \mu(A) \leq \mu^{\prime}\left(A^{\delta}\right)+\delta \\
& \mu^{\prime}(A) \leq \mu\left(A^{\delta}\right)+\delta
\end{aligned}
$$

and

$$
\begin{gathered}
\nu(A) \leq \nu^{\prime}\left(A^{\delta}\right)+\delta \\
\nu^{\prime}(A) \leq \nu\left(A^{\delta}\right)+\delta .
\end{gathered}
$$

Then,

$$
\begin{aligned}
& c \mu(A)+(1-c) \nu(A) \leq c \mu^{\prime}\left(A^{\delta}\right)+(1-c) \nu^{\prime}\left(A^{\delta}\right)+\delta \\
& c \mu^{\prime}(A)+(1-c) \nu^{\prime}(A) \leq c \mu\left(A^{\delta}\right)+(1-c) \nu\left(A^{\delta}\right)+\delta .
\end{aligned}
$$

Hence,

$$
\rho^{w}\left(c \mu+(1-c) \nu, c \mu^{\prime}+(1-c) \nu^{\prime}\right) \leq \delta
$$

which by the choice of $\delta$ implies

$$
\rho^{w}\left(c \mu+(1-c) \nu, c \mu^{\prime}+(1-c) \nu^{\prime}\right) \leq \frac{2}{k}
$$

Since $B R_{i}(\sigma)$ is a convex set, $c \mu^{\prime}+(1-c) \nu^{\prime} \in B R_{i}(\sigma)$. Thus,

$$
\rho^{w}\left(c \mu+(1-c) \nu, B R_{i}(\sigma)\right) \leq \frac{2}{k}
$$

which implies $c \mu+(1-c) \nu \in A B R_{i}^{k}(\sigma)$.

III. We show that $A B R^{k}$ is upper-hemicontinuous. Take sequences $\left(\sigma^{m}\right)_{m=1}^{\infty}$ and $\left(\tau^{m}\right)_{m=1}^{\infty}$ in $\Delta^{k}(\varepsilon)$ such that $\lim _{m \rightarrow \infty} \sigma^{m}=\sigma$ and $\lim _{m \rightarrow \infty} \tau^{m}=\tau$ in Euclidean distance, and such that $\tau^{m} \in A B R^{k}\left(\sigma^{m}\right)$ for every $m \in \mathbf{N}$. We show $\tau_{i} \in A B R_{i}^{k}(\sigma)$ for every $i \in N$, so that $\tau \in A B R^{k}(\sigma)$.

Take $m \in \mathbf{N}$. Since $\tau_{i}^{m} \in A B R_{i}^{k}\left(\sigma^{m}\right)$, we have $\rho^{w}\left(\tau_{i}^{m}, B R_{i}\left(\sigma^{m}\right)\right) \leq \frac{2}{k}$. As $B R_{i}\left(\sigma^{m}\right)$ is a closed set with respect to the weak metric, there is a strategy $\mu_{i}^{m} \in B R_{i}\left(\sigma^{m}\right)$ such that $\rho^{w}\left(\tau_{i}^{m}, \mu_{i}^{m}\right) \leq \frac{2}{k}$. Because $\Delta\left(A_{i}\right)$ is sequentially compact with respect to the weak metric, the sequence $\left(\mu_{i}^{m}\right)_{m=1}^{\infty}$ has a subsequence $\left(\mu_{i}^{m_{\ell}}\right)_{\ell=1}^{\infty}$ with $\rho^{w}\left(\mu_{i}^{m_{\ell}}, \mu_{i}\right) \rightarrow 0$ as $l \rightarrow \infty$ for some $\mu_{i} \in \Delta\left(A_{i}\right)$. Then, by continuity of $u_{i}$ with respect to $\rho^{w}$, we have $\mu_{i} \in B R_{i}(\sigma)$. Moreover, $\rho^{w}\left(\tau_{i}, \mu_{i}\right) \leq \frac{2}{k}$. Hence, $\rho^{w}\left(\tau_{i}, B R_{i}(\sigma)\right) \leq \frac{2}{k}$. This shows that $A B R^{k}$ is upper-hemicontinuous. This concludes III.

By I, II, and III we know that each $A B R^{k}$ satisfies the conditions of Theorem 4.6.6. Hence, for every $k$, there is a $\sigma^{k} \in \Delta^{k}(\varepsilon)$ such that $\sigma^{k} \in A B R^{k}\left(\sigma^{k}\right)$, which 
means that $\sigma_{i}^{k} \in A B R_{i}^{k}\left(\sigma^{k}\right)$ for every $i$. Hence, $\rho^{w}\left(\sigma_{i}^{k}, B R_{i}\left(\sigma^{k}\right)\right) \leq \frac{2}{k}$ for every $i$. Since $\Delta(A)$ is sequentially compact with respect to the weak metric, we can assume w.l.o.g. that the sequence $\sigma^{k}=\left(\sigma_{i}^{k}\right)_{i \in N}$ converges to a strategy profile $\sigma=\left(\sigma_{i}\right)_{i \in N}$ when $k \rightarrow \infty$. Note that $\rho^{w}\left(\sigma_{i}^{k}, B R_{i}\left(\sigma^{k}\right)\right) \rightarrow 0$ as $k \rightarrow \infty$. Hence, $\sigma=\left(\sigma_{i}\right)_{i \in N}$ is a weakly glof perfect equilibrium.

It remains to show that every weakly glof perfect equilibrium is a Nash equilibrium. Suppose that $\sigma=\left(\sigma_{i}\right)_{i \in N}$ is a weakly glof perfect equilibrium. Then, by definition, for every $i$ there is a sequence $\left(\sigma_{i}^{k}\right)_{k=1}^{\infty}$ such that $\rho^{w}\left(\sigma_{i}^{k}, \sigma_{i}\right) \rightarrow 0$ and $\rho^{w}\left(\sigma_{i}^{k}, B R_{i}\left(\sigma^{k}\right)\right) \rightarrow 0$ as $k \rightarrow \infty$. Therefore, for every $i$ there is also a sequence $\left(\mu_{i}^{k}\right)_{k=1}^{\infty}$ such that $\mu_{i}^{k} \in B R_{i}\left(\sigma^{k}\right)$ for every $k \in \mathbf{N}$ and $\rho^{w}\left(\sigma_{i}^{k}, \mu_{i}^{k}\right) \rightarrow 0$ as $k \rightarrow \infty$. By the triangle inequality for $\rho^{w}$, this implies that for every $i, \rho^{w}\left(\mu_{i}^{k}, \sigma_{i}\right) \rightarrow 0$ as $k \rightarrow \infty$. Hence, again by continuity of $u_{i}$, we obtain $\sigma_{i} \in B R_{i}(\sigma)$ for every $i \in N$, as desired.

Now, we prove that the set of weakly glof perfect equilibria is closed with respect to the weak metric. Let $\left(\sigma_{\ell}\right)_{\ell=1}^{\infty}$ be a sequence of weakly glof perfect equilibria in which $\sigma_{\ell}=\left(\sigma_{\ell, 1}, \ldots, \sigma_{\ell, n}\right)$ and suppose that for every player $i$ we have $\rho^{w}\left(\sigma_{\ell, i}, \sigma_{i}\right) \rightarrow 0$ as $\ell \rightarrow \infty$. We show that $\sigma=\left(\sigma_{1}, \ldots, \sigma_{n}\right)$ is weakly glof perfect. Since for every $\ell \in \mathbf{N}$ the strategy profile $\sigma_{\ell}$ is weakly glof perfect, for every $\ell$ and every player $i$, there exist $\left(B_{\ell, i}^{k}\right)_{k=1}^{\infty}$ and $\left(\sigma_{\ell, i}^{k}\right)_{k=1}^{\infty}$ satisfying the conditions of Definition 4.3. Hence, there is a $K_{\ell}^{1}$ such that for every $k \geq K_{\ell}^{1}$, we have $\rho^{w}\left(\sigma_{\ell, i}^{k}, \sigma_{\ell, i}\right)<\frac{1}{\ell}$. Similarly, there is a $K_{\ell}^{2}$ such that for every $k \geq K_{\ell}^{2}$ we have $\rho^{w}\left(\sigma_{\ell, i}^{k}, B R_{i}\left(\sigma_{\ell}^{k}\right)\right)<\frac{1}{\ell}$. Let $K_{\ell}=\max \left\{K_{\ell}^{1}, K_{\ell}^{2}\right\}$. Notice that one can make $K_{\ell}$ large enough such that for every $a_{i} \in A_{i}$ there is a $b_{i} \in B_{i}^{K_{\ell}}$ with $d_{A_{i}}\left(a_{i}, b_{i}\right)<\frac{1}{\ell}$, for every player $i$. Now, the sequence $\left(B_{\ell, i}^{K_{\ell}}\right)_{\ell=1}^{\infty}$ of finite subsets of $A_{i}$ and $\left(\sigma_{\ell, i}^{K_{\ell}}\right)_{\ell=1}^{\infty}$ of completely mixed strategies in $\Delta\left(B_{\ell, i}^{K_{\ell}}\right)$ satisfy the conditions of Definition 4.3 for the strategy profile $\sigma$ when $\ell \rightarrow \infty$. Namely, for every player $i$ we have

$$
\rho^{w}\left(\sigma_{\ell, i}^{K_{\ell}}, \sigma_{i}\right) \leq \rho^{w}\left(\sigma_{\ell, i}^{K_{\ell}}, \sigma_{\ell, i}\right)+\rho^{w}\left(\sigma_{\ell, i}, \sigma_{i}\right)<\frac{1}{\ell}+\rho^{w}\left(\sigma_{\ell, i}, \sigma_{i}\right),
$$

and

$$
\rho^{w}\left(\sigma_{\ell, i}^{K_{\ell}}, B R_{i}\left(\sigma_{\ell}^{K_{\ell}}\right)\right)<\frac{1}{\ell},
$$

both converge to 0 when $\ell \rightarrow \infty$.

\section{Proofs for Section 5}

Lemma 2.9.1 Suppose that $\tau_{i}$ dominates $\sigma_{i}$. Then there is an $a_{-i} \in A_{-i}$ such that $u_{i}\left(a_{-i}, \tau_{i}\right)>u_{i}\left(a_{-i}, \sigma_{i}\right)$. 
Proof. Suppose that $u_{i}\left(a_{-i}, \tau_{i}\right)=u_{i}\left(a_{-i}, \sigma_{i}\right)$ for every $a_{-i} \in A_{-i}$. Then, for every $\sigma_{-i} \in \Delta\left(A_{-i}\right)$ we have $u_{i}\left(\sigma_{-i}, \tau_{i}\right)=u_{i}\left(\sigma_{-i}, \sigma_{i}\right)$. Contradiction.

Lemma 2.9.2 Suppose that strategy $\tau_{i} \in \Delta\left(A_{i}\right)$ dominates $\sigma_{i} \in \Delta\left(A_{i}\right)$. Then

$$
u_{i}\left(\sigma \mid \tau_{i}\right)>u_{i}\left(\sigma \mid \sigma_{i}\right)
$$

for any completely mixed strategy profile $\sigma \in \Delta(A)$. Consequently, any best response to a completely mixed strategy profile is undominated.

Proof. This is a direct consequence of Lemma 2.9.1 and the continuity of the payoff function $u_{i}$ on $\Delta(A)$.

The next Theorem is a direct consequence of the continuity of measure on monotonic sequences.

Lemma 2.9.3 Suppose that $f$ is a real function on $X$ which is strictly positive and $\mu$ is a measure on $X$ such that $\mu(X)>0$. Then, $\int_{X} f d \mu>0$.

Proposition 2.9.4 The set $D_{i}$ is closed with respect to the weak metric in $\Delta\left(A_{i}\right)$ and $P D_{i}$ is closed in $A_{i}$. Moreover, $D_{i}=\Delta\left(P D_{i}\right)$.

Proof. Assume that $D_{i}$ is non-empty, otherwise the statements are obvious. Take a $\rho_{i} \in D_{i}$. For every $\sigma_{-i} \in \Delta\left(A_{-i}\right)$ define

$$
F_{\sigma_{-i}}=\left\{\sigma_{i} \in \Delta\left(A_{i}\right) \mid u_{i}\left(\sigma_{-i}, \sigma_{i}\right)=u_{i}\left(\sigma_{-i}, \rho_{i}\right)\right\}
$$

Since $u_{i}$ is a continuous function on $\Delta\left(A_{i}\right)$ with respect to the weak metric, $F_{\sigma_{-i}}$ is a closed set. Moreover, $D_{i}=\bigcap_{\sigma_{-i} \in \Delta\left(A_{-i}\right)} F_{\sigma_{-i}}$. Therefore, $D_{i}$ is also a closed set with respect to the weak metric. With a similar argument we can conclude that $P D_{i}$ is a closed subset of $A_{i}$.

Now we show that $D_{i}=\Delta\left(P D_{i}\right)$. Since $\Delta\left(P D_{i}\right) \subseteq D_{i}$, we only need to prove that $\rho_{i} \in D_{i}$ implies $\rho_{i} \in \Delta\left(P D_{i}\right)$, or equivalently $\rho_{i}\left(P D_{i}\right)=1$. Suppose by way of contradiction that $\rho_{i}\left(P D_{i}\right)<1$, implying $\rho_{i}\left(A_{i} \backslash P D_{i}\right)>0$. Take a completely mixed strategy profile $\tau \in \Delta(A)$. Let $c=u_{i}\left(\tau \mid \rho_{i}\right)$. By Lemma 2.9.2 we have

$$
u_{i}\left(\tau \mid x_{i}\right)<u_{i}\left(\tau \mid \rho_{i}\right)=c
$$


for every $x_{i} \in A_{i} \backslash P D_{i}$, while $u_{i}\left(\tau \mid x_{i}\right)=u_{i}\left(\tau \mid \rho_{i}\right)=c$ for every $x_{i} \in P D_{i}$. Then, using Lemma 2.9.3 to get the strict inequality, we have

$$
\begin{aligned}
c=u_{i}\left(\tau \mid \rho_{i}\right) & =\int_{A_{i}} u_{i}\left(\tau \mid x_{i}\right) d \rho_{i} \\
& =\int_{P D_{i}} u_{i}\left(\tau \mid x_{i}\right) d \rho_{i}+\int_{A_{i} \backslash P D_{i}} u_{i}\left(\tau \mid x_{i}\right) d \rho_{i} \\
& <c \rho_{i}\left(P D_{i}\right)+c \rho_{i}\left(A_{i} \backslash P D_{i}\right)=c
\end{aligned}
$$

which is a contradiction. 



\section{Chapter 3}

\section{Behavioral perfect equilibrium in Bayesian games}

\subsection{Introduction}

As in games with incomplete information, for example in auction models, there is a need for a selection tool to rule out less intuitive equilibria, we examine possible extensions of perfect equilibrium to such games, specifically to Bayesian games. In a Bayesian game, first each player receives a private information, called the type of this player, which is drawn according to a known probability distribution. Subsequently, depending on his own type, each player chooses an action, and then receives a payoff depending on the types and the chosen actions of all players. We assume that the set of types and the set of actions are both metric spaces, but we generally do not require that they are compact and also do not require that the payoff functions are continuous. Our intention is to keep the model as wide as possible, so that it includes various applications.

We base our investigation on so-called behavioral strategies, which are much in the spirit of Bayesian games. A behavioral strategy for a player prescribes a probability measure on his set of actions, depending on the type of this player, and only satisfies an additional measurability assumption. A central solution concept in these Bayesian games is the concept of Bayesian Nash Equilibrium (BNE), which is a direct extension of Nash equilibrium. A BNE is a profile of behavioral strategies, one for each player, such that each player's strategy, given any type for this player, is in expectation a best response to the strategies of his opponents, where in the expectation all possible types of the opponents have to be taken into account. So, the best response property is required at the interim stage when the player already knows his own type. We remark that a similar but weaker concept arises if one calculates 
ex-ante probabilities, and only requires the best response property for each player before he receives his own type. This approach is investigated for example by Reny [40] and by Milgrom and Weber [33]. From now on, we will work with ex-interim probabilities, unless mentioned otherwise.

In this chapter, our goal is to define the notion of perfect BNE in Bayesian games, and analyze its properties. We base our approach on completely mixed behavioral strategies, i.e. behavioral strategies which prescribe a completely mixed probability measure for each possible type. As a first step, we define perfection for a behavioral strategy profile, so not necessarily for a BNE yet. We define three versions of perfection, which differ in the kind of convergence over the set of types. The main idea roughly is that a behavioral strategy profile $\beta$ is called perfect, if there is a sequence $\left(\beta^{k}\right)_{k=1}^{\infty}$ of completely mixed behavioral strategy profiles such that, for every player $i$, the distance between $\beta_{i}^{k}$ and $\beta_{i}$ and the distance between $\beta_{i}^{k}$ and player $i$ 's best responses against $\beta^{k}$ both converge to 0 . Here, by convergence we mean convergence on the set of actions with respect to the weak metric, and convergence on the set of types in one of the following three senses: uniformly for all types, pointwisely for each type, and almost everywhere pointwisely. Respectively, we call $\beta$ uniform-perfect, pointwise-perfect, or a.e.-pointwise-perfect. In general, such a profile $\beta$ is not necessarily a BNE. If $\beta$ is also a BNE, then we call $\beta$ a uniform-perfect, pointwise-perfect, or a.e.-pointwise-perfect BNE, respectively.

We analyze each of these three notions of perfection in Bayesian games in detail, and we discuss the relations between them. We also pay attention to special cases, mainly when compactness is imposed on the type and action spaces or when the payoff functions satisfy some kind of continuity property. We also devote a small section to finite Bayesian games, i.e. when there are only finitely many types and actions.

In the second part of this chapter, we illustrate that perfection can be a useful tool to select the more intuitive BNEs in Bayesian games. We do so by examining a sealed-bid second-price auction with two bidders. In this auction, the valuation function of each bidder does not only depend on his own type, but also on type of his opponent. More precisely, for each $i=1,2$, bidder $i$ 's valuation is given by $v_{i}=5+t_{i}-\alpha t_{j}$, where $\alpha \in(0,1)$ and $j \neq i$. Jackson et al. [16] and [17] considered the first price version of this auction with $\alpha=4$ and proved that no BNE exists if each player has a positive probability to win in case of a tie.

This second-price auction admits multiple BNEs, but we find that perfection selects a $\mathrm{BNE} \beta$ that is unique in a certain class of strategies and rules out various BNEs which are less intuitive. We highlight that the choice of sequence of com- 
pletely mixed behavioral strategy profiles $\left(\beta^{k}\right)_{k=1}^{\infty}$ is a very subtle task, because the most straightforward candidate for this sequence does not work. We also provide additional results, such as that $\beta$ is not sincere and that neither player has a dominant strategy in this auction.

This chapter is structured as follows. First, we discuss some preliminary notions in section 3.2 and present the model of Bayesian games in section 3.3. Then, in section 3.4 we define the concept of perfect BNE and analyze its properties. In section 3.5 , we apply our results to the above mentioned auction. The chapter ends with an extensive appendix, which contains the proofs of several technical results that we use in the earlier part of the chapter.

\subsection{The model}

A Bayesian game is a tuple $\Gamma=\left(N,\left(T_{i}\right)_{i \in N},\left(A_{i}\right)_{i \in N},\left(\mu_{i}\right)_{i \in N},\left(\Pi_{i}\right)_{i \in N}\right)$ where:

1. $N=\{1,2, . ., n\}$ is the set of players.

2. $T_{i}$ is a nonempty set of player $i$ 's possible types with metric $d_{T_{i}}$. Let $\mathcal{T}_{i}$ denote the induced Borel $\sigma$-field on $T_{i}, T=\times_{i=1}^{n} T_{i}$, and $\mathcal{T}=\otimes_{i=1}^{n} \mathcal{T}_{i}$.

3. $A_{i}$ is a nonempty set of player $i$ 's actions with metric $d_{A_{i}}$. Let $\mathcal{A}_{i}$ denote the induced Borel $\sigma$-field on $A_{i}, A=\times{ }_{i=1}^{n} A_{i}$, and $\mathcal{A}=\otimes_{i=1}^{n} \mathcal{A}_{i}$.

4. $\mu_{i}$ is a probability measure on $\left(T_{i}, \mathcal{T}_{i}\right)$ for player $i$. Let $\mu=\times_{i=1}^{n} \mu_{i}$ be the product measure on $(T, \mathcal{T})$.

5. $\Pi_{i}: T \times A \rightarrow \mathbf{R}$ is player $i$ 's payoff function, bounded and measurable with respect to $\mathcal{T} \otimes \mathcal{A}$. Let $\Pi=\left(\Pi_{i}\right)_{i=1}^{n}$.

The Bayesian game $\Gamma$ is played as follows: First, nature draws a type $t_{i} \in T_{i}$ for each player $i$ according to the probability measure $\mu_{i}$. Each player $i$ learns his own type $t_{i}$, but not the types of the other players. Then, each player $i$ chooses an action $a_{i} \in A_{i}$, simultaneously and independently. Finally, depending on the types $t=\left(t_{1}, \ldots, t_{n}\right)$ and the chosen actions $a=\left(a_{1}, \ldots, a_{n}\right)$, each player $i$ receives the payoff $\Pi_{i}(t, a)$.

Now, we discuss different classes of strategies for the players. We start with the simplest ones.

Definition 3.2.1 A pure strategy for player $i$ is a measurable function $p_{i}: T_{i} \rightarrow A_{i}$. 
Thus, a pure strategy prescribes one specific action depending on the player's type. Now we move on to the strategies which use some randomization for the choice of an action. In the finite version of the model, i.e., when $T_{i}$ and $A_{i}$ are finite, a mixed strategy is a probability measure on the set of pure strategies, whereas a behavioral strategy prescribes, for each possible type, a probability measure on the set of available actions. Aumann [4] observed however that, for our infinite model, the above view of a mixed strategy leads to measure theoretic problems and does not provide an acceptable definition. Instead, a mixed strategy should be modeled by a random variable with values in the set of pure strategies, whose domain is a probability measure space that is used as the randomization device. This is the underlying idea of the definition of a mixed strategy for player $i$ as a measurable function $\alpha_{i}: T_{i} \times[0,1] \rightarrow A_{i}$, where the uniform distribution is imposed on $[0,1]$. The interpretation of a mixed strategy $\alpha_{i}$ is that, after observing his own type $t_{i}$ and drawing a randomization-variable $s_{i}$ from $[0,1]$ according to uniform distribution, player $i$ plays $\alpha_{i}\left(t_{i}, s_{i}\right)$. Note that, for every $s_{i} \in[0,1]$, the section function $\alpha_{i}\left(\cdot, s_{i}\right): T_{i} \rightarrow A_{i}$ is a pure strategy. Behavioral strategies are defined with similar considerations:

Definition 3.2.2 (Milgrom and Weber) A behavioral strategy for player $i$ is a function $\beta_{i}: T_{i} \times \mathcal{A}_{i} \rightarrow[0,1]$ such that

1. the section function $\beta_{i}\left(t_{i}, \cdot\right): \mathcal{A}_{i} \rightarrow[0,1]$ is a probability measure for every $t_{i} \in T_{i}$,

2. the section function $\beta_{i}(\cdot, B): T_{i} \rightarrow[0,1]$ is measurable for every $B \in \mathcal{A}_{i}$.

A behavioral strategy $\beta_{i}$ prescribes, depending on player $i$ 's type $t_{i}$, to choose an action according to the probability measure $\beta_{i}\left(t_{i}, \cdot\right)$. The second condition in the definition is included so that the ex-ante probability that player $i$ 's action falls into a set $B \in \mathcal{A}_{i}$ exists and is equal to $\int_{T_{i}} \beta_{i}\left(t_{i}, B\right) \mu_{i}\left(d t_{i}\right)$. We will usually define a behavioral strategy by specifying the section function $\beta_{i}\left(t_{i}, \cdot\right)$ for every type $t_{i} \in T_{i}$. Behavioral strategies are well suited for our purpose to define perfect equilibrium. Indeed, the probability measures $\beta_{i}\left(t_{i}, \cdot\right)$ are sufficient to describe player $i$ 's behavior. Moreover, as Aumann [4] showed, there is a many-to-one mapping from mixed to behavioral strategies that preserves the players' expected payoffs, so mixed strategies would have no significant added value. For these reasons, we build our definitions on behavioral strategies. From now on, by a strategy we will always mean a behavioral strategy, unless mentioned otherwise. 
Definition 3.2.3 A strategy $\beta_{i}$ for player $i$ is called deterministic if, for every type $t_{i} \in T_{i}$, there is an action $a_{i, t_{i}} \in A_{i}$ such that $\beta_{i}\left(t_{i}, \cdot\right)$ is the Dirac measure on $a_{i, t_{i}}$. A strategy $\beta_{i}$ is called completely mixed if the section function $\beta_{i}\left(t_{i}, \cdot\right)$ : $\mathcal{A}_{i} \rightarrow[0,1]$ is a completely mixed probability measure for every $t_{i} \in T_{i}$. The vector $\beta=\left(\beta_{1}, \beta_{2}, \ldots, \beta_{n}\right)$, where $\beta_{i}$ is a strategy of player $i$, is called a strategy profile.

Each pure strategy $p_{i}$ naturally induces a deterministic strategy $\beta_{i}$, for which $\beta_{i}\left(t_{i}, \cdot\right)$ is the Dirac measure on $p_{i}\left(t_{i}\right)$ for every type $t_{i} \in T_{i}$.

For every strategy profile $\beta$ and every player $i \in N$, we write $\beta_{-i}=\left(\beta_{j}\right)_{j \in N \backslash\{i\}}$ to denote the profile consisting of strategies of the players in $N \backslash\{i\}$. Further, we use $\mathbf{E}_{\tau_{-i}}\left(\Pi_{i} \mid t_{i}, \beta_{i}\right)$ to denote player $i$ 's expected payoff, given his type $t_{i}$ and his strategy $\beta_{i}$, against a strategy profile $\tau_{-i}$. Thus,

$$
\mathbf{E}_{\tau_{-i}}\left(\Pi_{i} \mid t_{i}, \beta_{i}\right)=\int_{T_{-i}} \int_{A_{-i}} \int_{A_{i}} \Pi_{i}(t, a) \beta_{i}\left(t_{i}, d a_{i}\right) \tau_{-i}\left(t_{-i}, d a_{-i}\right) \mu_{-i}\left(d t_{-i}\right) .
$$

In the expected payoff above, i.e. $\mathbf{E}_{\tau_{-i}}\left(\Pi_{i} \mid t_{i}, \beta_{i}\right)$, by integrals with respect to $\tau_{-i}\left(t_{-i}, d a_{-i}\right)$ and $\mu_{-i}\left(d t_{-i}\right)$ we mean the iterated integrals with respect to $\tau_{j}\left(t_{j}, d a_{j}\right)$ and $\mu_{j}\left(d t_{j}\right)$ for all $j \neq i$. Fubini's Theorem and Theorem 4.6.5 in the appendix guarantee the existence of the iterated integrals in the expression above, and also that the order of integration with respect to $\beta_{i}\left(t_{i}, d a_{i}\right)$ and $\tau_{j}\left(t_{j}, d a_{j}\right)$, $j \neq i$, is not relevant. In the special case where player $i$ uses a deterministic strategy with corresponding pure strategy $p_{i}$, player $i$ 's expected payoff is denoted simply by $\mathbf{E}_{\tau_{-i}}\left(\Pi_{i} \mid t_{i}, p_{i}\right)$, and it is equal to

$$
\mathbf{E}_{\tau_{-i}}\left(\Pi_{i} \mid t_{i}, p_{i}\right)=\int_{T_{-i}} \int_{A_{-i}} \Pi_{i}\left(t,\left(p_{i}\left(t_{i}\right), a_{-i}\right)\right) \tau_{-i}\left(t_{-i}, d a_{-i}\right) \mu_{-i}\left(d t_{-i}\right) .
$$

Since in the expected payoffs $\mathbf{E}_{\tau_{-i}}\left(\Pi_{i} \mid t_{i}, \beta_{i}\right)$ and $\mathbf{E}_{\tau_{-i}}\left(\Pi_{i} \mid t_{i}, p_{i}\right)$ it is irrelevant how player $i$ chooses his actions for types other than $t_{i}$, we can naturally define $\mathbf{E}_{\tau_{-i}}\left(\Pi_{i} \mid t_{i}, \sigma_{i}\right)$ and $\mathbf{E}_{\tau_{-i}}\left(\Pi_{i} \mid t_{i}, a_{i}\right)$ for every probability measure $\sigma_{i}$ on $\left(A_{i}, \mathcal{A}_{i}\right)$ and respectively for every action $a_{i} \in A_{i}$.

A probability measure $\sigma_{i}$ on $\left(A_{i}, \mathcal{A}_{i}\right)$ is called a best response of player $i$ for type $t_{i} \in T_{i}$ against a strategy profile $\tau_{-i}$, if for every probability measure $\sigma_{i}^{\prime}$ on $\left(A_{i}, \mathcal{A}_{i}\right)$ we have

$$
\mathbf{E}_{\tau_{-i}}\left(\Pi_{i} \mid t_{i}, \sigma_{i}\right) \geq \mathbf{E}_{\tau_{-i}}\left(\Pi_{i} \mid t_{i}, \sigma_{i}^{\prime}\right)
$$

The set of such best responses is denoted by $B R_{i}\left(t_{i}, \tau_{-i}\right)$. For a strategy profile $\tau$, we will also use the notation $B R_{i}\left(t_{i}, \tau\right)$ instead of $B R_{i}\left(t_{i}, \tau_{-i}\right)$.

A strategy $\beta_{i}$ is called a best response of player $i$ against a strategy profile $\tau_{-i}$, if $\beta_{i}\left(t_{i}, \cdot\right) \in B R_{i}\left(t_{i}, \tau_{-i}\right)$ for every $t_{i} \in T_{i}$. The set of such best responses is denoted 
by $B R_{i}\left(\tau_{-i}\right)$. For a strategy profile $\tau$, we will also use the notation $B R_{i}(\tau)$ instead of $B R_{i}\left(\tau_{-i}\right)$. Note that all these best response sets can be empty, which is illustrated by the following simple example.

Example 3.2.4 Consider the following Bayesian game with only one player: $T_{1}=$ $\left\{t_{1}\right\}, A_{1}=[0,1], \Pi_{1}\left(t_{1}, x\right)=x$ for every $x \in[0,1)$, and $\Pi_{1}\left(t_{1}, 1\right)=0$. In this game, the set of the best responses (that is, optimal strategies) of player 1 is empty.

Now we define a central solution concept of Bayesian games, namely the concept of Bayesian Nash equilibrium.

Definition 3.2.5 A strategy profile $\beta=\left(\beta_{1}, \beta_{2}, \ldots, \beta_{n}\right)$ is called a Bayesian Nash equilibrium (BNE), if $\beta_{i}$ is a best response of player $i$ against $\beta$, for every player $i$.

There are Bayesian games which admit no BNE at all, for instance the game in Example 3.2.4. In many Bayesian games of economic interest, however, there exist multiple BNEs, and some of them are arguably more intuitive than others. As mentioned before, our goal is to develop the definition of perfection for BNEs, which can be a useful tool in such games to distinguish the more intuitive BNEs.

\subsection{Behavioral perfect Bayesian Nash equilibrium}

In this section we propose a number of possible definitions of perfect BNE for Bayesian games. We introduce three main definitions. In each of them, a different notion of convergence is used on the set of strategies. At the end of the section, we draw some conclusions in the context of finite games, i.e. when there are only finitely many types and actions.

\section{Uniform-perfect BNE}

Definition 3.3.1 A strategy profile $\beta=\left(\beta_{1}, \ldots, \beta_{n}\right)$ is called uniform-perfect, if there exists a sequence of profiles of completely mixed strategies $\left(\beta^{k}\right)_{k=1}^{\infty}=$ $\left(\beta_{1}^{k}, \ldots, \beta_{n}^{k}\right)_{k=1}^{\infty}$ with the following properties for every player $i$ :

(1) $\lim _{k \rightarrow \infty} \sup _{t_{i} \in T_{i}} \rho^{w}\left(\beta_{i}^{k}\left(t_{i}, \cdot\right), \beta_{i}\left(t_{i}, \cdot\right)\right)=0$,

(2) $\lim _{k \rightarrow \infty} \sup _{t_{i} \in T_{i}} \rho^{w}\left(\beta_{i}^{k}\left(t_{i}, \cdot\right), B R_{i}\left(t_{i}, \beta^{k}\right)\right)=0$.

A strategy profile $\beta$ is called a uniform-perfect BNE if $\beta$ is a BNE and uniformperfect. 
Note that conditions (1) and (2) in the above definition require a uniform convergence of the corresponding distances over the set of possible types. In the special case when the game has complete information, i.e. each $T_{i}$ is a singleton, our definition coincides with Simon and Stinchcombe's weak perfect equilibrium in [42].

A uniform-perfect strategy profile, and therefore a uniform-perfect BNE, does not always exist, which is for instance the case in Example 3.2.4, because the best response set in this game is always empty. Furthermore, even if a uniform-perfect strategy profile exists, it is not necessarily a BNE, which is illustrated by the following example.

Example 3.3.2 Consider the following Bayesian game with only one player: $T_{1}=$ $\left\{t_{1}\right\}, A_{1}=[0,1], \Pi_{1}\left(t_{1}, x\right)=1$ for every $x \in[0,1)$, and $\Pi_{1}\left(t_{1}, 1\right)=0$. Define strategies $\beta_{1}$ and $\beta_{1}^{k}$, for every $k \in \mathbf{N}$, as follows: $\beta_{1}\left(t_{1}, \cdot\right)=\delta_{1}(\cdot)$ and $\beta_{1}^{k}\left(t_{1}, \cdot\right)=$ $\left(1-\frac{1}{k}\right) \delta_{1-\frac{1}{k}}(\cdot)+\frac{1}{k} \sigma(\cdot)$, for every $k \in \mathbf{N}$, where $\delta_{x}$ is the Dirac measure on action $x$ and $\sigma$ is the uniform distribution on the Borel sets of $[0,1]$. Note that the set of the pure best responses (that is, optimal strategies) of player 1 consists of all actions in $[0,1)$. So, the strategy $\beta_{1}$ and the sequence of completely mixed strategies $\left(\beta_{1}^{k}\right)_{k=1}^{\infty}$ satisfy conditions (1) and (2) of Definition 3.3.1. Therefore, $\beta_{1}$ is a uniform-perfect strategy profile, but it is clearly not a BNE.

A uniform-perfect strategy profile may fail to exist, even if the type and action spaces are compact and the payoff functions are continuous, as is shown in Example 3.3.5. Nevertheless, it follows from Theorem 3.3.4 under fairly weak conditions that every uniform-perfect strategy profile is a BNE.

\section{Pointwise-perfect BNE}

Definition 3.3.3 A strategy profile $\beta=\left(\beta_{1}, \ldots, \beta_{n}\right)$ is called pointwise-perfect, if there exists a sequence of profiles of completely mixed strategies $\left(\beta^{k}\right)_{k=1}^{\infty}=$ $\left(\beta_{1}^{k}, \ldots, \beta_{n}^{k}\right)_{k=1}^{\infty}$ with the following properties for every player $i$ and each type $t_{i} \in T_{i}$ :

(1) $\lim _{k \rightarrow \infty} \rho^{w}\left(\beta_{i}^{k}\left(t_{i}, \cdot\right), \beta_{i}\left(t_{i}, \cdot\right)\right)=0$,

(2) $\lim _{k \rightarrow \infty} \rho^{w}\left(\beta_{i}^{k}\left(t_{i}, \cdot\right), B R_{i}\left(t_{i}, \beta^{k}\right)\right)=0$.

A strategy profile $\beta$ is called a pointwise-perfect $\mathrm{BNE}$ if $\beta$ is a $\mathrm{BNE}$ and pointwiseperfect.

Note that conditions (1) and (2) in the above definition require pointwise convergence of the corresponding distances over the set of possible types. 
A pointwise-perfect strategy profile, and therefore a pointwise-perfect BNE, does not always exist, which is for instance the case in Example 3.2.4, because the best response set in this game is always empty. Furthermore, it follows from Example 3.3.2 that a pointwise-perfect strategy profile is not necessarily a BNE.

We do not know if a pointwise-perfect strategy profile always exists under the condition that the type and action spaces are compact and the payoff functions are continuous. Nevertheless, we have the following result.

Theorem 3.3.4 In a Bayesian game where the action spaces are separable and $\Pi_{i}(t, \cdot)$ is continuous on A for every player $i$ and every $t \in T$, every pointwiseperfect strategy profile is a BNE.

Proof. Suppose that $\beta=\left(\beta_{1}, \ldots, \beta_{n}\right)$ is a pointwise-perfect strategy profile in such a Bayesian game. For $\beta$, take a sequence of strategy profiles $\left(\beta^{k}\right)_{k=1}^{\infty}$ as in Definition 3.3.3. Fix a player $i$ and a type $t_{i} \in T_{i}$. Due to condition (2) in Definition 3.3.3, there exists a $K_{i}\left(t_{i}\right) \in \mathbf{N}$ such that $B R_{i}\left(t_{i}, \beta^{k}\right)$ is nonempty for every $k \geq K_{i}\left(t_{i}\right)$. Therefore, by condition (2) once more, there exists a sequence $\left(\sigma_{i, t_{i}}^{k}\right)_{k=1}^{\infty}$ of probability measures on $\left(A_{i}, \mathcal{A}_{i}\right)$ such that $\sigma_{i, t_{i}}^{k} \in B R_{i}\left(t_{i}, \beta^{k}\right)$ for every $k \geq K_{i}\left(t_{i}\right)$ and $\rho^{w}\left(\beta_{i}^{k}\left(t_{i}, \cdot\right), \sigma_{i, t_{i}}^{k}\right) \rightarrow 0$ as $k \rightarrow \infty$. By condition (1) and by the triangle inequality for $\rho^{w}$, this implies that $\rho^{w}\left(\beta_{i}\left(t_{i}, \cdot\right), \sigma_{i, t_{i}}^{k}\right) \rightarrow 0$ as $k \rightarrow \infty$.

For every $k \geq K_{i}\left(t_{i}\right)$ and every probability measure $\sigma^{\prime}$ on $\left(A_{i}, \mathcal{A}_{i}\right)$, we have due to $\sigma_{i, t_{i}}^{k} \in B R_{i}\left(t_{i}, \beta^{k}\right)$ that $\mathbf{E}_{\beta_{-i}^{k}}\left(\Pi_{i} \mid t_{i}, \sigma_{i}^{k}\right) \geq \mathbf{E}_{\beta_{-i}^{k}}\left(\Pi_{i} \mid t_{i}, \sigma^{\prime}\right)$, which means that

$$
\begin{gathered}
\int_{T_{-i}} \int_{A_{-i}} \int_{A_{i}} \Pi_{i}\left(\left(t_{i}, t_{-i}\right), a\right) \sigma_{i, t_{i}}^{k}\left(d a_{i}\right) \beta_{-i}^{k}\left(t_{-i}, d a_{-i}\right) \mu_{-i}\left(d t_{-i}\right) \\
\geq \int_{T_{-i}} \int_{A_{-i}} \int_{A_{i}} \Pi_{i}\left(\left(t_{i}, t_{-i}\right), a\right) \sigma^{\prime}\left(d a_{i}\right) \beta_{-i}^{k}\left(t_{-i}, d a_{-i}\right) \mu_{-i}\left(d t_{-i}\right) .
\end{gathered}
$$

According to Theorem 3.2 in [6], in a separable product space the weak convergence of the product measure is equivalent to the weak convergence of the marginal measures. Hence, because $A$ is separable, the weak convergence of $\sigma_{i, t_{i}}^{k}$ to $\beta_{i}\left(t_{i}, \cdot\right)$ and of $\beta_{j}^{k}\left(t_{j}, \cdot\right)$ to $\beta_{j}\left(t_{j}, \cdot\right)$ for every player $j \neq i$ and every type $t_{j} \in T_{j}$ implies that, for any $t_{-i} \in T_{-i}$, the product measure $\sigma_{i, t_{i}}^{k} \times\left(\times_{j \neq i} \beta_{j}^{k}\left(t_{j}, \cdot\right)\right)$ on $(A, \mathcal{A})$ weakly converges to the product measure $\beta_{i}\left(t_{i}, \cdot\right) \times\left(\times_{j \neq i} \beta_{j}\left(t_{j}, \cdot\right)\right)$. Since the payoff function $\Pi_{i}\left(\left(t_{i}, t_{-i}\right), \cdot\right)$ is continuous on $A$ for every $t_{-i} \in T_{-i}$, by Fubini's theorem we have for every $t_{-i} \in T_{-i}$

$$
\lim _{k \rightarrow \infty} \int_{A_{-i}} \int_{A_{i}} \Pi_{i}\left(\left(t_{i}, t_{-i}\right), a\right) \sigma_{i, t_{i}}^{k}\left(d a_{i}\right) \beta_{-i}^{k}\left(t_{-i}, d a_{-i}\right)
$$




$$
=\int_{A_{-i}} \int_{A_{i}} \Pi_{i}\left(\left(t_{i}, t_{-i}\right), a\right) \beta_{i}\left(t_{i}, d a_{i}\right) \beta_{-i}\left(t_{-i}, d a_{-i}\right),
$$

and similarly

$$
\begin{aligned}
\lim _{k \rightarrow \infty} \int_{A_{-i}} \int_{A_{i}} \Pi_{i}\left(\left(t_{i}, t_{-i}\right), a\right) \sigma^{\prime}\left(d a_{i}\right) \beta_{-i}^{k}\left(t_{-i}, d a_{-i}\right) \\
\quad=\int_{A_{-i}} \int_{A_{i}} \Pi_{i}\left(\left(t_{i}, t_{-i}\right), a\right) \sigma^{\prime}\left(d a_{i}\right) \beta_{-i}\left(t_{-i}, d a_{-i}\right) .
\end{aligned}
$$

Thus, if we take the limit in inequality (3.1) when $k \rightarrow \infty$, by the dominated convergence theorem we obtain

$$
\begin{aligned}
\int_{T_{-i}} \int_{A_{-i}} \int_{A_{i}} \Pi_{i}(t, a) \beta_{i}\left(t_{i}, d a_{i}\right) \beta_{-i}\left(t_{-i}, d a_{-i}\right) \mu_{-i}\left(d t_{-i}\right) \\
\geq \int_{T_{-i}} \int_{A_{-i}} \int_{A_{i}} \Pi_{i}(t, a) \sigma^{\prime}\left(d a_{i}\right) \beta_{-i}\left(t_{-i}, d a_{-i}\right) \mu_{-i}\left(d t_{-i}\right),
\end{aligned}
$$

which means $\mathbf{E}_{\beta_{-i}}\left(\Pi_{i} \mid t_{i}, \beta_{i}\left(t_{i}, \cdot\right)\right) \geq \mathbf{E}_{\beta_{-i}}\left(\Pi_{i} \mid t_{i}, \sigma^{\prime}\right)$. Hence, $\beta_{i}\left(t_{i}, \cdot\right) \in B R_{i}\left(t_{i}, \beta\right)$. Since player $i$ and type $t_{i}$ were chosen arbitrary, the strategy profile $\beta$ is a BNE as claimed.

It is clear that every uniform-perfect BNE is also pointwise-perfect. Now, we provide a game which demonstrates that the opposite is not always true. This game has a pointwise-perfect BNE, but it has no uniform-perfect BNE, in fact not even a uniform-perfect strategy profile, although the type and action spaces are compact and the payoff functions are continuous.

Example 3.3.5 Consider the following Bayesian game with two players: Player 1 has only one possible type, $T_{1}=\left\{t_{1}\right\}$, whereas player 2 's type space is $T_{2}=$ $\left\{t_{2}^{1}, t_{2}^{2}, \ldots, t_{2}^{\infty}\right\}$ in which $t_{2}^{\infty}$ is the limit point of the sequence $\left(t_{2}^{m}\right)_{m=1}^{\infty}$. The probability measure $\mu_{2}$ on $\left(T_{2}, \mathcal{T}_{2}\right)$ is arbitrary. The action spaces are $A_{1}=\{U, D\}$ and $A_{2}=\{L, R\}$. The payoff matrix when player 1 is the row-player and is given type $t_{1}$, and player 2 is the column-player and is given type $t_{2}^{m}$, for every $m \in \mathbf{N}$, is the following:

\begin{tabular}{r|rr}
$\left(t_{1}, t_{2}^{m}\right)$ & $L$ & $R$ \\
\hline $\mathrm{U}$ & $0,-\frac{1}{m}$ & 0,0 \\
$\mathrm{D}$ & $1, \frac{1}{m^{2}}$ & 1,0
\end{tabular}


and the payoff matrix when player 1 is given type $t_{1}$ and player 2 is given type $t_{2}^{\infty}$ is:

\begin{tabular}{r|rr}
$\left(t_{1}, t_{2}^{\infty}\right)$ & $L$ & $R$ \\
\hline $\mathrm{U}$ & 0,0 & 0,0 \\
$\mathrm{D}$ & 1,0 & 1,0
\end{tabular}

Observe that the type and action spaces in this game are compact, and the payoff functions are continuous.

Define $p_{1}\left(t_{1}\right)=D$ and $p_{2}\left(t_{2}^{m}\right)=L$, for every $m \in \mathbf{N} \cup\{\infty\}$. Let $\beta_{1}$ and $\beta_{2}$ be the deterministic strategies corresponding to $p_{1}$ and $p_{2}$ respectively. We claim that $\left(\beta_{1}, \beta_{2}\right)$ is a pointwise-perfect $\mathrm{BNE}$, but the game has no uniform-perfect strategy profile, so no uniform-perfect BNE either.

The proof that $\left(\beta_{1}, \beta_{2}\right)$ is a pointwise-perfect BNE: Clearly, $\left(\beta_{1}, \beta_{2}\right)$ is a $\mathrm{BNE}$, so it remains to prove that $\left(\beta_{1}, \beta_{2}\right)$ is pointwise-perfect. For every $k \in \mathbf{N}$, define two completely mixed strategies $\beta_{1}^{k}$ and $\beta_{2}^{k}$ by letting $\beta_{1}^{k}\left(t_{1}, \cdot\right)=\left(1-\frac{1}{k}\right) \delta_{D}(\cdot)+$ $\frac{1}{k} \delta_{U}(\cdot)$ and $\beta_{2}^{k}\left(t_{2}^{m}, \cdot\right)=\left(1-\frac{1}{k}\right) \delta_{L}(\cdot)+\frac{1}{k} \delta_{R}(\cdot)$ for every $m \in \mathbf{N} \cup\{\infty\}$, where $\delta_{x}$ denotes the Dirac measure on $x$. The sequence $\left(\beta^{k}\right)_{k=1}^{\infty}=\left(\beta_{1}^{k}, \beta_{2}^{k}\right)_{k=1}^{\infty}$ clearly satisfies condition (1) of Definition 3.3.3, and it also satisfies condition (2) for player 1 and condition (2) for type $t_{2}^{\infty}$ of player 2. It remains to verify condition (2) for an arbitrary type $t_{2}^{m}$ for player 2 where $m \in \mathbf{N}$. Take such a type $t_{2}^{m}$. We have

$$
\mathbf{E}_{\beta_{1}^{k}}\left(\Pi_{2} \mid t_{2}^{m}, L\right)=\left(1-\frac{1}{k}\right) \cdot \frac{1}{m^{2}}+\frac{1}{k} \cdot\left(-\frac{1}{m}\right),
$$

which is strictly positive for large $k$. Hence, $B R_{2}\left(t_{2}^{m}, \beta^{k}\right)=\left\{\delta_{L}\right\}$ for large $k$, which implies that condition (2) of Definition 3.3.3 holds for type $t_{2}^{m}$. Thus, $\left(\beta_{1}, \beta_{2}\right)$ is pointwise-perfect indeed.

The proof that this game has no uniform-perfect strategy profile: Suppose by way of contradiction that $\tau=\left(\tau_{1}, \tau_{2}\right)$ is a uniform-perfect strategy profile. Then, there is a sequence of completely mixed strategy profiles $\left(\tau^{k}\right)_{k=1}^{\infty}=\left(\tau_{1}^{k}, \tau_{2}^{k}\right)_{k=1}^{\infty}$ that satisfy conditions (1) and (2) of Definition 3.3.1 for $\tau$. First notice that, by the triangle inequality for $\rho^{w}$, we have

$$
\begin{aligned}
& \sup _{t_{2} \in T_{2}} \rho^{w}\left(\tau_{2}\left(t_{2}, \cdot\right), B R_{2}\left(t_{2}, \tau^{k}\right)\right) \\
\leq & \sup _{t_{2} \in T_{2}}\left[\rho^{w}\left(\tau_{2}^{k}\left(t_{2}, \cdot\right), \tau_{2}\left(t_{2}, \cdot\right)\right)+\rho^{w}\left(\tau_{2}^{k}\left(t_{2}, \cdot\right), B R_{2}\left(t_{2}, \tau^{k}\right)\right)\right] \\
\leq & \sup _{t_{2} \in T_{2}} \rho^{w}\left(\tau_{2}^{k}\left(t_{2}, \cdot\right), \tau_{2}\left(t_{2}, \cdot\right)\right)+\sup _{t_{2} \in T_{2}} \rho^{w}\left(\tau_{2}^{k}\left(t_{2}, \cdot\right), B R_{2}\left(t_{2}, \tau^{k}\right)\right),
\end{aligned}
$$

and therefore conditions (1) and (2) imply

$$
\lim _{k \rightarrow \infty} \sup _{t_{2} \in T_{2}} \rho^{w}\left(\tau_{2}\left(t_{2}, \cdot\right), B R_{2}\left(t_{2}, \tau^{k}\right)\right)=0
$$


For every $k \in \mathbf{N}$, the probability measure $\tau_{1}^{k}\left(t_{1}, \cdot\right)$ can be written in the form $\tau_{1}^{k}\left(t_{1}, \cdot\right)=\left(1-\varepsilon_{k}\right) \delta_{U}(\cdot)+\varepsilon_{k} \delta_{D}(\cdot)$ with some $\varepsilon_{k} \in(0,1)$. Due to condition (2) for player 1 , we must have that $\varepsilon_{k} \rightarrow 1$ as $k \rightarrow \infty$.

For every $k \in \mathbf{N}$ and every type $t_{2}^{m}$ for player 2 , where $m \in \mathbf{N}$, we have

$$
\mathbf{E}_{\tau_{1}^{k}}\left(\Pi_{2} \mid t_{2}^{m}, L\right)=\varepsilon_{k} \cdot \frac{1}{m^{2}}+\left(1-\varepsilon_{k}\right) \cdot\left(-\frac{1}{m}\right),
$$

whereas $\mathbf{E}_{\tau_{1}^{k}}\left(\Pi_{2} \mid t_{2}^{m}, R\right)=0$. It has two consequences. First, for every $t_{2}^{m}$, where $m \in \mathbf{N}$, we have $B R_{2}\left(t_{2}^{m}, \tau^{k}\right)=\left\{\delta_{L}\right\}$ for large $k$, which in view of (3.2) yields $\tau_{2}\left(t_{2}^{m}, \cdot\right)=\delta_{L}(\cdot)$ for every $t_{2}^{m}$, where $m \in \mathbf{N}$. Second, for every $k \in \mathbf{N}$, we have $B R_{2}\left(t_{2}^{m}, \tau^{k}\right)=\left\{\delta_{R}\right\}$ for large $m$. In conclusion, for every $k \in \mathbf{N}$, if $m$ is large, then $\tau_{2}\left(t_{2}^{m}, \cdot\right)=\delta_{L}(\cdot)$ and $B R_{2}\left(t_{2}^{m}, \tau^{k}\right)=\left\{\delta_{R}\right\}$. This is in contradiction with (3.2), so $\tau$ is not a uniform-perfect strategy profile.

\section{A.e.-pointwise-perfect BNE}

Definition 3.3.6 A strategy profile $\beta=\left(\beta_{1}, \ldots, \beta_{n}\right)$ is called a.e.-pointwise-perfect (where a.e. stands for almost everywhere), if for every player $i$ there exists a set $S_{i} \in \mathcal{T}_{i}$ with $\mu_{i}\left(S_{i}\right)=0$ and a sequence of profiles of completely mixed strategies $\left(\beta^{k}\right)_{k=1}^{\infty}=\left(\beta_{1}^{k}, \ldots, \beta_{n}^{k}\right)_{k=1}^{\infty}$ with the following properties for every player $i$ and every type $t_{i} \in T_{i} \backslash S_{i}$ :

(1) $\lim _{k \rightarrow \infty} \rho^{w}\left(\beta_{i}^{k}\left(t_{i}, \cdot\right), \beta_{i}\left(t_{i}, \cdot\right)\right)=0$,
(2) $\lim _{k \rightarrow \infty} \rho^{w}\left(\beta_{i}^{k}\left(t_{i}, \cdot\right), B R_{i}\left(t_{i}, \beta^{k}\right)\right)=0$.

A strategy profile $\beta$ is called an a.e.-pointwise-perfect $\mathrm{BNE}$ if $\beta$ is a $\mathrm{BNE}$ and a.e.pointwise-perfect.

An a.e.-pointwise-perfect strategy profile, and therefore an a.e.-pointwise-perfect BNE, does not always exist, which is for instance the case in Example 3.2.4, because the best response set in this game is always empty. Furthermore, it follows from Example 3.3.2 that an a.e.-pointwise-perfect strategy profile is not necessarily a BNE.

It is clear that every pointwise-perfect BNE is also a.e.-pointwise-perfect. The following example proves that the converse is not always true.

Example 3.3.7 Consider the following Bayesian game with two players: The type spaces are $T_{1}=\left\{t_{1}\right\}$ and $T_{2}=\left\{t_{2}^{1}, t_{2}^{2}\right\}$, and $\mu_{2}$ is given by $\mu_{2}\left(t_{2}^{1}\right)=1$ and $\mu_{2}\left(t_{2}^{2}\right)=0$. The action spaces are $A_{1}=\{U, D\}$ and $A_{2}=\{L, R\}$. The payoff 
matrix is the following if player 1 is given type $t_{1}$ whereas player 2 is given type $t_{2}^{m}$ for $m=1,2$ :

\begin{tabular}{r|rr}
$\left(t_{1}, t_{2}^{m}\right)$ & $L$ & $R$ \\
\hline $\mathrm{U}$ & 0,1 & 0,0 \\
$\mathrm{D}$ & 1,0 & 1,0
\end{tabular}

Define $p_{1}\left(t_{1}\right)=D, p_{2}\left(t_{2}^{1}\right)=L$ and $p_{2}\left(t_{2}^{2}\right)=R$. Let $\beta_{1}$ and $\beta_{2}$ be the deterministic strategies corresponding to $p_{1}$ and $p_{2}$ respectively. It is clear that $\left(\beta_{1}, \beta_{2}\right)$ is an a.e.pointwise-perfect BNE, by choosing $S_{1}=\emptyset$ and $S_{2}=\left\{t_{2}^{2}\right\}$ in Definition 3.3.6. Yet, $\left(\beta_{1}, \beta_{2}\right)$ is not pointwise-perfect, because player 2 chooses $R$ with probability 1 if he receives type $t_{2}^{2}$.

We remark that a similar example can be made where $T_{2}=[0,1], \mu_{2}$ is the uniform distribution on $T_{2}$, and where the strategy profile is not pointwise-perfect only due to a single type, say type $t_{2}=1$.

The following theorem presents conditions under which there is a strong connection between a.e.-pointwise and pointwise-perfection.

Proposition 3.3.8 Let $\beta$ be an a.e.-pointwise-perfect BNE in a Bayesian game with compact action spaces. Let $S_{i}$, for every player $i$, and $\left(\beta^{k}\right)_{k=1}^{\infty}$ be as in Definition 3.3.6 for $\beta$. Suppose that $S_{i}$ is countable for every player $i$ and $B R_{i}\left(t_{i}, \beta^{k}\right)$ is nonempty for every player $i$, every type $t_{i} \in S_{i}$ and every $k \in N$. Then, there is a pointwise-perfect BNE $\hat{\beta}$ such that $\hat{\beta}_{i}\left(t_{i}, \cdot\right)=\beta_{i}\left(t_{i}, \cdot\right)$ holds for every player $i$ and every type $t_{i} \in T_{i} \backslash S_{i}$.

Proof. For such an a.e.-pointwise-perfect BNE $\beta$, we can construct a desired pointwise-perfect BNE $\hat{\beta}$ as follows. For every player $i \in N$, every type $t_{i} \in S_{i}$, and every $k \in \mathbf{N}$, by assumption of the proposition, we can choose a probability measure $\sigma_{i, t_{i}}^{k} \in B R_{i}\left(t_{i}, \beta^{k}\right)$. Now, define a profile of completely mixed strategies $\hat{\beta}^{k}$, for every $k \in \mathbf{N}$, by letting

$$
\hat{\beta}_{i}^{k}\left(t_{i}, \cdot\right)= \begin{cases}\beta_{i}^{k}\left(t_{i}, \cdot\right) & \text { if } t_{i} \in T_{i} \backslash S_{i} \\ \left(1-\frac{1}{k}\right) \sigma_{i, t_{i}}^{k}(\cdot)+\frac{1}{k} \beta_{i}^{k}\left(t_{i}, \cdot\right) & \text { if } t_{i} \in S_{i}\end{cases}
$$

for every player $i \in N$ and type $t_{i} \in T_{i}$. Note that since for every $B \in \mathcal{A}_{i}$ the maps $\hat{\beta}_{i}^{k}(\cdot, B)$ and $\beta_{i}^{k}(\cdot, B)$ only differ on a countable subset of $T_{i}$, namely the set $S_{i}, \hat{\beta}_{i}^{k}(\cdot, B): T_{i} \rightarrow[0,1]$ is measurable. Therefore, $\hat{\beta}_{i}^{k}$ is a behavioral strategy.

In the remaining part of the proof, we use several fairly known results from analysis, which can all be found in [1]. By assumption, $A_{i}$ is compact in the topology 
induced by the metric $d_{A_{i}}$, so the set $\Delta\left(A_{i}\right)$ of probability measures on $\left(A_{i}, \mathcal{A}_{i}\right)$ is also compact with respect to the topology induced by the weak metric. Consequently, the product space $\times_{i \in N} \times_{t_{i} \in S_{i}} \Delta\left(A_{i}\right)$ is compact in the product topology by Tychonoff's theorem, and because $S_{i}$ is countable, this topology is even metrizable. Therefore, this topological space is sequentially compact, which assures the existence of a subsequence $\left(k_{r}\right)_{r=1}^{\infty}$ so that $\sigma_{i, t_{i}}^{k_{r}}$ converges to some $\sigma_{i, t_{i}} \in \Delta\left(A_{i}\right)$, for every player $i \in N$ and every $t_{i} \in T_{i}$, with respect to the weak metric. So, define a strategy for every player $i \in N$ by

$$
\hat{\beta}_{i}\left(t_{i}, \cdot\right)= \begin{cases}\beta_{i}\left(t_{i}, \cdot\right) & \text { if } t_{i} \in T_{i} \backslash S_{i} \\ \sigma_{i, t_{i}}(\cdot) & \text { if } t_{i} \in S_{i}\end{cases}
$$

for every type $t_{i} \in T_{i}$. With the same argument as for $\hat{\beta}_{i}^{k}$, it is clear that $\hat{\beta}_{i}$ is a behavioral strategy too. Notice that the profile $\hat{\beta}=\left(\hat{\beta}_{i}\right)_{i \in N}$ is a pointwise-perfect equilibrium. Indeed, for the sequence $\hat{\beta}^{k_{r}}$, condition (1) of Definition 3.3.3 is obviously satisfied, and so is condition (2) because $B R_{i}\left(t_{i}, \hat{\beta}^{k_{r}}\right)=B R_{i}\left(t_{i}, \beta^{k_{r}}\right)$ holds due to $\mu_{j}\left(S_{j}\right)=0$ for all players $j$.

The next example shows that the condition that $S_{i}$ is countable is crucial in Proposition 3.3.8 if there are at least three players. We do not know if this condition is also crucial for games with only two players.

Example 3.3.9 Consider the following Bayesian game with three players. The type spaces are $T_{1}=\{0,1\}^{\mathbf{N}}, T_{2}=\left\{t_{2}\right\}$ and $T_{3}=\left\{t_{3}\right\}$. The metric $d_{T_{1}}$ on $T_{1}$ is defined as follows: for $t_{1}, t_{1}^{\prime} \in T_{1}$, if $t_{1}=t_{1}^{\prime}$ then let $d_{T_{1}}\left(t_{1}, t_{1}^{\prime}\right)=0$, otherwise if $m$ is the first coordinate in which $t_{1}$ and $t_{1}^{\prime}$ differ, then let $d_{T_{1}}\left(t_{1}, t_{1}^{\prime}\right)=2^{-m}$. Notice that $d_{T_{1}}$ induces the product topology on $T_{1}$. Further, let $\mu_{1}=\delta_{(1,1, \ldots)}$, i.e. the Dirac measure on the type $(1,1, \ldots)$. The action spaces are $A_{1}=\{U, D\}, A_{2}=$ $\left\{a_{1}, a_{2}, \ldots, a_{\infty}\right\}$, and $A_{3}=\left\{b_{1}, b_{2}, \ldots, b_{\infty}\right\}$, in which $a_{m}=b_{m}=1-\frac{1}{m}$ for every $m \in \mathbf{N}$ and $a_{\infty}=b_{\infty}=1$. For every type $t_{1} \in\{0,1\}^{\mathbf{N}}$, let $f_{m}\left(t_{1}\right)$ be the $m$-th coordinate of the sequence $t_{1}$. When player 1 is given type $t_{1} \in\{0,1\}^{\mathbf{N}} \backslash\{1,1, \ldots\}$, the payoff of player 1 is independent of the action chosen by player 3 , and it is given by

\begin{tabular}{r|cc}
$t_{1} \in\{0,1\}^{\mathbf{N}} \backslash\{(1,1, \ldots)\}$ & $a_{m}$ & $a_{\infty}$ \\
\hline $\mathrm{U}$ & $f_{m}\left(t_{1}\right)$ & 0 \\
$\mathrm{D}$ & $1-f_{m}\left(t_{1}\right)$ & 0
\end{tabular}


whereas if player 1 is given type $t_{1}=(1,1, \ldots)$ :

\begin{tabular}{r|cc}
$t_{1}=(1,1, \ldots)$ & $a_{m}$ & $a_{\infty}$ \\
\hline $\mathrm{U}$ & 0 & 0 \\
$\mathrm{D}$ & 0 & 0
\end{tabular}

The payoffs of players 2 and 3 are independent of the action chosen by player 1, and are given by:

\begin{tabular}{c|cccccc} 
& $b_{1}$ & $b_{2}$ & $b_{3}$ & $b_{4}$ & $\ldots$ & $b_{\infty}$ \\
\hline$a_{1}$ & 0,0 & $0, \frac{1}{2}$ & $0, \frac{1}{3}$ & $0, \frac{1}{4}$ & $\ldots$ & 0,0 \\
$a_{2}$ & $\frac{1}{2}, 0$ & 0,0 & $0, \frac{1}{3}$ & $0, \frac{1}{4}$ & $\ldots$ & 0,0 \\
$a_{3}$ & $\frac{1}{3}, 0$ & $\frac{1}{3}, 0$ & 0,0 & $0, \frac{1}{4}$ & $\ldots$ & 0,0 \\
$a_{4}$ & $\frac{1}{4}, 0$ & $\frac{1}{4}, 0$ & $\frac{1}{4}, 0$ & 0,0 & $\ldots$ & 0,0 \\
$\vdots$ & $\vdots$ & $\vdots$ & $\vdots$ & $\vdots$ & $\vdots$ & $\vdots$ \\
$a_{\infty}$ & 0,0 & 0,0 & 0,0 & 0,0 & $\ldots$ & 0,0
\end{tabular}

In general, if player 2 chooses action $a_{m}$ and player 3 chooses action $b_{\ell}$, with $m, \ell \in$ $\mathbf{N}$, then player 2's payoff is $\frac{1}{m}$ if $m>\ell$, and 0 otherwise, and player 3's payoff is $\frac{1}{\ell}$ if $\ell>m$, and 0 otherwise.

We remark that the type and action spaces in this game are all compact. Since the payoff functions of players 2 and 3 are continuous, and player 1 has only finitely many actions, the best reply set $B R_{i}\left(t_{i}, \beta\right)$ is nonempty for every player $i$, every $t_{i} \in$ $T_{i}$ and every strategy profile $\beta$. We claim: (1) there exists an a.e.-pointwise-perfect equilibrium in this game, but (2) this game admits no pointwise-perfect strategy profile.

An a.e.-pointwise-perfect BNE: First we construct an a.e.-pointwise-perfect BNE. Define $p_{1}\left(t_{1}\right)=U$ for every $t_{1} \in T_{1}, p_{2}\left(t_{2}\right)=a_{\infty}$ and $p_{3}\left(t_{3}\right)=b_{\infty}$. Let $\widetilde{\beta}_{i}$ be the deterministic strategy for each player $i$ corresponding to $p_{i}$. Now we prove that $\widetilde{\beta}=\left(\widetilde{\beta}_{1}, \widetilde{\beta}_{2}, \widetilde{\beta}_{3}\right)$ is an a.e.-pointwise-perfect BNE. Let $S_{1}=T_{1} \backslash\{(1,1, \ldots)\}$ and $S_{2}=S_{3}=\emptyset$. Further, take arbitrary completely mixed probability measures $\sigma_{2}$ and $\sigma_{3}$ on $\left(A_{2}, \mathcal{A}_{2}\right)$ and $\left(A_{3}, \mathcal{A}_{3}\right)$, respectively. For every $k \in \mathbf{N}$, define the completely mixed strategies

$$
\beta_{1}^{k}\left(t_{1}, \cdot\right)=\left(1-\frac{1}{k}\right) \cdot \delta_{U}(\cdot)+\frac{1}{k} \cdot \delta_{D}(\cdot) \text { for every } t_{1} \in T_{1}
$$




$$
\begin{aligned}
& \beta_{2}^{k}\left(t_{2}, \cdot\right)=\left(1-\frac{1}{k^{2}}\right) \cdot \delta_{a_{k}}(\cdot)+\frac{1}{k^{2}} \cdot \sigma_{2}(\cdot) \\
& \beta_{3}^{k}\left(t_{3}, \cdot\right)=\left(1-\frac{1}{k^{2}}\right) \cdot \delta_{b_{k}}(\cdot)+\frac{1}{k^{2}} \cdot \sigma_{3}(\cdot) .
\end{aligned}
$$

If player 2 plays actions $a_{k+1}$ against $\beta_{3}^{k}\left(t_{3}, \cdot\right)$, then he receives at least $\left(1-\frac{1}{k^{2}}\right) \frac{1}{k+1}$, whereas he receives at most $\frac{1}{k+2}$ by playing an action $a_{\ell}$ with $\ell>k+1$ and receives at most $\frac{1}{2 k^{2}}$ by playing an action $a_{\ell}$ with $\ell<k+1$. Thus, for every $k \geq 3$, action $a_{k+1}$ is player 2's (unique) best response to $\beta_{3}^{k}\left(t_{3}, \cdot\right)$, and for similar reasons, action $b_{k+1}$ is player 3's (unique) best response to $\beta_{2}^{k}\left(t_{2}, \cdot\right)$. Based on this observation, one can check easily that $S_{1}, S_{2}, S_{3}$ and the sequence $\beta^{k}=\left(\beta_{1}^{k}, \beta_{2}^{k}, \beta_{3}^{k}\right)$ satisfy the conditions of Definition 3.3.6 for $\widetilde{\beta}$. Thus, $\widetilde{\beta}$ is an a.e.-pointwise-perfect BNE indeed.

No pointwise-perfect strategy profile: Now we claim that there is no pointwiseperfect strategy profile in this game. (Note that $S_{1}$ above is uncountable, so Proposition 3.3.8 does not apply.) Suppose by way of contradiction that $\beta=\left(\beta_{1}, \beta_{2}, \beta_{3}\right)$ is a pointwise-perfect strategy profile, with some sequence $\left(\beta^{k}\right)_{k=1}^{\infty}$ as required in Definition 3.3.3.

First we prove that $\beta_{2}\left(t_{2}, \cdot\right)$ and $\beta_{3}\left(t_{3}, \cdot\right)$ put probability 1 on action $a_{\infty}$ and $b_{\infty}$, respectively, i.e. $\beta_{2}\left(t_{2},\left\{a_{\infty}\right\}\right)=\beta_{3}\left(t_{3},\left\{b_{\infty}\right\}\right)=1$. Suppose by way of contradiction that $\beta_{2}\left(t_{2},\left\{a_{\infty}\right\}\right)<1$; the proof is similar if $\beta_{3}\left(t_{3},\left\{b_{\infty}\right\}\right)<1$. Let $m^{*}=\min \left\{m \in \mathbf{N} \mid \beta_{2}\left(t_{2},\left\{a_{m}\right\}\right)>0\right\}$. Since player 3 can get a positive payoff against $\beta_{2}\left(t_{2}, \cdot\right)$, and $\beta_{3}\left(t_{3}, \cdot\right)$ is a best response to $\beta_{2}\left(t_{2}, \cdot\right)$, we must have $\beta_{3}\left(t_{3},\left\{b_{\infty}\right\}\right)=0$. So, also player 2 can get a positive payoff against $\beta_{3}\left(t_{3}, \cdot\right)$. Let $\ell^{*}=\min \left\{\ell \in \mathbf{N} \mid \beta_{3}\left(t_{3},\left\{b_{\ell}\right\}\right)>0\right\}$. Now, if $m^{*} \leq \ell^{*}$, then action $a_{m^{*}}$ gives payoff zero to player 2 , which is a contradiction as $\beta_{2}\left(t_{2},\left\{a_{m^{*}}\right\}\right)>0$. Similarly, if $\ell^{*} \leq m^{*}$, then action $b_{\ell^{*}}$ gives payoff zero to player 3 , which is a contradiction as $\beta_{3}\left(t_{3},\left\{b_{\ell^{*}}\right\}\right)>0$. So, $\beta_{2}\left(t_{2},\left\{a_{\infty}\right\}\right)=\beta_{3}\left(t_{3},\left\{b_{\infty}\right\}\right)=1$ must hold indeed.

For every $k \in \mathbf{N}$, let $\sigma^{k}$ be the probability measure on $\left(A_{2}, \mathcal{A}_{2}\right)$ defined by $\sigma^{k}\left(a_{\infty}\right)=0$ and

$$
\sigma^{k}\left(a_{m}\right)=\frac{\beta_{2}^{k}\left(t_{2},\left\{a_{m}\right\}\right)}{1-\beta_{2}^{k}\left(t_{2},\left\{a_{\infty}\right\}\right)}
$$

for every $m \in \mathbf{N}$. So, $\sigma^{k}\left(a_{m}\right)$ equals the probability that action $a_{m}$ is chosen with respect to $\beta_{2}^{k}\left(t_{2}, \cdot\right)$ conditioned on the event that $a_{\infty}$ is not chosen. We now claim that for every $m \in \mathbf{N}$

$$
\lim _{k \rightarrow \infty} \sigma^{k}\left(\left\{a_{1}, \ldots, a_{m}\right\}\right)=0 .
$$

Suppose by way of contradiction that for some $m \in \mathbf{N}$ there exists a $Z>0$ and a subsequence $\left(k_{r}\right)_{r=1}^{\infty}$ such that $\sigma^{k_{r}}\left(\left\{a_{1}, \ldots, a_{m}\right\}\right) \geq Z$ for every $r \in \mathbf{N}$. Notice that, for every $r \in \mathbf{N}$, action $b_{m+1}$ gives in expectation at least $\frac{Z}{m+1}$ to player 3 
against $\sigma^{k_{r}}$, hence at least $\left(1-\beta_{2}^{k_{r}}\left(t_{2},\left\{a_{\infty}\right\}\right)\right) \cdot \frac{Z}{m+1}$ against $\beta^{k_{r}}$. Now consider any action $b_{w}$ with $w>\frac{m+1}{Z}$. Since player 3's highest payoff for action $b_{w}$ is $\frac{1}{w}$, action $b_{w}$ gives player 3 in expectation strictly less than $\frac{Z}{m+1}$ against $\sigma^{k_{r}}$, hence strictly less than $\left(1-\beta_{2}^{k_{r}}\left(t_{2},\left\{a_{\infty}\right\}\right)\right) \cdot \frac{Z}{m+1}$ against $\beta^{k_{r}}$. Therefore, player 3's best responses are in $\left\{b_{1}, \ldots, b_{\left\lfloor\frac{m+1}{Z}\right\rfloor}\right\}$ against $\beta^{k_{r}}$ for any $r \in \mathbf{N}$. Since player 3's best responses along this subsequence are not approaching $b_{\infty}$ with respect to the weak metric, the sequence $\beta^{k}$ cannot satisfy both conditions (1) and (2) of Definition 3.3.3. Thus, (3.3) holds as claimed.

Let $k_{1}=1$ and $m_{1} \in \mathbf{N}$ be such that $\sigma^{k_{1}}\left(\left\{a_{1}, \ldots, a_{m_{1}}\right\}\right)>\frac{1}{2}$. Then, by (3.3), there exists a $k_{2}>k_{1}$ and an $m_{2}>m_{1}$ such that $\sigma^{k_{2}}\left(\left\{a_{m_{1}+1}, \ldots, a_{m_{2}}\right\}\right)>\frac{1}{2}$. By repeating this argument, we obtain in $\mathbf{N}$ two strictly increasing sequences $\left(m_{r}\right)_{r=1}^{\infty}$ and $\left(k_{r}\right)_{r=1}^{\infty}$ such that for every $r \in \mathbf{N}$

$$
\sigma^{k_{r}}\left(\left\{a_{m} \mid m \in W_{r}\right\}\right)>\frac{1}{2},
$$

where $W_{r}=\left\{m_{r-1}+1, \ldots, m_{r}\right\}$ and $m_{0}=0$. Note that the sets $W_{r}, r \in \mathbf{N}$, form a partition of $\mathbf{N}$.

Now let $t_{1}$ be the type in $\{0,1\}^{\mathbf{N}}$ such that $f_{m}\left(t_{1}\right)=1$ if $m \in W_{r}$ for an odd $r \in \mathbf{N}$ and $f_{m}\left(t_{1}\right)=0$ if $m \in W_{r}$ for an even $r \in \mathbf{N}$. Take an arbitrary odd $r \in \mathbf{N}$. Then, $f_{m}\left(t_{1}\right)=1$ for any $m \in W_{r}$, and since $\sigma^{k_{r}}\left(\left\{a_{m} \mid m \in W_{r}\right\}\right)>\frac{1}{2}$ due to (3.4), player 1's unique best response to $\beta^{k_{r}}$ is action $U$. Similarly, when $r \in \mathbf{N}$ is even, player 1's unique best response to $\beta^{k_{r}}$ is action $D$. So, the sequence $\beta^{k}$ cannot satisfy both conditions (1) and (2) of Definition 3.3.3 for type $t_{1}$, which is a contradiction. Consequently, there is no pointwise-perfect strategy profile in this game.

The following corollary follows easily from Proposition 3.3.8.

Corollary 3.3.10 Consider a Bayesian game in which the type spaces are countable, the action spaces are compact, and the payoff functions are continuous. Then, for every a.e.-pointwise-perfect BNE $\beta$, there exists a pointwise-perfect $B N E \hat{\beta}$ such that, for every player $i$, we have $\hat{\beta}_{i}\left(t_{i}, \cdot\right)=\beta_{i}\left(t_{i}, \cdot\right)$ for $\mu_{i}$-a.e. type $t_{i} \in T_{i}$.

Now, we examine Bayesian games which satisfy the following two measurability conditions:

- Condition M1: For any player $i$ and any strategies $\beta_{i}^{1}, \beta_{i}^{2}$ of player $i$, the map

$$
t_{i} \mapsto \rho^{w}\left(\beta_{i}^{1}\left(t_{i}, \cdot\right), \beta_{i}^{2}\left(t_{i}, \cdot\right)\right)
$$

is measurable. 
- Condition M2: For any player $i$, any strategy $\beta_{i}$ of player $i$, and any strategy profile $\tau$, the map

$$
t_{i} \mapsto \rho^{w}\left(\beta_{i}\left(t_{i}, \cdot\right), B R_{i}\left(t_{i}, \tau\right)\right)
$$

is measurable.

Condition M1 is mild, because if the action spaces are $\sigma$-compact, then M1 is always satisfied (cf. Lemma 3.6.2). It is not clear if mild conditions are also sufficient to guarantee M2.

Now, we present a number of conditions that are all equivalent to a.e.-pointwiseperfection, under the additional assumptions of M1 and M2.

Proposition 3.3.11 Consider a Bayesian game which satisfies conditions M1 and M2. Then, for every strategy profile $\beta=\left(\beta_{1}, \ldots, \beta_{n}\right)$, equivalent are:

i. The strategy profile $\beta$ is a.e.-pointwise-perfect.

ii. There exists a sequence of profiles of completely mixed strategies $\left(\beta^{k}\right)_{k=1}^{\infty}=$ $\left(\beta_{1}^{k}, \ldots, \beta_{n}^{k}\right)_{k=1}^{\infty}$ such that for every player $i$ and every $\varepsilon>0$ :

$$
\begin{aligned}
& \text { (ii.1) } \lim _{k \rightarrow \infty} \mu_{i}\left\{t_{i} \in T_{i} \mid \rho^{w}\left(\beta_{i}^{k}\left(t_{i}, \cdot\right), \beta_{i}\left(t_{i}, \cdot\right)\right) \geq \varepsilon\right\}=0, \\
& \text { (ii.2) } \lim _{k \rightarrow \infty} \mu_{i}\left\{t_{i} \in T_{i} \mid \rho^{w}\left(\beta_{i}^{k}\left(t_{i}, \cdot\right), B R_{i}\left(t_{i}, \beta^{k}\right)\right) \geq \varepsilon\right\}=0 .
\end{aligned}
$$

iii. There exists a sequence of profiles of completely mixed strategies $\left(\beta^{k}\right)_{k=1}^{\infty}=$ $\left(\beta_{1}^{k}, \ldots, \beta_{n}^{k}\right)_{k=1}^{\infty}$ such that for every player $i$ :

$$
\begin{aligned}
& \text { (iii.1) } \lim _{k \rightarrow \infty} \int_{T_{i}} \rho^{w}\left(\beta_{i}^{k}\left(t_{i}, \cdot\right), \beta_{i}\left(t_{i}, \cdot\right)\right) \mu_{i}\left(d t_{i}\right)=0, \\
& \text { (iii.2) } \lim _{k \rightarrow \infty} \int_{T_{i}} \rho^{w}\left(\beta_{i}^{k}\left(t_{i}, \cdot\right), B R_{i}\left(t_{i}, \beta^{k}\right)\right) \mu_{i}\left(d t_{i}\right)=0 .
\end{aligned}
$$

The intuition behind conditions (ii) and (iii) is the following. For every player $i$ and every $k \in \mathbf{N}$, define a function $X_{i}^{k}: T_{i} \rightarrow \mathbf{R}$ by $X_{i}^{k}\left(t_{i}\right)=\rho^{w}\left(\beta_{i}^{k}\left(t_{i}, \cdot\right), \beta_{i}\left(t_{i}, \cdot\right)\right)$, and a function $Y_{i}^{k}: T_{i} \rightarrow \mathbf{R}$ by $Y_{i}^{k}\left(t_{i}\right)=\rho^{w}\left(\beta_{i}^{k}\left(t_{i}, \cdot\right), B R_{i}\left(t_{i}, \beta^{k}\right)\right)$. Due to the assumptions $\mathrm{M} 1$ and $\mathrm{M} 2$, these functions are measurable, and therefore they are random variables on the measurable space $\left(T_{i}, \mathcal{T}_{i}\right)$. Conditions (ii.1) and (ii.2) respectively require that both sequences $X_{i}^{k}$ and $Y_{i}^{k}$ converge in probability to zero (i.e. to the random variable that is zero everywhere). So, the probability that $X_{i}^{k}$ and $Y_{i}^{k}$ are far from zero becomes negligible for large $k$. Conditions (iii.1) and (iii.2) mean that both sequences $X_{i}^{k}$ and $Y_{i}^{k}$ converge in expectation to zero, with respect 
to the distribution $\mu_{i}$. This makes intuitive sense because the type of player $i$ is drawn from $T_{i}$ according to $\mu_{i}$.

Proof. The implication (i) $\rightarrow$ (ii) follows immediately from the fact that pointwise convergence almost everywhere implies convergence in probability. The implication (ii) $\rightarrow$ (iii) is also valid, because the sequences $X_{i}^{k}$ and $Y_{i}^{k}$ are uniformly bounded and hence if they converge in probability to zero, then they also converge to zero in expectation. So as to prove the implication (iii) $\rightarrow$ (i), we argue that both implications (iii) $\rightarrow$ (ii) and (ii) $\rightarrow$ (i) are valid. The implication (iii) $\rightarrow$ (ii) holds because convergence in expectation implies convergence in probability. Finally, the implication (ii) $\rightarrow$ (i) follows from the fact that convergence in probability implies the existence of a subsequence that pointwisely converges to the same limit almost everywhere. Hence, if the sequences $X_{i}^{k}$ and $Y_{i}^{k}$ converge in probability to zero, then there are subsequences $X_{i}^{k_{m}}$ and $Y_{i}^{k_{m}}$ which satisfy the conditions of Definition 3.3.6.

The equivalence of the conditions of Proposition 3.3.11 is remarkable in view of the fact that pointwise convergence almost everywhere, convergence in probability and convergence in expectation are not equivalent in general.

\section{Perfection in finite Bayesian games}

In this subsection, we discuss some direct consequences of our previous results on Bayesian games in which there are only finitely many types and actions. A behavioral strategy for player $i$ in such games is a function $\beta_{i}: T_{i} \rightarrow \Delta\left(A_{i}\right)$, where $\Delta\left(A_{i}\right)$ is the set of mixed strategies on $A_{i}$. Behavioral strategy $\beta_{i}$ is called completely mixed if $\beta_{i}\left(t_{i}\right)$ is a completely mixed strategy for each type $t_{i} \in T_{i}$. A direct generalization of Selten's perfect equilibrium to finite Bayesian games is defined as follows:

Definition 3.3.12 A (behavioral) strategy profile $\beta=\left(\beta_{1}, \ldots, \beta_{n}\right)$ is called perfect if there exists a sequence of profiles of completely mixed (behavioral) strategies $\left(\beta^{k}\right)_{k=1}^{\infty}=\left(\beta_{1}^{k}, \ldots, \beta_{n}^{k}\right)_{k=1}^{\infty}$ with the following properties for every player $i$ and each type $t_{i} \in T_{i}$ :

(1) $\lim _{k \rightarrow \infty} d_{E}\left(\beta_{i}^{k}\left(t_{i}\right), \beta_{i}\left(t_{i}\right)\right)=0$,

(2) $\lim _{k \rightarrow \infty} d_{E}\left(\beta_{i}^{k}\left(t_{i}\right), B R_{i}\left(t_{i}, \beta^{k}\right)\right)=0$,

where $d_{E}$ is the Euclidean metric. 
It is clear that the uniform and the pointwise approaches coincide. The a.e.-pointwise approach can however lead to different results, but only in the - perhaps less interesting - case when certain types occur with probability 0 .

Theorem 3.3.13 Every Bayesian game with finitely many types and actions admits a pointwise-perfect (or equivalently, uniform-perfect) $B N E$.

Proof. We only provide a sketch of the proof. First, consider the corresponding game $G^{\prime}$ with ex-ante probabilities in which each player $i$ has action set $A_{i}^{\prime}=\times_{t_{i} \in T_{i}} A_{i}$ and wants to maximize his ex-ante expected payoff, i.e. his expected payoff before he learns his own type. The game $G^{\prime}$ is a game with complete information and with finite action spaces, so it has a perfect equilibrium $\sigma$ in the classical sense with a corresponding sequence of completely mixed strategies $\left(\sigma^{k}\right)_{k=1}^{\infty}$. Let $\beta$ be the (unique) strategy in the original game $G$ such that, given any type $t_{i} \in T_{i}$, the strategies $\sigma$ and $\beta$ induce the same probability on every action $a_{i} \in A_{i}$, i.e. $\beta\left(t_{i}, a_{i}\right)=\sigma\left(A_{i}^{\prime}\left[t_{i}, a_{i}\right]\right)$, where $A_{i}^{\prime}\left[t_{i}, a_{i}\right]$ is the set of all members of $A_{i}^{\prime}$ whose coordinate at position $t_{i}$ is exactly $a_{i}$. Define $\beta^{k}$ for every $k \in \mathbf{N}$ in a similar way with regard to $\sigma^{k}$. It is not difficult to see that $\beta$ is an a.e.-pointwise-perfect BNE, with the sequence $\left(\beta^{k}\right)_{k=1}^{\infty}$ and with $S_{i}$ being for every player $i$ the set of all types $t_{i} \in T_{i}$ that occur with probability 0 . The reason is that if a type $t_{i} \in T_{i}$ occurs with a positive probability, then this type is also taken into account by the ex-ante approach. Now, Proposition 3.3.8 completes the proof.

\subsection{A second-price auction with interdependent values}

We examine perfect equilibrium in a second price auctions with interdependent values, in which bidders have no dominant strategies. We show that perfect equilibrium is a useful tool to select the more natural BNEs in this auction.

Consider the following sealed-bid second price auction $\Gamma^{\alpha}$ for a single indivisible object, where $\alpha$ is a parameter in $(0,1)$. There are two bidders, whose respective types $t_{1}$ and $t_{2}$ are drawn independently from $T_{1}=T_{2}=[0,1]$ according to the uniform distribution. The evaluations of the bidders are symmetric and are given by $v_{1}\left(t_{1}, t_{2}\right)=5+t_{1}-\alpha t_{2}$ and $v_{2}\left(t_{1}, t_{2}\right)=5+t_{2}-\alpha t_{1}$. The set of available bids is $A_{1}=A_{2}=[5-\alpha, 6]$. The tie-breaking rule can be arbitrary, and plays no role in our analysis.

We present different types of BNEs in $\Gamma^{\alpha}$ and show that perfection eliminates the less natural ones. As the proofs in this section are technical in nature, we only discuss the main results, and defer the formal proofs to the appendix. 


\section{A symmetric BNE}

For each bidder $i$, define the pure strategy $B_{i}\left(t_{i}\right)=5+(1-\alpha) t_{i}$, and denote by $\beta_{i}$ the corresponding deterministic strategy.

Proposition 3.4.1 The symmetric deterministic strategy profile $\beta=\left(\beta_{1}, \beta_{2}\right)$ is a $B N E$.

It turns out that $\beta$ is unique within a certain class of strategies. Let $\mathfrak{F}$ be the class of deterministic strategies in which the corresponding pure strategies $p_{i}: T_{i} \rightarrow A_{i}$ for bidders $i=1,2$ satisfy the following conditions:

(i) $p_{1}(0)=p_{2}(0)$ and $p_{1}(1)=p_{2}(1)$,

(ii) $p_{i}$ is differentiable and $\frac{d}{d t_{i}} p_{i}\left(t_{i}\right)>0$ for every $t_{i} \in T_{i}$.

Now, we have the following proposition:

Proposition 3.4.2 The BNE $\beta$ is the only pure BNE in class $\mathfrak{F}$.

Proposition 3.4.3 The BNE $\beta$ is not sincere in the sense that for each bidder $i$, $B_{i}\left(t_{i}\right)$ does not equal his expected evaluation of the object given $t_{i}$, except for $t_{i}=$ $\frac{1}{2}$.

Proposition 3.4.4 Neither bidder has a dominant strategy in the auction $\Gamma^{\alpha}$ in the following sense: neither bidder $i$ has a strategy $\beta_{i}$ such that for every type $t_{i}$, every strategy $\sigma_{i}$ of bidder $i$ and every strategy $\beta_{-i}$ of bidder $i$ 's opponents we have

$$
\boldsymbol{E}_{\beta_{-i}}\left(\Pi_{i} \mid t_{i}, \beta_{i}\right) \geq \boldsymbol{E}_{\beta_{-i}}\left(\Pi_{i} \mid t_{i}, \sigma_{i}\right) .
$$

In fact we will prove a stronger statement. Namely, in the above statement we could replace "every type $t_{i}$ " by "every type $t_{i}$ in a subset of types with a strictly positive measure". In view of the previous proposition, dominance is not a useful criterion to select BNEs in this auction. Nevertheless, as we will show, perfection can be used with great effect.

Proposition 3.4.5 The BNE $\beta$ is uniform-perfect.

We will now describe the idea of the proof. Let $C_{M}:[0,1] \rightarrow[5-\alpha, 6]$ be defined by $C_{M}(r)=5+(1-\alpha) r$ for all $r \in[0,1]$ and $\mu$ be the uniform distribution on $[5-\alpha, 6]$. The first idea would be to look at the sequences of completely mixed strategies $\hat{\beta}_{i}^{k}$ given by $\hat{\beta}_{i}^{k}\left(t_{i}, \cdot\right)=\left(1-\varepsilon_{k}\right) \delta_{C_{M}\left(t_{i}\right)}(\cdot)+\varepsilon_{k} \mu(\cdot)$ for both players $i=1,2$ and for every $t_{i}$, where $\delta$ is the Dirac measure and $\varepsilon_{k}$ is a sequence in $(0,1)$ 
converging to zero. One can verify that the sequence $\hat{\beta}_{i}^{k}$ satisfies condition (1) of Definition 3.3.1, and $\lim _{k \rightarrow \infty} \rho^{w}\left(\hat{\beta}_{i}^{k}\left(t_{i}, \cdot\right), B R_{i}\left(t_{i}, \hat{\beta}^{k}\right)\right)=0$ for every $t_{i} \in(0,1)$. However, the latter does not hold for $t_{i}=0,1$. More precisely, one can easily check that $B R_{i}\left(0, \hat{\beta}^{k}\right)=\left\{5-\frac{1}{2} \alpha\right\}$ and $B R_{i}\left(1, \hat{\beta}^{k}\right)=\left\{6-\frac{1}{2} \alpha\right\}$, for every $k$. However, by defining the completely mixed strategies in a more delicate way, we can prove that $\left(\beta_{1}, \beta_{2}\right)$ is uniform-perfect. We introduce two more curves $C_{T}, C_{L}:[0,1] \rightarrow$ $[5-\alpha, 6]$ by $C_{T}(r)=6-\alpha r^{2}$, and $C_{L}(r)=5-\alpha+\alpha(1-r)^{2}$. These curves are depicted in the picture below.

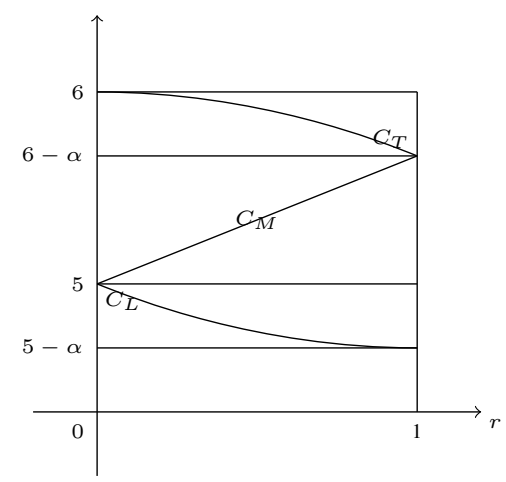

The curves $C_{T}$ and $C_{L}$ will be used to repair the above mentioned problem for types 0 and 1 . For every $k \in \mathbf{N}$, let $\varepsilon_{k}=\frac{1}{k+3}$ and $\beta_{1}^{k}: T_{1} \times \mathcal{A}_{1} \rightarrow[0,1]$ be the completely mixed strategy for bidder 1 which is given for every $t_{1} \in T_{1}=[0,1]$ by

$$
\beta_{1}^{k}\left(t_{1}, \cdot\right)=\left(1-2 \varepsilon_{k}-\varepsilon_{k}^{2}\right) \delta_{C_{M}\left(t_{1}\right)}(\cdot)+\varepsilon_{k} \delta_{C_{T}\left(t_{1}\right)}(\cdot)+\varepsilon_{k} \delta_{C_{L}\left(t_{1}\right)}(\cdot)+\varepsilon_{k}^{2} \mu(\cdot) .
$$

The cumulative probability distribution with respect to $\beta_{1}^{k}\left(t_{1}, \cdot\right)$ is shown on $A_{1}=[5-\alpha, 6]$ in the picture below.

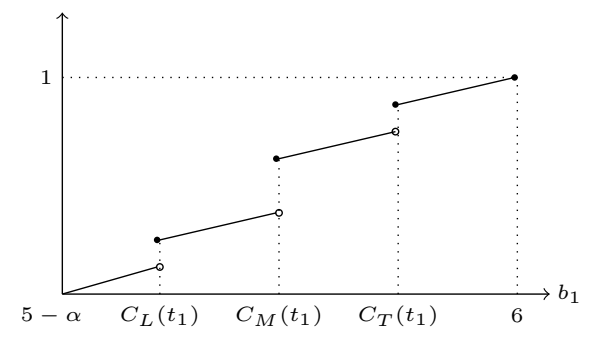

Similarly, define a completely mixed strategy $\beta_{2}^{k}$ for bidder 2 . We will show that the sequence of strategy profiles $\left(\beta^{k}\right)_{k=1}^{\infty}=\left(\beta_{1}^{k}, \beta_{2}^{k}\right)_{k=1}^{\infty}$ satisfies conditions 1 and 2 of Definition 3.3.1. 


\section{Other BNEs}

In this subsection, we present other BNEs for the auction. They are arguably less intuitive and, as we show, perfection eliminates all of them. We start with Wolf and Sheep BNEs.

Proposition 3.4.6 The deterministic strategy profile $\sigma=\left(\sigma_{1}, \sigma_{2}\right)$, given by $\sigma_{1}\left(t_{1}, \cdot\right)=$ $\delta_{6}(\cdot)$ for every $t_{1} \in T_{1}$ and $\sigma_{2}\left(t_{2}, \cdot\right)=\delta_{5-\alpha}(\cdot)$ for every $t_{2} \in T_{2}$, is a BNE. However, $\sigma$ is not pointwise-perfect, and hence not uniform-perfect either.

It is worthwhile to note that the BNE introduced in Proposition 3.4.6 is just one of the many other BNEs of the same type. In all of this type of BNEs, the lowest bid of Wolf player is strictly larger than the highest bid of Sheep player.

The following proposition considers a deterministic BNE in which the corresponding pure strategies, as functions of the types, are not continuous.

Proposition 3.4.7 The deterministic strategy profile $\eta=\left(\eta_{1}, \eta_{2}\right)$ is a BNE, where $\eta_{1}$ and $\eta_{2}$ correspond to the pure strategies $b_{1}$ and $b_{2}$ given by

$$
\begin{aligned}
& b_{1}\left(t_{1}\right)=\left\{\begin{array}{ll}
5 & \text { if } t_{1} \in[0, x(\alpha)] \\
6 & \text { if } t_{1} \in(x(\alpha), 1]
\end{array} \text { and } b_{2}\left(t_{2}\right)=\left\{\begin{array}{ll}
5-\alpha & \text { if } t_{2} \in[0, y(\alpha)] \\
5.1 & \text { if } t_{2} \in(y(\alpha), 1]
\end{array},\right.\right. \\
& \text { with } x(\alpha)=\frac{0.4+2 \alpha}{4-\alpha^{2}} \text { and } y(\alpha)=\frac{\alpha(0.2+\alpha)}{4-\alpha^{2}} \text {. }
\end{aligned}
$$

However, $\eta$ is not pointwise-perfect, and hence not uniform-perfect either.

\subsection{Appendix I: Proofs for the auction in Section 3.4}

Throughout the Appendix.2, we will use the notion $\mathbf{E}_{\tau_{1}}\left(\Pi_{2} \mid t_{2}, b_{2}\right)$ for bidder 2's expected profit given his type $t_{2}$ and bid $b_{2}$ against a strategy $\tau_{1}$ of bidder 1 . If bidder 1 makes a bid $b_{1}>b_{2}$, then bidder 2 does not win the object and has zero profit. Therefore, if bidding $b_{2}$ against $\tau_{1}$ leads to a tie with zero ex-ante probability, then we have

$$
\mathbf{E}_{\tau_{1}}\left(\Pi_{2} \mid t_{2}, b_{2}\right)=\int_{0}^{1} \int_{5-\alpha}^{b_{2}}\left(5+t_{2}-\alpha t_{1}-b_{1}\right) \tau_{1}\left(t_{1}, d b_{1}\right) d t_{1} .
$$

Since the function $5+t_{2}-\alpha t_{1}-b_{1}$ is bounded on $[0,1] \times[5-\alpha, 6]$ and measurable with respect to $\mathcal{T} \times \mathcal{A}$, then according to Theorem $4.6 .5, \mathbf{E}_{\tau_{1}}\left(\Pi_{2} \mid t_{2}, b_{2}\right)$ is welldefined.

In the special case when $\tau_{1}$ is a deterministic strategy, i.e. when $\tau_{1}\left(t_{1}, \cdot\right)=$ $\delta_{p_{1}\left(t_{1}\right)}(\cdot)$ for a pure strategy $p_{1}$, we have

$$
\int_{5-\alpha}^{b_{2}}\left(5+t_{2}-\alpha t_{1}-b_{1}\right) \tau_{1}\left(t_{1}, d b_{1}\right)=
$$




$$
\begin{cases}5+t_{2}-\alpha t_{1}-p_{1}\left(t_{1}\right) & \text { if } p_{1}\left(t_{1}\right) \in\left[5-\alpha, b_{2}\right] \\ 0 & \text { if } p_{1}\left(t_{1}\right) \in\left(b_{2}, 6\right]\end{cases}
$$

Hence, if $p_{1}$ is increasing and $b_{2} \in p_{1}\left(T_{1}\right)$, then we have

$$
\mathbf{E}_{\tau_{1}}\left(\Pi_{2} \mid t_{2}, b_{2}\right)=\int_{0}^{p_{1}^{-1}\left(b_{2}\right)}\left(5+t_{2}-\alpha t_{1}-p_{1}\left(t_{1}\right)\right) d t_{1} .
$$

\section{Proof of Proposition 3.4.1}

We prove that the deterministic strategy profile $\beta=\left(\beta_{1}, \beta_{2}\right)$ in which $\beta_{i}$ corresponds to the pure strategy $B_{i}\left(t_{i}\right)=5+(1-\alpha) t_{i}$ for each bidder $i$, is a BNE. For this purpose, we prove that $\beta_{2}$ is a best response against $\beta_{1}$. Then, due to symmetry, $\beta_{1}$ is also a best response against $\beta_{2}$, and the proof will be complete.

According to (3.6), if $b_{2} \in[5,6-\alpha]$ then we have

$$
\begin{aligned}
\mathbf{E}_{\beta_{1}}\left(\Pi_{2} \mid t_{2}, b_{2}\right) & =\int_{0}^{B_{1}^{-1}\left(b_{2}\right)}\left(5+t_{2}-\alpha t_{1}-B_{1}\left(t_{1}\right)\right) d t_{1} \\
& =\int_{0}^{\frac{b_{2}-5}{1-\alpha}}\left(5+t_{2}-\alpha t_{1}-5-(1-\alpha) t_{1}\right) d t_{1} \\
& =\int_{0}^{\frac{b_{2}-5}{1-\alpha}}\left(t_{2}-t_{1}\right) d t_{1} \\
& =t_{2}\left(\frac{b_{2}-5}{1-\alpha}\right)-\frac{1}{2}\left(\frac{b_{2}-5}{1-\alpha}\right)^{2} .
\end{aligned}
$$

By taking the first derivative with respect to $b_{2}$

$$
\frac{d}{d b_{2}} \mathbf{E}_{\beta_{1}}\left(\Pi_{2} \mid t_{2}, b_{2}\right)=\frac{t_{2}}{1-\alpha}-\frac{1}{1-\alpha}\left(\frac{b_{2}-5}{1-\alpha}\right) .
$$

It is clear that

$$
\frac{d}{d b_{2}} \mathbf{E}_{\beta_{1}}\left(\Pi_{2} \mid t_{2}, 5\right) \geq 0 \text { and } \frac{d}{d b_{2}} \mathbf{E}_{\beta_{1}}\left(\Pi_{2} \mid t_{2}, 6-\alpha\right) \leq 0,
$$

which implies that the maximum of $\mathbf{E}_{\beta_{1}}\left(\Pi_{2} \mid t_{2}, b_{2}\right)$ over $b_{2} \in[5,6-\alpha]$ is attained where $\frac{d}{d b_{2}} \mathbf{E}_{\beta_{1}}\left(\Pi_{2} \mid t_{2}, b_{2}\right)=0$. This happens exactly when

$$
b_{2}=5+(1-\alpha) t_{2}=B_{2}\left(t_{2}\right) .
$$

Notice that, for bidder 2, bidding less than 5 is never better than bidding 5 exactly, because all bids less than or equal to 5 win against $\beta_{1}$ with probability 0 . Similarly, bidding more than $6-\alpha$ is never better than bidding $6-\alpha$ exactly, because all bids larger than or equal to $6-\alpha$ win against $\beta_{1}$ with probability 1 . Therefore, $\beta_{2}$ is a best response to $\beta_{1}$ as claimed. 


\section{Proof of Proposition 3.4.2}

Consider two pure strategies $p_{1}$ and $p_{2}$ that satisfy conditions (i), (ii) of Proposition 3.4.2, and the corresponding deterministic strategies $\tau_{1}$ and $\tau_{2}$. Suppose that $\left(\tau_{1}, \tau_{2}\right)$ is a BNE. Our goal is to show that $p_{1}\left(t_{1}\right)=5+(1-\alpha) t_{1}$ and $p_{2}\left(t_{2}\right)=5+(1-\alpha) t_{2}$.

Notice that $p_{1}: T_{1} \rightarrow A_{1}$ and $p_{2}: T_{2} \rightarrow A_{2}$ are continuous and invertible, and therefore they have a continuous inverse. Thus, because $p_{1}\left(T_{1}\right)=p_{2}\left(T_{2}\right)$, the function $\hat{p}=p_{1}^{-1} \circ p_{2}$ is well defined and it is a continuous bijection from $T_{2}$ to $T_{1}$.

First we argue that $p_{2}(0) \leq 5$. Suppose by way of contradiction that $p_{2}(0)>5$. According to (3.6) we have

$$
\mathbf{E}_{\tau_{1}}\left(\Pi_{2} \mid t_{2}, p_{2}\left(t_{2}\right)\right)=\int_{0}^{\hat{p}\left(t_{2}\right)}\left(5+t_{2}-\alpha t_{1}-p_{1}\left(t_{1}\right)\right) d t_{1} .
$$

Notice that if $t_{2}>0$ then $\hat{p}\left(t_{2}\right)>0$ due to conditions (i) and (ii), and moreover, for every type $t_{2}<p_{2}(0)-5$ we have

$$
5+t_{2}-\alpha t_{1}-p_{1}\left(t_{1}\right) \leq 5+t_{2}-p_{1}\left(t_{1}\right) \leq 5+t_{2}-p_{1}(0)=5+t_{2}-p_{2}(0)<0 .
$$

Therefore, $\mathbf{E}_{\tau_{1}}\left(\Pi_{2} \mid t_{2}, p_{2}\left(t_{2}\right)\right)$ is strictly negative. However, as $p_{1}(0)=p_{2}(0)>$ 5 , bidder 2 can get zero by bidding 5 for instance, which never wins. This is a contradiction with the assumption that $\tau_{2}$ is a best response to $\tau_{1}$, so $p_{2}(0) \leq 5$ holds indeed.

Now we prove that $p_{2}(1) \geq 6-\alpha$. Write $c:=6-\alpha-p_{2}(1)$ and suppose by way of contradiction that $c>0$. According to (3.6), if we compare bids $p_{2}(1)$ and $p_{2}\left(t_{2}\right)$ for bidder 2 when his type is $t_{2}$, we obtain

$$
\mathbf{E}_{\tau_{1}}\left(\Pi_{2} \mid t_{2}, p_{2}(1)\right)-\mathbf{E}_{\tau_{1}}\left(\Pi_{2} \mid t_{2}, p_{2}\left(t_{2}\right)\right)=\int_{\hat{p}\left(t_{2}\right)}^{1}\left(5+t_{2}-\alpha t_{1}-p_{1}\left(t_{1}\right)\right) d t_{1} .
$$

Notice that if $t_{2} \in(1-c, 1)$, then $\hat{p}\left(t_{2}\right)<1$ due to conditions (i) and (ii), and moreover, for every $t_{1} \in[0,1]$ we have

$$
\begin{aligned}
5+t_{2}-\alpha t_{1}-p_{1}\left(t_{1}\right) & \geq 5+t_{2}-\alpha-p_{1}(1) \\
& =5+t_{2}-\alpha-p_{2}(1) \\
& =5+t_{2}-\alpha+c-6+\alpha \\
& =-1+t_{2}+c \\
& >0 .
\end{aligned}
$$

Therefore, for every $t_{2} \in(1-c, 1)$

$$
\mathbf{E}_{\tau_{1}}\left(\Pi_{2} \mid t_{2}, p_{2}(1)\right)-\mathbf{E}_{\tau_{1}}\left(\Pi_{2} \mid t_{2}, p_{2}\left(t_{2}\right)\right)>0,
$$


which is in contradiction with the assumption that $\tau_{2}$ is a best response to $\tau_{1}$. Hence, $p_{2}(1) \geq 6-\alpha$ holds indeed.

Now, we calculate bidder 2's best response bids against $\tau_{1}$, given his type $t_{2}$. It is clear that, for any type $t_{2}$, bidding $p_{2}(0)$ is not worse than any bid in $\left[5-\alpha, p_{2}(0)\right]$, because all these bids win with probability zero against $\tau_{1}$. Similarly, bidding $p_{2}(1)$ is not worse than any bid in $\left[p_{2}(1), 6\right]$, because all these bids win with probability 1 against $\tau_{1}$. It remains to determine the best bids in the interval $\left[p_{2}(0), p_{2}(1)\right]$, which we do by examining the derivative of $\mathbf{E}_{\tau_{1}}\left(\Pi_{2} \mid t_{2}, b_{2}\right)$ with respect to $b_{2}$. By (3.6), we have for every bid $b_{2} \in\left[p_{2}(0), p_{2}(1)\right]$ that

$$
\begin{aligned}
\mathbf{E}_{\tau_{1}}\left(\Pi_{2} \mid t_{2}, b_{2}\right) & =\int_{0}^{p_{1}^{-1}\left(b_{2}\right)}\left(5+t_{2}-\alpha t_{1}-p_{1}\left(t_{1}\right)\right) d t_{1} \\
& =\left[\left(5+t_{2}\right) t_{1}-\frac{\alpha}{2} t_{1}^{2}\right]_{0}^{p_{1}^{-1}\left(b_{2}\right)}-\int_{0}^{p_{1}^{-1}\left(b_{2}\right)} p_{1}\left(t_{1}\right) d t_{1} \\
& =\left(5+t_{2}\right) p_{1}^{-1}\left(b_{2}\right)-\frac{\alpha}{2}\left(p_{1}^{-1}\left(b_{2}\right)\right)^{2}-\int_{0}^{p_{1}^{-1}\left(b_{2}\right)} p_{1}\left(t_{1}\right) d t_{1} .
\end{aligned}
$$

Therefore, the first derivative with respect to $b_{2}$ is

$$
\begin{aligned}
\frac{d}{d b_{2}} \mathbf{E}_{\tau_{1}}\left(\Pi_{2} \mid t_{2}, b_{2}\right) & =\frac{1}{p_{1}^{\prime}\left(p_{1}^{-1}\left(b_{2}\right)\right)} \cdot\left(5+t_{2}-\alpha p_{1}^{-1}\left(b_{2}\right)\right) \\
& -\frac{1}{p_{1}^{\prime}\left(p_{1}^{-1}\left(b_{2}\right)\right)} p_{1}\left(p_{1}^{-1}\left(b_{2}\right)\right) \\
& =\frac{1}{p_{1}^{\prime}\left(p_{1}^{-1}\left(b_{2}\right)\right)} \cdot\left(5+t_{2}-\alpha p_{1}^{-1}\left(b_{2}\right)-b_{2}\right) .
\end{aligned}
$$

Because $\hat{p}(0)=p_{1}^{-1}\left(p_{2}(0)\right)=p_{1}^{-1}\left(p_{1}(0)\right)=0$ and $p_{1}^{\prime}\left(p_{1}^{-1}\left(b_{2}\right)\right)>0$ by conditions (i) and (ii), and because $p_{2}(0) \leq 5$ we have for every $t_{2}>0$ that

$$
\frac{d}{d b_{2}} \mathbf{E}_{\tau_{1}}\left(\Pi_{2} \mid t_{2}, p_{2}(0)\right)>0 .
$$

Similarly, because $\hat{p}(1)=p_{1}^{-1}\left(p_{2}(1)\right)=p_{1}^{-1}\left(p_{1}(1)\right)=1$ and $p_{1}^{\prime}\left(p_{1}^{-1}\left(b_{2}\right)\right)>0$ by conditions (i) and (ii), and because $p_{2}(1) \geq 6-\alpha$ we have for every $t_{2}<1$ that

$$
\frac{d}{d b_{2}} \mathbf{E}_{\tau_{1}}\left(\Pi_{2} \mid t_{2}, p_{2}(1)\right)<0
$$

In view of (3.7) and (3.8), for all $t_{2} \in(0,1)$, each best response bid $b_{2}$ in $\left[p_{2}(0), p_{2}(1)\right]$ has to satisfy

$$
\frac{d}{d b_{2}} \mathbf{E}_{\tau_{1}}\left(\Pi_{2} \mid t_{2}, b_{2}\right)=0
$$


which is equivalent to

$$
b_{2}=5+t_{2}-\alpha p_{1}^{-1}\left(b_{2}\right) .
$$

Because $\tau_{2}$ is a best response against $\tau_{1}$, we must have for every $t_{2} \in(0,1)$ that

$$
p_{2}\left(t_{2}\right)=5+t_{2}-\alpha p_{1}^{-1}\left(p_{2}\left(t_{2}\right)\right)=5+t_{2}-\alpha \hat{p}\left(t_{2}\right) .
$$

By continuity of $p_{2}$ and $\hat{p}$, we obtain for all $t_{2} \in[0,1]$ that

$$
p_{2}\left(t_{2}\right)=5+t_{2}-\alpha \hat{p}\left(t_{2}\right) .
$$

With a similar argument for bidder 1 , we have for all $t_{1} \in[0,1]$ that

$$
p_{1}\left(t_{1}\right)=5+t_{1}-\alpha \hat{p}^{-1}\left(t_{1}\right) .
$$

By equation (3.10) we have for all $t_{2} \in[0,1]$ that

$$
p_{1}\left(\hat{p}\left(t_{2}\right)\right)=5+\hat{p}\left(t_{2}\right)-\alpha \hat{p}^{-1}\left(\hat{p}\left(t_{2}\right)\right),
$$

which yields $p_{2}\left(t_{2}\right)=5+\hat{p}\left(t_{2}\right)-\alpha t_{2}$. Thus, by equation (3.9) we have

$$
5+\hat{p}\left(t_{2}\right)-\alpha t_{2}=5+t_{2}-\alpha \hat{p}\left(t_{2}\right),
$$

which implies $\hat{p}\left(t_{2}\right)=t_{2}$. Hence, from (3.9) we derive that

$$
p_{2}\left(t_{2}\right)=5+(1-\alpha) t_{2},
$$

and because of symmetry also that

$$
p_{1}\left(t_{1}\right)=5+(1-\alpha) t_{1},
$$

so the proof of Proposition 3.4.2 is complete.

\section{Proof of Proposition 3.4.3}

Due to symmetry, it suffices to prove the statement only for bidder 1 . Notice that bidder 1's expected evaluation of the object given his type $t_{1}$ is

$$
\mathbf{E}\left(v_{1}\left(t_{1}, t_{2}\right) \mid t_{1}\right)=\mathbf{E}\left(5+t_{1}-\alpha t_{2} \mid t_{1}\right)=5+t_{1}-\frac{\alpha}{2},
$$

which differs from $B_{1}\left(t_{1}\right)=5+(1-\alpha) t_{1}$ for every type $t_{1} \in[0,1] \backslash\left\{\frac{1}{2}\right\}$, as claimed. It is worthwhile to mention that even if we look at the situation in which bidder 1 knows that he is winning, still his expected evaluation of the object given his type $t_{1}$ is not equal to his bid almost everywhere :

$$
\begin{aligned}
\mathbf{E}\left(v_{1}\left(t_{1}, t_{2}\right) \mid b_{1}\left(t_{1}\right)>b_{2}\left(t_{2}\right)\right) & =\mathbf{E}\left(5+t_{1}-\alpha t_{2} \mid t_{1}>t_{2}\right) \\
& =5+t_{1}-\alpha \mathbf{E}\left(t_{2} \mid t_{1}>t_{2}\right)=5+t_{1}-\frac{\alpha}{2} t_{1},
\end{aligned}
$$

which is strictly larger than $B_{1}\left(t_{1}\right)=5+(1-\alpha) t_{1}$ for every type $t_{1} \in(0,1]$. 


\section{Proof of Proposition 3.4.4}

We prove that neither bidder has a dominant strategy. To this end we prove that every strategy $\sigma_{i}$ of player $i=1,2$ is not dominant. Suppose $\sigma_{2}$ is a dominant strategy for bidder 2 . Notice that if bidder 1 chooses strategy $\beta_{1}$ and if bidder 2 is given type $t_{2} \in(0,1)$, then bidding $\beta_{2}$ is bidder 2's unique best response (see the proof of Proposition 3.4.1). This implies that $\sigma_{2}=\beta_{2}$. Now we show that the strategies $\beta_{2}$ is not in dominant strategies. To this end we show that $\beta_{2}$ is not a best response against all strategies of bidder 1. Suppose that bidder 1's strategy is given by $\lambda_{1}\left(t_{1}, \cdot\right)=\delta_{b_{1}\left(t_{1}\right)}(\cdot)$ for every $t_{1} \in T_{1}$, where

$$
b_{1}\left(t_{1}\right)= \begin{cases}\frac{11}{2} & \text { if } t_{1} \in\left[0, \frac{1}{2}\right] \\ 6 & \text { if } t_{1} \in\left(\frac{1}{2}, 1\right]\end{cases}
$$

We prove that if $t_{2}=\frac{1+\alpha}{2}$, then $\beta_{2}$ is not a best response for bidder 2 against $\lambda_{1}$. Notice that bidder 2 gets zero by bidding $B_{2}\left(\frac{1+\alpha}{2}\right)$, because it never wins against $\lambda_{1}$ due to $B_{2}\left(\frac{1+\alpha}{2}\right)=5+(1-\alpha)\left(\frac{1+\alpha}{2}\right)<\frac{11}{2}$. However, by (3.6), bidder 2's expected profit given type $t_{2}=\frac{1+\alpha}{2}$ and $b_{2} \in\left(\frac{11}{2}, 6\right)$ is

$$
\mathbf{E}_{\lambda_{1}}\left(\Pi_{2} \mid \frac{1+\alpha}{2}, b_{2}\right)=\int_{0}^{\frac{1}{2}}\left(5+\frac{1+\alpha}{2}-\alpha t_{1}-\frac{11}{2}\right) d t_{1}=\frac{\alpha}{8}>0 .
$$

This shows that if $t_{2}=\frac{1+\alpha}{2}$ then bidding $b_{2} \in\left(\frac{11}{2}, 6\right)$ is strictly better than bidding $B_{2}\left(\frac{1+\alpha}{2}\right)$. Hence, $\beta_{2}$ is not in dominant strategies. This proves that bidder 2 does not have a dominant strategy at all, and by symmetry the same is true for bidder 1 . The proof is complete.

\section{Proof of Proposition 3.4.5}

We prove that the $\mathrm{BNE} \beta=\left(\beta_{1}, \beta_{2}\right)$ is uniform-perfect. For this purpose, define the curves $C_{M}, C_{T}, C_{L}:[0,1] \rightarrow[5-\alpha, 6]$ by

$$
\begin{gathered}
C_{M}\left(t_{1}\right)=5+(1-\alpha) t_{1}, \\
C_{T}\left(t_{1}\right)=6-\alpha t_{1}^{2}, \\
C_{L}\left(t_{1}\right)=5-\alpha+\alpha\left(1-t_{1}\right)^{2} .
\end{gathered}
$$

Let $\mu$ be the uniform distribution on $A_{1}=[5-\alpha, 6]$. For every $k \in \mathbf{N}$, let $\varepsilon_{k}=\frac{1}{k+3}$ and $\beta_{1}^{k}: T_{1} \times \mathcal{A}_{1} \rightarrow[0,1]$ be the completely mixed strategy for bidder 1 which is given for every $t_{1} \in T_{1}=[0,1]$ by

$$
\beta_{1}^{k}\left(t_{1}, \cdot\right)=\left(1-2 \varepsilon_{k}-\varepsilon_{k}^{2}\right) \delta_{C_{M}\left(t_{1}\right)}(\cdot)+\varepsilon_{k} \delta_{C_{T}\left(t_{1}\right)}(\cdot)+\varepsilon_{k} \delta_{C_{L}\left(t_{1}\right)}(\cdot)+\varepsilon_{k}^{2} \mu(\cdot) .
$$


Similarly, define a completely mixed strategy $\beta_{2}^{k}$ for bidder 2 . We show that the sequence of strategy profiles $\left(\beta^{k}\right)_{k=1}^{\infty}=\left(\beta_{1}^{k}, \beta_{2}^{k}\right)_{k=1}^{\infty}$ satisfies conditions 1 and 2 of Definition 3.3.1.

It is clear from the definition of $\beta_{i}\left(t_{i}, \cdot\right)$ and $\beta_{i}^{k}\left(t_{i}, \cdot\right)$, for each bidder $i$, that

$$
\sup _{t_{i} \in T_{i}} \rho^{w}\left(\beta_{i}^{k}\left(t_{i}, \cdot\right), \beta_{i}\left(t_{i}, \cdot\right)\right)=2 \varepsilon_{k}+\varepsilon_{k}^{2}
$$

Hence, condition 1 of Definition 3.3.1 is satisfied. Condition 2 of Definition 3.3.1 follows from the claim below.

Claim: For every $\xi \in(0, \alpha)$, there is a $K_{\xi} \in \mathbf{N}$ such that for every $k>K_{\xi}$ we have:

1. For every $t_{2} \in[0, \xi)$, the maximum of $\mathbf{E}_{\beta_{1}^{k}}\left(\Pi_{2} \mid t_{2}, b_{2}\right)$ over $b_{2} \in[5-\alpha, 6]$ can only be attained within $b_{2} \in[5-\xi, 5+2 \xi(1-\alpha)]$.

2. For every $t_{2} \in[\xi, 1-\xi]$, the maximum of $\mathbf{E}_{\beta_{1}^{k}}\left(\Pi_{2} \mid t_{2}, b_{2}\right)$ over $b_{2} \in[5-\alpha, 6]$ can only be attained within $b_{2} \in\left[C_{M}\left(t_{2}\right)-\xi(1-\alpha), C_{M}\left(t_{2}\right)+\xi(1-\alpha)\right]$.

3. For every $t_{2} \in(1-\xi, 1]$ the maximum of $\mathbf{E}_{\beta_{1}^{k}}\left(\Pi_{2} \mid t_{2}, b_{2}\right)$ over $b_{2} \in[5-\alpha, 6]$ can only be attained within $b_{2} \in[6-\alpha-2 \xi(1-\alpha), 6-\alpha+\xi]$.

The above claim is illustrated in Figure 3.1. According to the claim, the gray area includes all best response bids for bidder 2 if $k>K_{\xi}$.

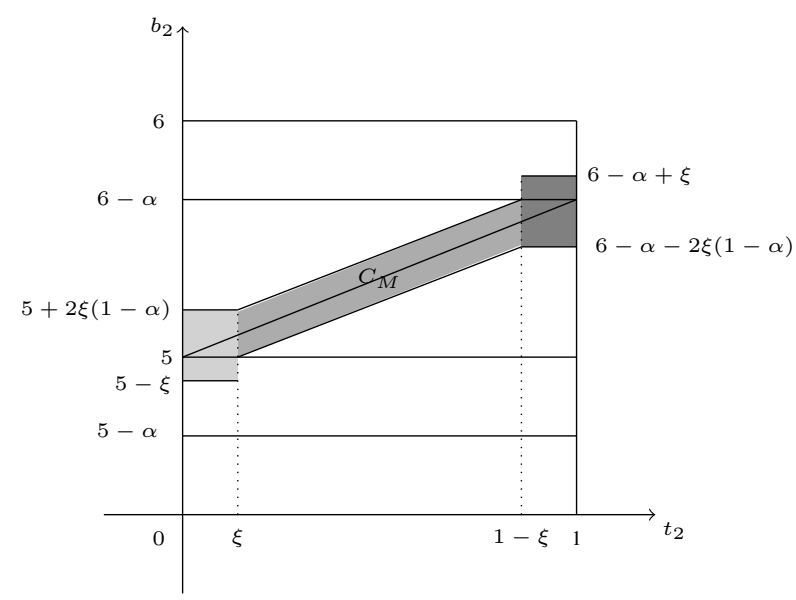

Figure 3.1: bidder 2's best response bids 
Take an arbitrary $\xi \in\left(0, \min \left\{\alpha, \frac{1}{2}\right\}\right)$. To prove this claim, it is enough to show that the following claims 1,2 and 3 are valid. These claims corresponds to three cases, i.e., $6-\alpha \leq b_{2} \leq 6,5-\alpha \leq b_{2} \leq 5$ and $5 \leq b_{2} \leq 6-\alpha$.

Claim 1: for the case $6-\alpha \leq b_{2} \leq 6$

There is an $M_{\xi}^{1} \in \mathbf{N}$ such that for every $k>M_{\xi}^{1}$ we have:

1. For every $t_{2} \in[0,1-\xi]$, the maximum of $\mathbf{E}_{\beta_{1}^{k}}\left(\Pi_{2} \mid t_{2}, b_{2}\right)$ over $b_{2} \in[6-\alpha, 6]$ is attained at $b_{2}=6-\alpha$.

2. For every $t_{2} \in(1-\xi, 1]$, the maximum of $\mathbf{E}_{\beta_{1}^{k}}\left(\Pi_{2} \mid t_{2}, b_{2}\right)$ over $b_{2} \in[6-\alpha, 6]$ can only be attained within $b_{2} \in[6-\alpha, 6-\alpha+\xi]$.

Claim 2: for the case $5-\alpha \leq b_{2} \leq 5$

There is an $M_{\xi}^{2} \in \mathbf{N}$ such that for every $k>M_{\xi}^{2}$ we have:

1. For every $t_{2} \in[\xi, 1]$, the maximum of $\mathbf{E}_{\beta_{1}^{k}}\left(\Pi_{2} \mid t_{2}, b_{2}\right)$ over $b_{2} \in[5-\alpha, 5]$ is attained at $b_{2}=5$.

2. For every $t_{2} \in[0, \xi)$, the maximum of $\mathbf{E}_{\beta_{1}^{k}}\left(\Pi_{2} \mid t_{2}, b_{2}\right)$ over $b_{2} \in[5-\alpha, 5]$ can only be attained within $b_{2} \in[5-\xi, 5]$.

Claim 3: for case $5 \leq b_{2} \leq 6-\alpha$

There is an $M_{\xi}^{3} \in \mathbf{N}$ such that for every $k>M_{\xi}^{3}$ we have:

1. For every $t_{2} \in[\xi, 1-\xi]$, the maximum of $\mathbf{E}_{\beta_{1}^{k}}\left(\Pi_{2} \mid t_{2}, b_{2}\right)$ over $b_{2} \in[5,6-\alpha]$ can only be attained within $b_{2} \in\left[C_{M}\left(t_{2}\right)-\xi(1-\alpha), C_{M}\left(t_{2}\right)+\xi(1-\alpha)\right]$.

2. For every $t_{2} \in[0, \xi)$, the maximum of $\mathbf{E}_{\beta_{1}^{k}}\left(\Pi_{2} \mid t_{2}, b_{2}\right)$ over $b_{2} \in[5,6-\alpha]$ can only be attained within $b_{2} \in[5,5+2 \xi(1-\alpha)]$.

3. For every $t_{2} \in(1-\xi, 1]$ the maximum of $\mathbf{E}_{\beta_{1}^{k}}\left(\Pi_{2} \mid t_{2}, b_{2}\right)$ over $b_{2} \in[5,6-\alpha]$ can only be attained within $b_{2} \in[6-\alpha-2 \xi(1-\alpha), 6-\alpha]$.

It is important to note that Part (1) of Claim 1 shows that for every $t_{2} \in[0,1-\xi]$, the maximum of $\mathbf{E}_{\beta_{1}^{k}}\left(\Pi_{2} \mid t_{2}, b_{2}\right)$ over $b_{2} \in[6-\alpha, 6]$ is not more than the maximum over $b_{2} \in[5,6-\alpha]$, which is verified in Claim 3. Similarly, Part (1) of Claim 2 indicates that for every $t_{2} \in[\xi, 1]$, the maximum of $\mathbf{E}_{\beta_{1}^{k}}\left(\Pi_{2} \mid t_{2}, b_{2}\right)$ over $b_{2} \in$ $[5-\alpha, 5]$ is not more than the maximum over $b_{2} \in[5,6-\alpha]$.

Proof of Claim 1: We compute $\mathbf{E}_{\beta_{1}^{k}}\left(\Pi_{2} \mid t_{2}, b_{2}\right)$ for every $b_{2} \in[6-\alpha, 6]$. Note that $C_{T}^{-1}\left(b_{2}\right) \in[0,1]$ is the unique type $t_{1}$ for bidder 1 such that $C_{T}\left(t_{1}\right)=b_{2}$. By 
the definition of $C_{T}$, we have $C_{T}^{-1}\left(b_{2}\right)=\sqrt{\frac{6-b_{2}}{\alpha}}$. Therefore, by (3.5)

$$
\begin{aligned}
\mathbf{E}_{\beta_{1}^{k}}\left(\Pi_{2} \mid t_{2}, b_{2}\right) & =\int_{0}^{1} \int_{5-\alpha}^{b_{2}}\left(5+t_{2}-\alpha t_{1}-b_{1}\right) \beta_{1}^{k}\left(t_{1}, d b_{1}\right) d t_{1} \\
& =\int_{0}^{C_{T}^{-1}\left(b_{2}\right)} \int_{5-\alpha}^{b_{2}}\left(5+t_{2}-\alpha t_{1}-b_{1}\right) \beta_{1}^{k}\left(t_{1}, d b_{1}\right) d t_{1} \\
& +\int_{C_{T}^{-1}\left(b_{2}\right)}^{1} \int_{5-\alpha}^{b_{2}}\left(5+t_{2}-\alpha t_{1}-b_{1}\right) \beta_{1}^{k}\left(t_{1}, d b_{1}\right) d t_{1} .
\end{aligned}
$$

From the definition of $\beta_{1}^{k}\left(t_{1}, \cdot\right)$ we obtain

$$
\begin{aligned}
I & =\left(1-2 \varepsilon_{k}-\varepsilon_{k}^{2}\right) \int_{0}^{C_{T}^{-1}\left(b_{2}\right)}\left(5+t_{2}-\alpha t_{1}-C_{M}\left(t_{1}\right)\right) d t_{1} \\
& +\varepsilon_{k} \int_{0}^{C_{T}^{-1}\left(b_{2}\right)}\left(5+t_{2}-\alpha t_{1}-C_{L}\left(t_{1}\right)\right) d t_{1} \\
& +\varepsilon_{k}^{2} \int_{0}^{C_{T}^{-1}\left(b_{2}\right)} \int_{5-\alpha}^{b_{2}}\left(5+t_{2}-\alpha t_{1}-b_{1}\right) \mu\left(d b_{1}\right) d t_{1} .
\end{aligned}
$$

Similarly, we have

$$
\begin{aligned}
\mathrm{II} & =\left(1-2 \varepsilon_{k}-\varepsilon_{k}^{2}\right) \int_{C_{T}^{-1}\left(b_{2}\right)}^{1}\left(5+t_{2}-\alpha t_{1}-C_{M}\left(t_{1}\right)\right) d t_{1} \\
& +\varepsilon_{k} \int_{C_{T}^{-1}\left(b_{2}\right)}^{1}\left(5+t_{2}-\alpha t_{1}-C_{L}\left(t_{1}\right)\right) d t_{1} \\
& +\varepsilon_{k}^{2} \int_{C_{T}^{-1}\left(b_{2}\right)}^{1} \int_{5-\alpha}^{b_{2}}\left(5+t_{2}-\alpha t_{1}-b_{1}\right) \mu\left(d b_{1}\right) d t_{1} \\
& +\varepsilon_{k} \int_{C_{T}^{-1}\left(b_{2}\right)}^{1}\left(5+t_{2}-\alpha t_{1}-C_{T}\left(t_{1}\right)\right) d t_{1} .
\end{aligned}
$$

Hence, by the definition of the curves $C_{M}, C_{T}$, and $C_{L}$ we obtain

$$
\begin{aligned}
\mathbf{E}_{\beta_{1}^{k}}\left(\Pi_{2} \mid t_{2}, b_{2}\right)=\mathrm{I}+\mathrm{II} & =\left(1-2 \varepsilon_{k}-\varepsilon_{k}^{2}\right) \int_{0}^{1}\left(5+t_{2}-\alpha t_{1}-5-(1-\alpha) t_{1}\right) d t_{1} \\
& +\varepsilon_{k} \int_{0}^{1}\left(5+t_{2}-\alpha t_{1}-5+\alpha-\alpha\left(1-t_{1}\right)^{2}\right) d t_{1} \\
& +\varepsilon_{k}^{2} \int_{0}^{1} \int_{5-\alpha}^{b_{2}}\left(5+t_{2}-\alpha t_{1}-b_{1}\right) \mu\left(d b_{1}\right) d t_{1} \\
& +\varepsilon_{k} \int_{C_{T}^{-1}\left(b_{2}\right)}^{1}\left(5+t_{2}-\alpha t_{1}-6+\alpha t_{1}^{2}\right) d t_{1} .
\end{aligned}
$$


Thus,

$$
\begin{aligned}
\mathbf{E}_{\beta_{1}^{k}}\left(\Pi_{2} \mid t_{2}, b_{2}\right) & =\left(1-2 \varepsilon_{k}-\varepsilon_{k}^{2}\right)\left(t_{2}-\frac{1}{2}\right)+\varepsilon_{k}\left(t_{2}+\frac{\alpha}{6}\right) \\
& +\frac{\varepsilon_{k}^{2}}{1+\alpha} \int_{5-\alpha}^{b_{2}}\left(5+t_{2}-\frac{\alpha}{2}-b_{1}\right) d b_{1} \\
& +\varepsilon_{k} \int_{C_{T}^{-1}\left(b_{2}\right)}^{1}\left(-1+t_{2}-\alpha t_{1}+\alpha t_{1}^{2}\right) d t_{1} .
\end{aligned}
$$

Note that for every $t_{2} \in[0,1]$

$$
\begin{aligned}
\mathbf{E}_{\beta_{1}^{k}}\left(\Pi_{2} \mid t_{2}, 6-\alpha\right)-\mathbf{E}_{\beta_{1}^{k}}\left(\Pi_{2} \mid t_{2}, 6\right) & =-\frac{\varepsilon_{k}^{2}}{1+\alpha} \int_{6-\alpha}^{6}\left(5+t_{2}-\frac{\alpha}{2}-b_{1}\right) d b_{1} \\
& -\varepsilon_{k} \int_{0}^{1}\left(-1+t_{2}-\alpha t_{1}+\alpha t_{1}^{2}\right) d t_{1} \\
& =\left(\frac{\alpha}{1+\alpha}\right) \varepsilon_{k}^{2}\left(1-t_{2}\right)+\varepsilon_{k}\left(1-t_{2}+\frac{\alpha}{6}\right),
\end{aligned}
$$

which is strictly positive and also that $\mathbf{E}_{\beta_{1}^{k}}\left(\Pi_{2} \mid t_{2}, b_{2}\right)$ is continuous on the compact set $\left(t_{2}, b_{2}\right) \in[0,1] \times[5-\alpha, 6]$. Therefore, there is a $y>0$ (without loss of generality we can assume that $y \in(0, \alpha-\xi)$, in order to make the analysis easier) such that for every $t_{2} \in[0,1]$ and $b_{2} \in(6-y, 6]$

$$
\mathbf{E}_{\beta_{1}^{k}}\left(\Pi_{2} \mid t_{2}, 6-\alpha\right)>\mathbf{E}_{\beta_{1}^{k}}\left(\Pi_{2} \mid t_{2}, b_{2}\right) .
$$

This means that for every $t_{2} \in[0,1]$, bidding $6-\alpha$ is strictly better than any bid in the interval $(6-y, 6]$. Hence, it suffices to prove Claim 1 over $b_{2} \in[6-\alpha, 6-y]$. We do so by examining the first derivative of $\mathbf{E}_{\beta_{1}^{k}}\left(\Pi_{2} \mid t_{2}, b_{2}\right)$ with respect to $b_{2}$, which equals to

$$
\frac{d}{d b_{2}} \mathbf{E}_{\beta_{1}^{k}}\left(\Pi_{2} \mid t_{2}, b_{2}\right)=\varepsilon_{k}^{2} \cdot q_{1}\left(t_{2}, b_{2}\right)+\varepsilon_{k} \cdot q_{2}\left(t_{2}, b_{2}\right),
$$

where

$$
\begin{aligned}
& q_{1}\left(t_{2}, b_{2}\right)=\frac{1}{1+\alpha} \cdot\left(5+t_{2}-\frac{\alpha}{2}-b_{2}\right) \\
& q_{2}\left(t_{2}, b_{2}\right)=\frac{1}{2 \alpha \cdot C_{T}^{-1}\left(b_{2}\right)} \cdot\left(-1+t_{2}-\alpha C_{T}^{-1}\left(b_{2}\right)+\alpha\left(C_{T}^{-1}\left(b_{2}\right)\right)^{2}\right) .
\end{aligned}
$$

Note that $q_{2}\left(t_{2}, b_{2}\right)$ is strictly negative for all $\left(t_{2}, b_{2}\right) \in[0,1] \times[6-\alpha, 6-y] \backslash$ $\{(1,6-\alpha)\}$, and $q_{2}(1,6-\alpha)=0$.

Because $q_{2}$ is strictly negative on the compact set $[0,1-\xi] \times[6-\alpha, 6-y]$, it has a strictly negative upper-bound on $[0,1-\xi] \times[6-\alpha, 6-y]$. Therefore, since 
$\varepsilon_{k}^{2}$ is relatively much smaller than $\varepsilon_{k}$ for sufficiently large $k$, then $\frac{d}{d b_{2}} \mathbf{E}_{\beta_{1}^{k}}\left(\Pi_{2} \mid\right.$ $\left.t_{2}, b_{2}\right)<0$ for all $\left(t_{2}, b_{2}\right) \in[0,1-\xi] \times[6-\alpha, 6-y]$. This proves that there exists an $N_{\xi}^{1} \in \mathbf{N}$ such that for every $k>N_{\xi}^{1}$ we have $\frac{d}{d b_{2}} \mathbf{E}_{\beta_{1}^{k}}\left(\Pi_{2} \mid t_{2}, b_{2}\right)<0$ for all $\left(t_{2}, b_{2}\right) \in[0,1-\xi] \times[6-\alpha, 6-y]$. Hence, part (1) of Claim 1 holds for all $k>N_{\xi}^{1}$.

Because $q_{2}$ is strictly negative on the compact set $[1-\xi, 1] \times[6-\alpha+\xi, 6-y]$, we can apply a similar argument and find an $L_{\xi}^{1} \in \mathbf{N}$ such that part (2) of Claim 1 holds for every $k>L_{\xi}^{1}$. Therefore, by choosing $M_{\xi}^{1}=\max \left\{N_{\xi}^{1}, L_{\xi}^{1}\right\}$, Claim 1 is valid for every $k>M_{\xi}^{1}$.

Proof of Claim 2: We compute $\mathbf{E}_{\beta_{1}^{k}}\left(\Pi_{2} \mid t_{2}, b_{2}\right)$ for every $b_{2} \in[5-\alpha, 5]$. Note that type $C_{L}^{-1}\left(b_{2}\right) \in[0,1]$ is the unique type $t_{1}$ for bidder 1 such that $C_{L}\left(t_{1}\right)=b_{2}$. By the definition of $C_{L}$, we have $C_{L}^{-1}\left(b_{2}\right)=1-\sqrt{\frac{b_{2}-5}{\alpha}+1}$. Hence, by (3.5)

$$
\begin{aligned}
\mathbf{E}_{\beta_{1}^{k}}\left(\Pi_{2} \mid t_{2}, b_{2}\right) & =\int_{0}^{1} \int_{5-\alpha}^{b_{2}}\left(5+t_{2}-\alpha t_{1}-b_{1}\right) \beta_{1}^{k}\left(t_{1}, d b_{1}\right) d t_{1} \\
& =\int_{0}^{C_{L}^{-1}\left(b_{2}\right)} \int_{5-\alpha}^{b_{2}}\left(5+t_{2}-\alpha t_{1}-b_{1}\right) \beta_{1}^{k}\left(t_{1}, d b_{1}\right) d t_{1} \\
& +\int_{C_{L}^{-1}\left(b_{2}\right)}^{1} \int_{5-\alpha}^{b_{2}}\left(5+t_{2}-\alpha t_{1}-b_{1}\right) \beta_{1}^{k}\left(t_{1}, d b_{1}\right) d t_{1} . \quad \text { IV }
\end{aligned}
$$

Therefore, we have

$$
\begin{aligned}
\mathrm{III} & =\varepsilon_{k}^{2} \int_{0}^{C_{L}^{-1}\left(b_{2}\right)} \int_{5-\alpha}^{b_{2}}\left(5+t_{2}-\alpha t_{1}-b_{1}\right) \mu\left(d b_{1}\right) d t_{1}, \\
\mathrm{IV} & =\varepsilon_{k} \int_{C_{L}^{-1}\left(b_{2}\right)}^{1}\left(5+t_{2}-\alpha t_{1}-C_{L}\left(t_{1}\right)\right) d t_{1} \\
& +\varepsilon_{k}^{2} \int_{C_{L}^{-1}\left(b_{2}\right)}^{1} \int_{5-\alpha}^{b_{2}}\left(5+t_{2}-\alpha t_{1}-b_{1}\right) \mu\left(d b_{1}\right) d t_{1} .
\end{aligned}
$$

Hence, by the definition of the curve $C_{L}$ we obtain

$$
\begin{aligned}
\mathbf{E}_{\beta_{1}^{k}}\left(\Pi_{2} \mid t_{2}, b_{2}\right)=\mathrm{III}+\mathrm{IV} & =\varepsilon_{k}^{2} \int_{0}^{1} \int_{5-\alpha}^{b_{2}}\left(5+t_{2}-\alpha t_{1}-b_{1}\right) \mu\left(d b_{1}\right) d t_{1} \\
& +\varepsilon_{k} \int_{C_{L}^{-1}\left(b_{2}\right)}^{1}\left(5+t_{2}-\alpha t_{1}-5+\alpha-\alpha\left(1-t_{1}\right)^{2}\right) d t_{1} \\
& =\frac{\varepsilon_{k}^{2}}{1+\alpha} \int_{5-\alpha}^{b_{2}}\left(5+t_{2}-\frac{\alpha}{2}-b_{1}\right) d b_{1} \\
& +\varepsilon_{k} \int_{C_{L}^{-1}\left(b_{2}\right)}^{1}\left(t_{2}+\alpha t_{1}-\alpha t_{1}^{2}\right) d t_{1} .
\end{aligned}
$$


Note that for every $t_{2} \in[0,1]$

$$
\begin{aligned}
\mathbf{E}_{\beta_{1}^{k}}\left(\Pi_{2} \mid t_{2}, 5\right)-\mathbf{E}_{\beta_{1}^{k}}\left(\Pi_{2} \mid t_{2}, 5-\alpha\right) & =\frac{\varepsilon_{k}^{2}}{1+\alpha} \int_{5-\alpha}^{5}\left(5+t_{2}-\frac{\alpha}{2}-b_{1}\right) d b_{1} \\
& +\varepsilon_{k} \int_{0}^{1}\left(t_{2}+\alpha t_{1}-\alpha t_{1}^{2}\right) d t_{1} \\
& =\left(\frac{\alpha}{1+\alpha}\right) \varepsilon_{k}^{2} t_{2}+\varepsilon_{k}\left(t_{2}+\frac{\alpha}{6}\right)>0
\end{aligned}
$$

and also that $\mathbf{E}_{\beta_{1}^{k}}\left(\Pi_{2} \mid t_{2}, b_{2}\right)$ is continuous on the compact set $\left(t_{2}, b_{2}\right) \in[0,1] \times$ $[5-\alpha, 5]$. Therefore, there is a $y>0$ (similar to the previous case assume that $y \in(0, \alpha-\xi))$ such that for every $t_{2} \in[0,1]$ and $b_{2} \in[5-\alpha, 5-\alpha+y)$

$$
\mathbf{E}_{\beta_{1}^{k}}\left(\Pi_{2} \mid t_{2}, 5\right)>\mathbf{E}_{\beta_{1}^{k}}\left(\Pi_{2} \mid t_{2}, b_{2}\right)
$$

This means that for every $t_{2} \in[0,1]$, bidding 5 is strictly better than any bid in the interval $[5-\alpha, 5-\alpha+y)$. Hence, it suffices to prove Claim 2 over the interval $[5-\alpha+y, 5]$. We do so by examining the first derivative of $\mathbf{E}_{\beta_{1}^{k}}\left(\Pi_{2} \mid t_{2}, b_{2}\right)$ with respect to $b_{2}$, which equals to

$$
\frac{d}{d b_{2}} \mathbf{E}_{\beta_{1}^{k}}\left(\Pi_{2} \mid t_{2}, b_{2}\right)=\varepsilon_{k}^{2} p_{1}\left(t_{2}, b_{2}\right)+\varepsilon_{k} p_{2}\left(t_{2}, b_{2}\right),
$$

where

$$
\begin{aligned}
& p_{1}\left(t_{2}, b_{2}\right)=\frac{1}{1+\alpha} \cdot\left(5+t_{2}-\frac{\alpha}{2}-b_{2}\right) \\
& p_{2}\left(t_{2}, b_{2}\right)=\frac{1}{2 \alpha \cdot\left(1-C_{L}^{-1}\left(b_{2}\right)\right)} \cdot\left(t_{2}+\alpha C_{L}^{-1}\left(b_{2}\right) 0-\alpha C_{L}^{-1}\left(b_{2}\right)^{2}\right) .
\end{aligned}
$$

Note that $p_{2}\left(t_{2}, b_{2}\right)$ is strictly positive for all $\left(t_{2}, b_{2}\right) \in[0,1] \times[5-\alpha+y, 5] \backslash$ $\{(0,5)\}$, and $p_{2}(0,5)=0$. With an argument similar to that of the previous case, we can find an $M_{\xi}^{2}$ such that for every $k>M_{\xi}^{2}$ Claim 2 is valid.

Proof of Claim 3: We compute $\mathbf{E}_{\beta_{1}^{k}}\left(\Pi_{2} \mid t_{2}, b_{2}\right)$ for every $b_{2} \in[5,6-\alpha]$. Note that $C_{M}^{-1}\left(b_{2}\right)=\frac{b_{2}-5}{1-\alpha}$ for every $b_{2} \in[5,6-\alpha]$. With a similar argument as before we have

$$
\begin{aligned}
\mathbf{E}_{\beta_{1}^{k}}\left(\Pi_{2} \mid t_{2}, b_{2}\right) & =\left(1-2 \varepsilon_{k}-\varepsilon_{k}^{2}\right) \int_{0}^{\frac{b_{2}-5}{1-\alpha}}\left(5+t_{2}-\alpha t_{1}-C_{M}\left(t_{1}\right)\right) d t_{1} \\
& +\varepsilon_{k} \int_{0}^{1}\left(5+t_{2}-\alpha t_{1}-C_{L}\left(t_{1}\right)\right) d t_{1} \\
& +\varepsilon_{k}^{2} \int_{0}^{1} \int_{5-\alpha}^{b_{2}}\left(5+t_{2}-\alpha t_{1}-b_{1}\right) \mu\left(d b_{1}\right) d t_{1} .
\end{aligned}
$$


Hence, by the definition of the curves $C_{M}$ and $C_{L}$ we obtain

$$
\begin{aligned}
\mathbf{E}_{\beta_{1}^{k}}\left(\Pi_{2} \mid t_{2}, b_{2}\right) & =\left(1-2 \varepsilon_{k}-\varepsilon_{k}^{2}\right) \int_{0}^{\frac{b_{2}-5}{1-\alpha}}\left(5+t_{2}-\alpha t_{1}-5-(1-\alpha) t_{1}\right) d t_{1} \\
& +\varepsilon_{k} \int_{0}^{1}\left(5+t_{2}-\alpha t_{1}-5+\alpha-\alpha\left(1-t_{1}\right)^{2}\right) d t_{1} \\
& +\varepsilon_{k}^{2} \int_{0}^{1} \int_{5-\alpha}^{b_{2}}\left(5+t_{2}-\alpha t_{1}-b_{1}\right) \mu\left(d b_{1}\right) d t_{1} .
\end{aligned}
$$

Thus,

$$
\begin{aligned}
\mathbf{E}_{\beta_{1}^{k}}\left(\Pi_{2} \mid t_{2}, b_{2}\right) & =\left(1-2 \varepsilon_{k}-\varepsilon_{k}^{2}\right) \int_{0}^{\frac{b_{2}-5}{1-\alpha}}\left(t_{2}-t_{1}\right) d t_{1} \\
& +\varepsilon_{k} \int_{0}^{1}\left(t_{2}+\alpha t_{1}-\alpha t_{1}^{2}\right) d t_{1} \\
& +\frac{\varepsilon_{k}^{2}}{1+\alpha} \int_{5-\alpha}^{b_{2}}\left(5+t_{2}-\frac{\alpha}{2}-b_{1}\right) d b_{1} \\
& =\left(1-2 \varepsilon_{k}-\varepsilon_{k}^{2}\right)\left(t_{2}\left(\frac{b_{2}-5}{1-\alpha}\right)-\frac{1}{2}\left(\frac{b_{2}-5}{1-\alpha}\right)^{2}\right) \\
& +\varepsilon_{k}\left(t_{2}+\frac{\alpha}{6}\right) \\
& +\frac{\varepsilon_{k}^{2}}{2(1+\alpha)}\left(b_{2}-5+\alpha\right)\left(5+2 t_{2}-b_{2}\right) .
\end{aligned}
$$

So, the first derivative of $\mathbf{E}_{\beta_{1}^{k}}\left(\Pi_{2} \mid t_{2}, b_{2}\right)$ with respect to $b_{2}$ is

$$
\begin{aligned}
& \frac{d}{d b_{2}} \mathbf{E}_{\beta_{1}^{k}}\left(\Pi_{2} \mid t_{2}, b_{2}\right) \\
= & \frac{1-2 \varepsilon_{k}-\varepsilon_{k}^{2}}{1-\alpha} \cdot\left(t_{2}-\frac{b_{2}-5}{1-\alpha}\right)+\frac{\varepsilon_{k}^{2}}{1+\alpha}\left(5+t_{2}-\frac{\alpha}{2}-b_{2}\right) .
\end{aligned}
$$

First, we prove that for every $t_{2} \in[\xi, 1-\xi]$ the maximum of $\mathbf{E}_{\beta_{1}^{k}}\left(\Pi_{2} \mid t_{2}, b_{2}\right)$ cannot be attained on the boundary of interval $[5,6-\alpha]$. Observe that

$$
\frac{d}{d b_{2}} \mathbf{E}_{\beta_{1}^{k}}\left(\Pi_{2} \mid t_{2}, 5\right)=\frac{1-2 \varepsilon_{k}-\varepsilon_{k}^{2}}{1-\alpha} \cdot t_{2}+\frac{\varepsilon_{k}^{2}}{1+\alpha}\left(t_{2}-\frac{\alpha}{2}\right),
$$

and

$$
\frac{d}{d b_{2}} \mathbf{E}_{\beta_{1}^{k}}\left(\Pi_{2} \mid t_{2}, 6-\alpha\right)=\frac{1-2 \varepsilon_{k}-\varepsilon_{k}^{2}}{1-\alpha} \cdot\left(t_{2}-1\right)+\frac{\varepsilon_{k}^{2}}{1+\alpha}\left(t_{2}-1+\frac{\alpha}{2}\right) .
$$

Hence, there is an $N_{\xi}^{3} \in \mathbf{N}$ such that if $k>N_{\xi}^{3}$ then for every $t_{2} \in[\xi, 1-\xi]$ we have

$$
\frac{d}{d b_{2}} \mathbf{E}_{\beta_{1}^{k}}\left(\Pi_{2} \mid t_{2}, 5\right)>0
$$


and

$$
\frac{d}{d b_{2}} \mathbf{E}_{\beta_{1}^{k}}\left(\Pi_{2} \mid t_{2}, 6-\alpha\right)<0
$$

Therefore, by the continuity of $\mathbf{E}_{\beta_{1}^{k}}\left(\Pi_{2} \mid t_{2}, b_{2}\right)$, we can conclude for every $t_{2} \in$ $[\xi, 1-\xi]$ that if $b_{2}$ maximizes $\mathbf{E}_{\beta_{1}^{k}}\left(\Pi_{2} \mid t_{2}, b_{2}\right)$, then

$$
\frac{d}{d b_{2}} \mathbf{E}_{\beta_{1}^{k}}\left(\Pi_{2} \mid t_{2}, b_{2}\right)=0
$$

One can check with the help of (3.11) that the unique solution of (3.12), for every $t_{2} \in[0,1]$, is

$$
b_{2}\left(t_{2}\right)=\frac{\left(1-2 \varepsilon_{k}-\varepsilon_{k}^{2}\right)(1+\alpha)}{v\left(\varepsilon_{k}\right)} \cdot\left(5+(1-\alpha) t_{2}\right)+\frac{\varepsilon_{k}^{2}(1-\alpha)^{2}}{v\left(\varepsilon_{k}\right)}\left(5+t_{2}-\frac{\alpha}{2}\right),
$$

where

$$
v\left(\varepsilon_{k}\right)=\left(1-2 \varepsilon_{k}-\varepsilon_{k}^{2}\right)(1+\alpha)+\varepsilon_{k}^{2}(1-\alpha)^{2} .
$$

One can verify that

$$
\left|b_{2}\left(t_{2}\right)-C_{M}\left(t_{2}\right)\right|=\frac{\alpha \varepsilon_{k}^{2}(1-\alpha)^{2}}{v\left(\varepsilon_{k}\right)}\left|t_{2}-\frac{1}{2}\right| \leq \frac{\alpha \varepsilon_{k}^{2}(1-\alpha)^{2}}{2 v\left(\varepsilon_{k}\right)} .
$$

Therefore, there is an $L_{\xi}^{3} \in \mathbf{N}$ such that for every $k>L_{\xi}^{3}$ and every $t_{2} \in[0,1]$ we have

$$
\left|b_{2}\left(t_{2}\right)-C_{M}\left(t_{2}\right)\right| \leq \xi(1-\alpha) \text {. }
$$

By choosing $M_{\xi}^{3}=\max \left\{N_{\xi}^{3}, L_{\xi}^{3}\right\}$, for every $k>M_{\xi}^{3}$, part (1) of Claim 3 is valid.

Since at $t_{2}=0$, for every $b_{2} \in[5,6-\alpha]$ we have

$$
\frac{d}{d b_{2}} \mathbf{E}_{\beta_{1}^{k}}\left(\Pi_{2} \mid 0, b_{2}\right)=\frac{1-2 \varepsilon_{k}-\varepsilon_{k}^{2}}{1-\alpha} \cdot\left(-\frac{b_{2}-5}{1-\alpha}\right)+\frac{\varepsilon_{k}^{2}}{1+\alpha}\left(5-\frac{\alpha}{2}-b_{2}\right)<0
$$

the maximum at $t_{2}=0$ is obtained at $b_{2}=5$. Moreover, by part (1) of Claim 3 we have that if $k>\max \left\{N_{\xi}^{3}, L_{\xi}^{3}\right\}$, then for $t_{2}=\xi$ the maximum is obtained within $b_{2} \in[5,5+2(1-\alpha) \xi]$. One can verify that in (3.11), the first derivative of unique solution $b_{2}\left(t_{2}\right)$ is strictly positive, then we can conclude that for every $t_{2} \in[0, \xi)$ the maximum is obtained within $b_{2} \in[5,5+2(1-\alpha) \xi]$, for large $k$.

With a similar argument, since at $t_{2}=1$ for every $b_{2} \in[5,6-\alpha]$ we have

$$
\frac{d}{d b_{2}} \mathbf{E}_{\beta_{1}^{k}}\left(\Pi_{2} \mid 1, b_{2}\right)=\frac{1-2 \varepsilon_{k}-\varepsilon_{k}^{2}}{1-\alpha} \cdot\left(1-\frac{b_{2}-5}{1-\alpha}\right)+\frac{\varepsilon_{k}^{2}}{1+\alpha}\left(6-\frac{\alpha}{2}-b_{2}\right)>0,
$$


the maximum at $t_{2}=1$ is obtained in $b_{2}=6-\alpha$. Moreover, if $k>\max \left\{N_{\xi}^{3}, L_{\xi}^{3}\right\}$, then for $t_{2}=1-\xi$ the maximum is obtained within $b_{2} \in[6-\alpha-2(1-\alpha) \xi, 6-\alpha]$. Similarly, since $b_{2}{ }^{\prime}\left(t_{2}\right)$ is strictly positive, we conclude that for every $t_{2} \in(1-\xi, 1]$ the maximum is obtained within $b_{2} \in[6-\alpha-2(1-\alpha) \xi, 6-\alpha]$ for large $k$. This complete the proof of Claim 3 , for every $k>M_{\xi}^{3}$.

\section{Proofs of Propositions 3.4.6 and 3.4.7}

It is easy to check that strategy profiles $\sigma$ and $\eta$ are BNEs.

We now prove that the BNE $\sigma=\left(\sigma_{1}, \sigma_{2}\right)$, in which $\sigma_{1}\left(t_{1}, \cdot\right)=\delta_{6}(\cdot)$ and $\sigma_{2}\left(t_{2}, \cdot\right)=\delta_{5-\alpha}(\cdot)$, is not pointwise-perfect, consequently is not uniform-perfect. Let $\left(\sigma_{1}^{k}\right)_{k=1}^{\infty}$ be a sequence of completely mixed strategies for bidder 1 such that $\rho^{w}\left(\sigma_{1}^{k}, \sigma_{1}\right) \longrightarrow 0$ as $k \rightarrow \infty$. We show that bidder's 2 best response against $\sigma_{1}^{k}$ does not converge to $\sigma_{2}$ when $k \rightarrow \infty$.

Suppose that bidder 1 plays $\sigma_{1}^{k}$, for some $k \in \mathbf{N}$. We prove that for $t_{2}=1$, the best response of bidder 2 is far from $\sigma_{2}\left(t_{2}, \cdot\right)=\delta_{5-\alpha}(\cdot)$. Note that bidder 2 gets always zero by choosing $\sigma_{2}$, because he never wins. So, it is enough to show that his expected profit is strictly positive by bidding $6-\alpha$, when $t_{2}=1$. We compute bidder 2's expected profit given type $t_{2}=1$ and bid $b_{2}=6-\alpha$.

$$
\mathbf{E}_{\sigma_{1}^{k}}\left(\Pi_{2} \mid 1,6-\alpha\right)=\int_{0}^{1} \int_{5-\alpha}^{6-\alpha}\left(5+1-\alpha t_{1}-b_{1}\right) \sigma_{1}^{k}\left(t_{1}, d b_{1}\right) d t_{1} .
$$

Since $\sigma_{1}^{k}$ is a completely mixed strategy we have $\sigma_{1}^{k}\left(t_{1},(5-\alpha, 6-\alpha)\right)>0$, therefore by using the fact that $b_{1} \in(5-\alpha, 6-\alpha)$ we have

$$
\begin{aligned}
\mathbf{E}_{\sigma_{1}^{k}}\left(\Pi_{2} \mid 1,6-\alpha\right) & >\int_{0}^{1} \int_{5-\alpha}^{6-\alpha}\left(5+1-\alpha t_{1}-6+\alpha\right) \sigma_{1}^{k}\left(t_{1}, d b_{1}\right) d t_{1} \\
& =\alpha \int_{0}^{1} \int_{5-\alpha}^{6-\alpha}\left(1-t_{1}\right) \sigma_{1}^{k}\left(t_{1}, d b_{1}\right) d t_{1} \geq 0 .
\end{aligned}
$$

This implies that $\mathbf{E}_{\sigma_{1}^{k}}\left(\Pi_{2} \mid 1,6-\alpha\right)>0$, hence $\sigma_{2}$ is not pointwise-perfect.

With a similar argument, one can prove that the discontinuous BNE $\eta$ is not pointwise-perfect, and hence not uniform-perfect either.

\subsection{Appendix II: A measurability result for the weak distance}

A metric space $(X, d)$ is called $\sigma$-compact, if $X$ is a countable union of compact subsets of $X$.

A strategy $\beta_{i}$ for some player $i$ is called simple if there exists a finite set $B \subseteq A_{i}$ such that $\beta_{i}\left(t_{i}, B\right)=1$ for every $t_{i} \in T_{i}$. 
Lemma 3.6.1 Assume that the action space $A_{i}$ is $\sigma$-compact for a player $i \in N$. Then, for every strategy $\beta_{i}$ for player $i$, there exists a sequence $\left(\tau_{i}^{k}\right)_{k=1}^{\infty}$ of simple strategies such that

$$
\lim _{k \rightarrow \infty} \rho^{w}\left(\tau_{i}^{k}\left(t_{i}, \cdot\right), \beta_{i}\left(t_{i}, \cdot\right)\right)=0
$$

for every type $t_{i} \in T_{i}$.

Proof. First we define the sequence $\left(\tau_{i}^{k}\right)_{k=1}^{\infty}$ and then we prove that this sequence satisfies equality (3.13).

Part 1: The definition of the sequence $\left(\tau_{i}^{k}\right)_{k=1}^{\infty}$ : Since $A_{i}$ is $\sigma$-compact, $A_{i}=$ $\cup_{j=1}^{\infty} K_{j}$ for some compact sets $K_{j} \subseteq A_{i}$. Let $A_{i}^{k}=\cup_{j=1}^{k} K_{j}$ for every $k \in \mathbb{N}$. Note that $A_{i}^{k}$ is compact for every $k \in \mathbb{N}$, the sequence $\left(A_{i}^{k}\right)_{k=1}^{\infty}$ is increasing and $A_{i}=\cup_{k=1}^{\infty} A_{i}^{k}$.

Take a $k \in \mathbb{N}$. For every $a \in A_{i}^{k}$, let $U^{k}(a)=\left\{a^{\prime} \in A_{i} \mid d_{A_{i}}\left(a, a^{\prime}\right)<\frac{1}{k}\right\}$. Since $A_{i}^{k}$ is compact, there exist actions $a_{1}^{k}, \ldots, a_{J^{k}}^{k} \in A_{i}^{k}$ such that

$$
A_{i}^{k} \subseteq \bigcup_{j=1}^{J^{k}} U^{k}\left(a_{j}^{k}\right)
$$

Define $B_{1}^{k}=U^{k}\left(a_{1}^{k}\right) \cap A_{i}^{k}$, and $B_{j}^{k}=\left[U^{k}\left(a_{j}^{k}\right) \cap A_{i}^{k}\right] \backslash \cup_{\ell=1}^{j-1} U^{k}\left(a_{\ell}^{k}\right)$ for every $j=2, \ldots, J^{k}$. Without loss of generality we can assume, for every $j$, that $B_{j}^{k}$ is non-empty; otherwise we could leave out $a_{j}^{k}$ from the list $a_{1}^{k}, \ldots, a_{J^{k}}^{k}$. Thus, $\left\{B_{1}^{k}, \ldots, B_{J^{k}}^{k}\right\}$ forms a partition of $A_{i}^{k}$. Moreover, by construction, $B_{j}^{k} \in \mathcal{A}_{i}$ for every $j=1, \ldots, J^{k}$. Take an arbitrary $b_{j}^{k} \in B_{j}^{k}$ for every $j=1, \ldots, J^{k}$.

Now define the simple strategy $\tau_{i}^{k}$ for player $i$ by

$$
\tau_{i}^{k}\left(t_{i}, \cdot\right)=\beta_{i}\left(t_{i}, B_{1}^{k}\right) \cdot \delta_{b_{1}^{k}}(\cdot)+\ldots+\beta_{i}\left(t_{i}, B_{J^{k}}^{k}\right) \cdot \delta_{b_{J}^{k}}(\cdot)+\left(1-\beta_{i}\left(t_{i}, A_{i}^{k}\right)\right) \cdot \delta_{b_{1}^{k}}(\cdot)
$$

for every $t_{i} \in T_{i}$, where as usual, $\delta$ stands for the Dirac measure. Note that $\tau_{i}^{k}$ satisfies condition (2) of the definition of behavioral strategies. The last term in the definition of $\tau_{i}^{k}\left(t_{i}, \cdot\right)$ will not play an important role, but it is needed so that $\tau_{i}^{k}\left(t_{i}, \cdot\right)$ is a probability measure.

Part 2: The proof that the sequence $\left(\tau_{i}^{k}\right)_{k=1}^{\infty}$ satisfies equality (3.13): Fix a type $t_{i} \in T_{i}$. Consider a bounded Lipschitz function $f: A_{i} \rightarrow \mathbb{R}$. Then, there exist $C_{1}, C_{2} \geq 0$ such that $|f(a)| \leq C_{1}$ and $\left|f(a)-f\left(a^{\prime}\right)\right| \leq C_{2} \cdot d_{A_{i}}\left(a, a^{\prime}\right)$ for every $a, a^{\prime} \in A_{i}$. Let

$$
D^{k}=\left|\int_{A_{i}} f(a) \tau_{i}^{k}\left(t_{i}, d a\right)-\int_{A_{i}} f(a) \beta_{i}\left(t_{i}, d a\right)\right| .
$$


Since

$$
\int_{A_{i}} f(a) \tau_{i}^{k}\left(t_{i}, d a\right)=\sum_{j=1}^{J^{k}} f\left(b_{j}^{k}\right) \cdot \beta_{i}\left(t_{i}, B_{j}^{k}\right)+f\left(b_{1}^{k}\right) \cdot\left(1-\beta_{i}\left(t_{i}, A_{i}^{k}\right)\right)
$$

and

$$
\int_{A_{i}} f(a) \beta_{i}\left(t_{i}, d a\right)=\sum_{j=1}^{J^{k}} \int_{B_{j}^{k}} f(a) \beta_{i}\left(t_{i}, d a\right)+\int_{A_{i} \backslash A_{i}^{k}} f(a) \beta_{i}\left(t_{i}, d a\right),
$$

we have

$$
\begin{aligned}
D^{k} & \leq \sum_{j=1}^{J^{k}}\left|f\left(b_{j}^{k}\right) \cdot \beta_{i}\left(t_{i}, B_{j}^{k}\right)-\int_{B_{j}^{k}} f(a) \beta_{i}\left(t_{i}, d a\right)\right|+2 C_{1} \cdot\left(1-\beta_{i}\left(t_{i}, A_{i}^{k}\right)\right) \\
& \leq \sum_{j=1}^{J^{k}}\left|\int_{B_{j}^{k}}\left[f\left(b_{j}^{k}\right)-f(a)\right] \beta_{i}\left(t_{i}, d a\right)\right|+2 C_{1} \cdot\left(1-\beta_{i}\left(t_{i}, A_{i}^{k}\right)\right) \\
& \leq \sum_{j=1}^{J^{k}} \sup _{a \in B_{j}^{k}}\left|f\left(b_{j}^{k}\right)-f(a)\right| \cdot \beta_{i}\left(t_{i}, B_{j}^{k}\right)+2 C_{1} \cdot\left(1-\beta_{i}\left(t_{i}, A_{i}^{k}\right)\right) .
\end{aligned}
$$

Since the diameter of $B_{j}^{k}$, for each $j=1, \ldots, J^{k}$, is at most $\frac{2}{k}$, it follows that

$$
\begin{aligned}
D^{k} & \leq \sum_{j=1}^{J^{k}} \frac{2}{k} \cdot C_{2} \cdot \beta_{i}\left(t_{i}, B_{j}^{k}\right)+2 C_{1} \cdot\left(1-\beta_{i}\left(t_{i}, A_{i}^{k}\right)\right) \\
& =\frac{2}{k} \cdot C_{2} \cdot \beta_{i}\left(t_{i}, A_{i}^{k}\right)+2 C_{1} \cdot\left(1-\beta_{i}\left(t_{i}, A_{i}^{k}\right)\right)
\end{aligned}
$$

By the continuity of the measure, $\beta_{i}\left(t_{i}, A_{i}^{k}\right)$ converges to $\beta_{i}\left(t_{i}, A_{i}\right)=1$ as $k \rightarrow \infty$, and hence $D^{k}$ converges to zero as $k \rightarrow \infty$. As $f$ was an arbitrary bounded Lipschitz function from $A_{i}$ to $\mathbf{R}$, the proof of (3.13) is complete.

Lemma 3.6.2 Assume that the action space $A_{i}$ is $\sigma$-compact for a player $i \in N$, and let $\beta_{i}^{1}$ and $\beta_{i}^{2}$ be two strategies for player $i$. Then, the function $f: T_{i} \rightarrow \mathbb{R}$ defined by $f\left(t_{i}\right)=\rho^{w}\left(\beta_{i}^{1}\left(t_{i}, \cdot\right), \beta_{i}^{2}\left(t_{i}, \cdot\right)\right)$ is measurable.

Proof. It suffices to prove that the set $\left\{t_{i} \in T_{i} \mid f\left(t_{i}\right)>r\right\}$ is measurable for every $r \in \mathbb{R}$. So, fix an arbitrary $r \in \mathbf{R}$.

Part 1: when $\beta_{i}^{1}$ and $\beta_{i}^{2}$ are simple strategies.

First assume that $\beta_{i}^{1}$ and $\beta_{i}^{2}$ are simple strategies. Then, there are finite sets $B^{1}, B^{2} \subseteq$ 
$A_{i}$ such that $\beta_{i}^{1}\left(t_{i}, B^{1}\right)=1$ and $\beta_{i}^{2}\left(t_{i}, B^{2}\right)=1$ for every $t_{i} \in T_{i}$. Let $B=B^{1} \cup B^{2}$. We have

$$
\begin{array}{r}
f\left(t_{i}\right)=\inf \left\{\varepsilon>0 \mid \beta_{i}^{1}\left(t_{i}, C\right) \leq \beta_{i}^{2}\left(t_{i}, C^{\varepsilon}\right)+\varepsilon\right. \text { and } \\
\left.\beta_{i}^{2}\left(t_{i}, C\right) \leq \beta_{i}^{1}\left(t_{i}, C^{\varepsilon}\right)+\varepsilon \forall C \in \mathcal{A}_{i}\right\} .
\end{array}
$$

Notice that if $\beta_{i}^{1}\left(t_{i}, C\right) \leq \beta_{i}^{2}\left(t_{i}, C^{\varepsilon}\right)+\varepsilon$ for some $C \subseteq B$, then for any $\widetilde{C} \in \mathcal{A}_{i}$ satisfying $\widetilde{C} \cap B=C$, we obtain

$$
\beta_{i}^{1}\left(t_{i}, \widetilde{C}\right)=\beta_{i}^{1}\left(t_{i}, C\right) \leq \beta_{i}^{2}\left(t_{i}, C^{\varepsilon}\right)+\varepsilon \leq \beta_{i}^{2}\left(t_{i}, \widetilde{C}^{\varepsilon}\right)+\varepsilon
$$

Similarly, if $\beta_{i}^{2}\left(t_{i}, C\right) \leq \beta_{i}^{1}\left(t_{i}, C^{\varepsilon}\right)+\varepsilon$ then

$$
\beta_{i}^{2}\left(t_{i}, \widetilde{C}\right) \leq \beta_{i}^{1}\left(t_{i}, \widetilde{C}^{\varepsilon}\right)+\varepsilon .
$$

Hence,

$$
\begin{array}{r}
f\left(t_{i}\right)=\inf \left\{\varepsilon>0 \mid \beta_{i}^{1}\left(t_{i}, C\right) \leq \beta_{i}^{2}\left(t_{i}, C^{\varepsilon}\right)+\varepsilon\right. \text { and } \\
\left.\beta_{i}^{2}\left(t_{i}, C\right) \leq \beta_{i}^{1}\left(t_{i}, C^{\varepsilon}\right)+\varepsilon \forall C \subseteq B\right\} .
\end{array}
$$

Notice that $f\left(t_{i}\right)>r$ holds if and only if there exists an $m \in \mathbf{N}$ and a set $C \subseteq B$ such that for $\varepsilon=r+\frac{1}{m}$ we have either $\beta_{i}^{1}\left(t_{i}, C\right)>\beta_{i}^{2}\left(t_{i}, C^{\varepsilon}\right)+\varepsilon$ or $\beta_{i}^{2}\left(t_{i}, C\right)>\beta_{i}^{1}\left(t_{i}, C^{\varepsilon}\right)+\varepsilon$. Indeed, the "only if"-part is immediate, whereas the "if"-part follows from the fact that $\beta_{i}^{2}\left(t_{i}, C^{\varepsilon}\right)+\varepsilon$ and $\beta_{i}^{1}\left(t_{i}, C^{\varepsilon}\right)+\varepsilon$ are increasing in $\varepsilon$.

For every $m \in \mathbf{N}$ and $C \subseteq B$, define

$$
\begin{aligned}
& U_{m, C}^{1}=\left\{t_{i} \in T_{i} \mid \beta_{i}^{1}\left(t_{i}, C\right)>\beta_{i}^{2}\left(t_{i}, C^{r+\frac{1}{m}}\right)+r+\frac{1}{m}\right\} \\
& U_{m, C}^{2}=\left\{t_{i} \in T_{i} \mid \beta_{i}^{2}\left(t_{i}, C\right)>\beta_{i}^{1}\left(t_{i}, C^{r+\frac{1}{m}}\right)+r+\frac{1}{m}\right\} .
\end{aligned}
$$

Since by condition (2) of the definition of behavioral strategies, the section functions $\beta_{i}^{1}(\cdot, C): T_{i} \rightarrow \mathbb{R}$ and $\beta_{i}^{2}\left(\cdot, C^{r+\frac{1}{m}}\right): T_{i} \rightarrow \mathbb{R}$ are measurable, the sets $U_{m, C}^{1}$ are measurable. For a similar reason, the sets $U_{m, C}^{2}$ are measurable too. Now we have

$$
\left\{t_{i} \in T_{i} \mid f\left(t_{i}\right)>r\right\}=\bigcup_{m=1}^{\infty} \bigcup_{C \subseteq B}\left[U_{m, C}^{1} \bigcup U_{m, C}^{2}\right] .
$$

Because the right hand side is a countable union of measurable sets, the set $\left\{t_{i} \in\right.$ $\left.T_{i} \mid f\left(t_{i}\right)>r\right\}$ is measurable as well. So, the claim of the lemma holds for simple strategies. 
Part 2: when $\beta_{i}^{1}$ and $\beta_{i}^{2}$ are arbitrary strategies.

Now we prove the lemma for arbitrary strategies $\beta_{i}^{1}$ and $\beta_{i}^{2}$. By Lemma 3.6.1, there are two sequences of simple strategies $\left(\tau_{i}^{k, 1}\right)_{k=1}^{\infty}$ and $\left(\tau_{i}^{k, 2}\right)_{k=1}^{\infty}$ such that

$$
\lim _{k \rightarrow \infty} \rho^{w}\left(\tau_{i}^{k, 1}\left(t_{i}, \cdot\right), \beta_{i}^{1}\left(t_{i}, \cdot\right)\right)=0, \quad \text { and } \quad \lim _{k \rightarrow \infty} \rho^{w}\left(\tau_{i}^{k, 2}\left(t_{i}, \cdot\right), \beta_{i}^{2}\left(t_{i}, \cdot\right)\right)=0
$$

for every type $t_{i} \in T_{i}$. By the definition of $f$, we have

$$
\left\{t_{i} \in T_{i} \mid f\left(t_{i}\right)>r\right\}=\left\{t_{i} \in T_{i} \mid \lim _{k \rightarrow \infty} \rho^{w}\left(\tau_{i}^{k, 1}\left(t_{i}, \cdot\right), \tau_{i}^{k, 2}\left(t_{i}, \cdot\right)\right)>r\right\} .
$$

Notice that, for some $t_{i} \in T_{i}$, the inequality

$$
\lim _{k \rightarrow \infty} \rho^{w}\left(\tau_{i}^{k, 1}\left(t_{i}, \cdot\right), \tau_{i}^{k, 2}\left(t_{i}, \cdot\right)\right)>r
$$

holds if and only if there exists an $m \in \mathbb{N}$ such that $\rho^{w}\left(\tau_{i}^{k, 1}\left(t_{i}, \cdot\right), \tau_{i}^{k, 2}\left(t_{i}, \cdot\right)\right)>$ $r+\frac{1}{m}$ holds for sufficiently large $k$. Therefore,

$$
\left\{t_{i} \in T_{i} \mid f\left(t_{i}\right)>r\right\}=\bigcup_{m=1}^{\infty} \bigcup_{\ell=1}^{\infty} \bigcap_{k=\ell}^{\infty}\left\{t_{i} \in T_{i} \mid \rho^{w}\left(\tau_{i}^{k, 1}\left(t_{i}, \cdot\right), \tau_{i}^{k, 2}\left(t_{i}, \cdot\right)\right)>r+\frac{1}{m}\right\} .
$$

For each $k \in \mathbb{N}$, because the strategies $\tau_{i}^{k, 1}$ and $\tau_{i}^{k, 2}$ are simple, part 1 of the proof implies that set

$$
\left\{t_{i} \in T_{i} \mid \rho^{w}\left(\tau_{i}^{k, 1}\left(t_{i}, \cdot\right), \tau_{i}^{k, 2}\left(t_{i}, \cdot\right)\right)>r+\frac{1}{m}\right\}
$$

is measurable. Therefore, the set $\left\{t_{i} \in T_{i} \mid f\left(t_{i}\right)>r\right\}$ is measurable as well. 


\section{Chapter 4}

\section{Distributional perfect equilibrium in Bayesian games}

\subsection{Introduction}

In private value second-price auctions each bidder has a dominant strategy in which he bids his own valuation of the object. However, in second-price auctions with interdependent values bidders might not have any dominant strategy while there may exist many equilibria. Therefore, a selection tool is needed in such Bayesian games to rule out the less intuitive equilibria. In this chapter we develop the concept of trembling hand perfect equilibrium for Bayesian games with infinite type and action spaces as a refinement of Bayesian Nash equilibrium.

We consider a Bayesian game with $n$ players, where each player's type space is a separable metric space. After receiving the types, players simultaneously choose an action from a compact metric action space, then each player gets a payoff that may depend on all the types and all the actions chosen by the players. Following Milgrom and Weber [33], we assume that the prior probability measure on the product of the type spaces is absolutely continuous with respect to the product measure of its marginal probabilities, also we assume that the payoffs are equicontinuous. Milgrom and Weber in [33] prove the existence of an equilibrium in distributional strategies for such Bayesian games. ${ }^{1}$ As it is not clear how players would play the game when using their distributional strategies, we define a similar equilibrium concept in behavioral strategies. A behavioral strategy profile is called "distributional equilibrium" if its induced distributional strategy profile is an equilibrium.

\footnotetext{
${ }^{1}$ There are many other studies on the existence of equilibrium in Bayesian games, for example: Athey [3], Mcadams [25], Reny [40], Jackson et. al. [16],[17], Mertens [31], Meirowitz [26], Mallozzi et. al. [24], Kim and Yannelis [20], Zandt and Vives [45], Balder [5].
} 
To extend the concept of trembling hand perfect equilibrium to Bayesian games with infinite action and type spaces, we define "distributional perfect equilibrium" as a behavioral strategy profile such that for its induced distributional strategy profile the conditions of trembling hand perfect equilibrium are satisfied. We prove that in the Bayesian game described above distributional perfection is a refinement of distributional equilibrium. Furthermore, we show the existence of distributional perfect equilibrium and that the set of distributional perfect equilibria is sequentially closed in the Tychonoff topology when taking the strong topology on the set of probability measures on action spaces.

Note that in finite games perfect equilibrium assigns no mass to weakly dominated strategies. However, this property is not compatible for infinite games, as Example 2.1 by Simon and Stinchcombe in [42] presents a game with infinite action spaces in which the unique equilibrium of the game is in weakly dominated strategies. Instead, the weaker property of limit undominatedness ${ }^{2}$ is used for strategies in infinite games which is somewhat stronger than limit admissibility introduced in Simon and Stinchcombe [42]. We show that a distributional perfect equilibrium is in limit undominated strategies. We also discuss perfection in finite Bayesian games, to see that distributional perfection is equivalent to perfect equilibrium in finite Bayesian games defined in chapter 3 if every player receives each type from his type space with strictly positive probability.

As we mentioned before, one of the applications of distributional perfection is in auctions with interdependent values, because such auctions may have many equilibria, even in undominated strategies. We study symmetric second-price auctions with interdependent values for two bidders where the valuation function of each bidder is strictly increasing in his own type. We use distributional perfection to get a smaller set of equilibria and rule out the strange and the less intuitive ones. To explain this idea consider the following example.

Example 4.1.1 Consider a 2-bidder second-price auction with interdependent values $v_{1}=t_{1}+\frac{1}{2} t_{2}$ and $v_{2}=\frac{1}{2} t_{1}+t_{2}$, where the types $t_{1}, t_{2}$ for bidder 1 and 2 respectively, are drawn independently from $[0,1]$ according to the uniform distribution. Then, each bidder simultaneously submits a bid from the set $\left[0, \frac{3}{2}\right]$. The strategy profile $\left(b_{1}, b_{2}\right)$ in which $b_{i}=\frac{3}{2} t_{i}$, for every bidder $i=1,2$, is a Bayesian Nash equilibrium. However, there are many other equilibria in this auction. As an example consider the following class of equilibria. For every $s_{1}, s_{2} \in[0,1]$ where $s_{1}<s_{2}$ :

\footnotetext{
${ }^{2}$ This property was introduced in chapter 2 .
} 


$$
\begin{aligned}
& \hat{b}_{1}\left(t_{1}\right)= \begin{cases}s_{2}+\frac{1}{2} t_{1} & \text { if } t_{1} \in\left[s_{1}, s_{2}\right] \\
\frac{3}{2} t_{1} & \text { otherwise }\end{cases} \\
& \hat{b}_{2}\left(t_{2}\right)= \begin{cases}s_{1}+\frac{1}{2} t_{2} & \text { if } t_{2} \in\left[s_{1}, s_{2}\right] \\
\frac{3}{2} t_{2} & \text { otherwise. }\end{cases}
\end{aligned}
$$

This class of equilibria is depicted in Figure 4.1. In this strategy profile, in the case both types are drawn from the set $\left[s_{1}, s_{2}\right]$, bidder 1 wins the auction and pays the amount $\hat{b}_{2}\left(t_{2}\right)=s_{1}+\frac{1}{2} t_{2}$, which is his minimum ex post valuation in this case. Note that in this case bidder 1 overbids, while bidder 2 underbids. This class of equilibria leads to an inefficient outcome, hence we would like to be able to rule them out by the help of some kind of refinement.

In Proposition 4.5.1, we will show that the strategy profile $\left(b_{1}, b_{2}\right)$ is distributional perfect. Moreover, in Proposition 4.5.3 we will see that a large subclass of strategy profiles $\left(\hat{b}_{1}, \hat{b}_{2}\right)$ are not distributional perfect. The reason for this observation is that in the latter equilibria, bidder 1 might bid even higher than his maximum valuation and bidder 2 might bid even lower than his minimum valuation. Now, if each bidder takes making mistakes by his opponent serious, then he knows any bid from the set $\left[0, \frac{3}{2}\right]$ is possible. This prevents bidder 1 from overbidding, since by overbidding he may get a negative payoff at the end of the day. Similarly, by taking mistakes into account by bidder 2 , he would not underbid anymore, as he can increase his chance of winning and getting a strictly positive payoff.
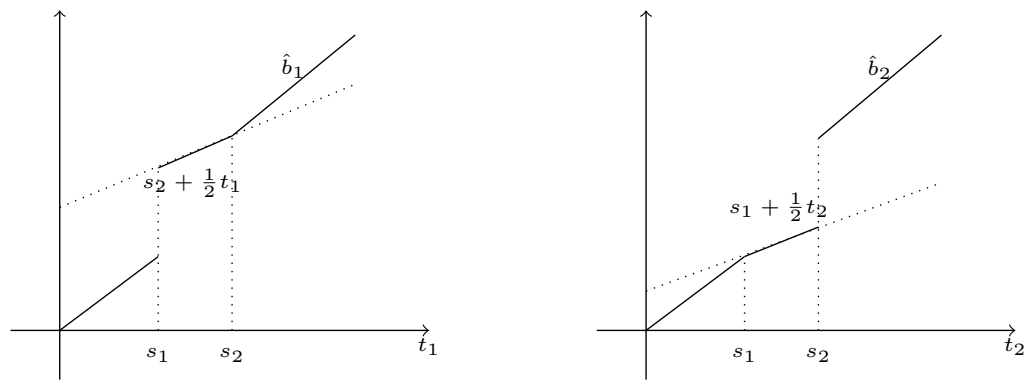

Figure 4.1

By taking a different approach in chapter 3, we define perfect equilibrium for Bayesian games only based on behavioral strategies and without making use of distributional strategies so called behavioral perfection. Behavioral perfection is a more 
straightforward way of defining perfect equilibrium in Bayesian games than distributional perfection and it is conceptually preferable. Moreover, it has the advantage that perfection can be verified for every type of each player. However, in developing the theory, particularly in proving the existence, distributional strategies are easier to work with.

The remainder of this chapter is structured as follows. Section 4.2 describes the model in detail. In section 4.3 distributional perfect equilibrium is introduced and the results related to this concept are provided, which the most important one is the existence result in Theorem 4.3.3 Section 4.4 discusses the relation between distributional perfection and the approach taken in chapter 3. In section 4.5 the concept of distributional perfection is applied to a class of second-price auctions with interdependent values.

\subsection{The model}

Let $\Gamma$ denote the following Bayesian game: There are $n$ players. The set $T_{i}$ of player $i$ 's types is a complete, separable metric space with a metric $d_{T_{i}}$ and the set $A_{i}$ of player $i$ 's actions is a compact metric space with a metric $d_{A_{i}}$. Let $\mathcal{T}_{i}$ and $\mathcal{A}_{i}$ be the induced Borel $\sigma$-fields on $T_{i}$ and $A_{i}$ respectively. Moreover, let $T=\times_{i=1}^{n} T_{i}$ and $A=\times_{i=1}^{n} A_{i}$. Suppose $\mu$ is a probability measure on the product $\sigma$-field $\mathcal{T}=$ $\otimes_{i=1}^{n} \mathcal{T}_{i}$ with marginal probability $\mu_{i}$ on $\mathcal{T}_{i}$ for every player $i$. We assume that each $\mu_{i}$ is a completely mixed probability measure. Recall that a probability measure is completely mixed if it assigns strictly positive weight to every nonempty open set. Player $i$ 's payoff function $\pi_{i}: T \times A \rightarrow \mathbf{R}$ is bounded and jointly measurable.

We impose two more assumptions on Bayesian game $\Gamma$, following Milgrom and Weber [33]. First, the measure $\mu$ is absolutely continuous ${ }^{3}$ with respect to $\hat{\mu}=\times_{i=1}^{n} \mu_{i}$. This property is called Absolutely Continuous Information. Second, the payoffs are equicontinuous, that is for every player $i$ and every $\varepsilon>0$, there is a set $E \in \mathcal{T}$ such that $\mu\left(E^{c}\right)<\varepsilon$ and the family $\left\{\pi_{i}(t, \cdot) \mid t \in E\right\}$ is equicontinuous. ${ }^{4}$ Milgrom and Weber in [33] give detailed explanation of these two assumptions. Absolutely continuous information is a rather weak assumption and it is satisfied when the type spaces are finite or when each player's type is drawn independently from his type space. As an example this assumption rules out the case of a uniform distribution on the diagonal of the unit square. Although the equicontinuity of payoffs is

\footnotetext{
${ }^{3}$ Let $\mu$ and $\nu$ be two measures on a $\sigma$-field $\Sigma$. The measure $\mu$ is absolutely continuous with respect to $\nu$ if for every $G \in \Sigma, \nu(G)=0$ implies $\mu(G)=0$.

${ }^{4} \mathrm{~A}$ family $\mathfrak{F}$ of real functions on the metric space $X$ is equicontinuous if to every $\varepsilon>0$ corresponds a $\delta>0$ such that for every $x, y \in X$ with $d(x, y)<\varepsilon$ we have $|f(x)-f(y)|<\delta$, for every $f \in \mathfrak{F}$.
} 
truly restrictive, it is needed for the existence of distributional equilibrium. However, in the case of finite action spaces the equicontinuity of payoffs holds.

\section{Behavioral and distributional strategy}

A pure strategy for player $i$ in $\Gamma$ is a measurable function $p_{i}: T_{i} \rightarrow A_{i}$ and a behavioral strategy for him is a function $\beta_{i}: T_{i} \times \mathcal{A}_{i} \rightarrow[0,1]$ such that (1) the section function $\beta_{i}\left(t_{i}, \cdot\right): \mathcal{A}_{i} \rightarrow[0,1]$ is a probability measure for every $t_{i} \in T_{i}$, (2) the section function $\beta_{i}(\cdot, B): T_{i} \rightarrow[0,1]$ is measurable for every $B \in \mathcal{A}_{i}$. When player $i$ plays according to the behavioral strategy $\beta_{i}$, for each type $t_{i} \in T_{i}$, he chooses his action according to the probability measure $\beta_{i}\left(t_{i}, \cdot\right)$. A behavioral strategy $\beta_{i}$ for player $i$ is called pure if there is a pure strategy $p_{i}$ for player $i$ such that $\beta_{i}\left(t_{i}, \cdot\right)=D_{p_{i}\left(t_{i}\right)}(\cdot)$ for every type $t_{i} \in T_{i}$ in which $D$ denotes Dirac measure. For simplicity, we denote this pure behavioral strategy by $p_{i}$.

According to Milgrom and Weber [33], a distributional strategy for player $i$ in a Bayesian game is a probability measure $\gamma_{i}$ on $T_{i} \times A_{i}$ such that the marginal distribution of $\gamma_{i}$ on $T_{i}$ equals the probability measure $\mu_{i}$, i.e., $\gamma_{i}\left(U \times A_{i}\right)=\mu_{i}(U)$ for every $U \in \mathcal{T}_{i}$. Given a behavioral strategy $\beta_{i}$, the induced distributional strategy $\gamma_{i}$ is uniquely determined by

$$
\gamma_{i}(U \times B)=\int_{U} \beta_{i}\left(t_{i}, B\right) \mu_{i}\left(d t_{i}\right)
$$

for every rectangle $U \times B \in \mathcal{T}_{i} \otimes \mathcal{A}_{i}$. Conversely, the behavioral strategies corresponding to a distributional strategy $\gamma_{i}$ are exactly the regular conditional probabilities. Hence, there is a many-to-one mapping from behavioral strategies to distributional strategies that preserves the players' ex ante expected payoffs. Notice that in games with finite action and type spaces, if player $i$ receives each type from his type space with strictly positive probability, then the mapping from behavioral strategies to distributional strategies is one to one.

Let $\Delta_{i}$ be the set of all distributional strategies for player $i$ and $\Delta=\times_{i=1}^{n} \Delta_{i}$. Also, let $\Pi_{i}: \Delta \rightarrow \mathbf{R}$ be the expected payoff of player $i$ playing distributional strategy $\gamma_{i}$ against a distributional strategy profile $\gamma_{-i}$, i.e.

$$
\Pi_{i}\left(\gamma_{i}, \gamma_{-i}\right)=\int \pi_{i}(t, a) \gamma_{i}\left(d a_{i} \mid t_{i}\right) \gamma_{-i}\left(d a_{-i} \mid t_{-i}\right) \mu(d t)
$$

where $t=\left(t_{1}, \ldots, t_{n}\right) \in T$ and $a=\left(a_{1}, \ldots, a_{n}\right) \in A, \gamma_{i}\left(d a_{i} \mid t_{i}\right)$ is a version of conditional probability on $A_{i}$ given $t_{i}$, and by integrals with respect to $\gamma_{-i}\left(d a_{-i} \mid\right.$ $\left.t_{-i}\right)$ we mean the iterated integrals with respect to $\gamma_{j}\left(d a_{j} \mid t_{j}\right)$ for all $j \neq i$. As the measure $\mu$ is absolutely continuous with respect to $\hat{\mu}$, the Radon-Nikodym theorem 
implies the existence of a measurable function $f: T \rightarrow \mathbf{R}$ such that for every measurable set $G \in \mathcal{T}$ we have $\mu(G)=\int_{G} f d \hat{\mu}$. Therefore, the expected payoff $\Pi_{i}$ can be expressed in an easier form as

$$
\Pi_{i}\left(\gamma_{i}, \gamma_{-i}\right)=\int \pi_{i}(t, a) f(t) d \gamma_{i} d \gamma_{-i}
$$

Let $\beta_{i}$ be a behavioral strategy corresponding to the distributional strategy $\gamma_{i}$, for every player $i$. Then, by Theorem 10.2.1 in Dudley $[11]^{5}$ we conclude that

$$
\Pi_{i}\left(\gamma_{i}, \gamma_{-i}\right)=\int \pi_{i}(t, a) f(t) \beta_{i}\left(t_{i}, d a_{i}\right) \mu_{i}\left(d t_{i}\right) \beta_{-i}\left(t_{-i}, d a_{-i}\right) \mu_{-i}\left(d t_{-i}\right) .
$$

We use $\Pi_{i}^{b}\left(\beta_{i}, \beta_{-i} \mid t_{i}\right)$ to denote player $i$ 's expected payoff given his behavioral strategy $\beta_{i}$ and his type $t_{i}$ against a behavioral strategy profile $\beta_{-i}$. Thus,

$$
\Pi_{i}^{b}\left(\beta_{i}, \beta_{-i} \mid t_{i}\right)=\int \pi_{i}(t, a) f(t) \beta_{i}\left(t_{i}, d a_{i}\right) \beta_{-i}\left(t_{-i}, d a_{-i}\right) \mu_{-i}\left(d t_{-i}\right) .
$$

One can also define $\Pi_{i}^{b}\left(\sigma_{i}, \beta_{-i} \mid t_{i}\right)$ and $\Pi_{i}^{b}\left(a_{i}, \beta_{-i} \mid t_{i}\right)$ respectively for every probability measure $\sigma_{i}$ on $\mathcal{A}_{i}$ and every action $a_{i} \in A_{i}$. Furthermore, notice that

$$
\Pi_{i}\left(\gamma_{1}, \ldots, \gamma_{n}\right)=\int \Pi_{i}^{b}\left(\beta_{i}, \beta_{-i} \mid t_{i}\right) \mu_{i}\left(d t_{i}\right)
$$

In the special case where player $i$ chooses a pure behavioral strategy $p_{i}$, his expected payoff is

$$
\Pi_{i}^{b}\left(p_{i}, \beta_{-i} \mid t_{i}\right)=\int \pi_{i}\left(t,\left(p_{i}\left(t_{i}\right), a_{-i}\right)\right) f(t) \beta_{-i}\left(t_{-i}, d a_{-i}\right) \mu_{-i}\left(d t_{-i}\right) .
$$

\section{BNE and distributional equilibrium}

A probability measure $\sigma_{i}$ on $\mathcal{A}_{i}$ is called a best response of player $i$ for type $t_{i} \in T_{i}$ against a behavioral strategy profile $\tau_{-i}$, if for every probability measure $\sigma_{i}^{\prime}$ on $\mathcal{A}_{i}$ we have

$$
\Pi_{i}^{b}\left(\sigma_{i}, \tau_{-i} \mid t_{i}\right) \geq \Pi_{i}^{b}\left(\sigma_{i}^{\prime}, \tau_{-i} \mid t_{i}\right)
$$

The set of such best responses is denoted by $B R_{i}\left(t_{i}, \tau\right)$. A behavioral strategy $\beta_{i}$ is called a best response of player $i$ against the behavioral strategy profile $\tau_{-i}$,

${ }^{5}$ Dudley's notation and terminology in [11] differs from ours. Part (II) of Theorem 10.2.1. in our terminology can be expressed as follows: For every measurable function $g: T_{i} \times A_{i} \rightarrow \mathrm{R}$ we have

$$
\int g d \gamma_{i}=\iint g\left(t_{i}, a_{i}\right) \beta_{i}\left(t_{i}, d a_{i}\right) \mu_{i}\left(d t_{i}\right)
$$


if $\beta_{i}\left(t_{i}, \cdot\right) \in B R_{i}\left(t_{i}, \tau\right)$ for every $t_{i} \in T_{i}$. A behavioral strategy profile $\beta=$ $\left(\beta_{1}, \ldots, \beta_{n}\right)$ is a Bayesian Nash Equilibrium $(B N E)$ if $\beta_{i}$ is a best response to $\beta_{-i}$ for every player $i$.

Distributional strategy $\gamma_{i}$ of player $i$ is a best response against a distributional strategy profile $\eta_{-i}$, if $\Pi_{i}\left(\gamma_{i}, \eta_{-i}\right) \geq \Pi_{i}\left(\widetilde{\gamma}_{i}, \eta_{-i}\right)$, for every distributional strategy $\widetilde{\gamma}_{i}$ of player $i$. Define the correspondence $B R_{i}: \Delta \rightarrow \Delta_{i}$ to be the best response correspondence for player $i$ on the set of distributional strategies. Moreover, define $B R: \Delta \rightarrow \Delta$, for every $\gamma \in \Delta$, as $B R(\gamma)=\times_{i=1}^{n} B R_{i}(\gamma)$.

The equilibrium concept in Bayesian games can be defined in a weaker form where each player maximizes his payoff in ex ante probabilities, in other words, by using distributional strategies. This equilibrium concept is used by Milgrom and Weber in [33] and they prove that the Bayesian game $\Gamma$ described above, contains at least one of them. However, it is not clear how players would play the game if they are given their distributional strategies, while behavioral strategies do not have this problem. Hence, we define a behavioral strategy profile $\beta=\left(\beta_{1}, \ldots, \beta_{n}\right)$ to be a distributional equilibrium, if for the induced distributional strategy profile $\gamma=\left(\gamma_{1}, \ldots, \gamma_{n}\right)$ we have $\gamma_{i} \in B R_{i}(\gamma)$, for every player $i$.

Next proposition proves that BNE and distributional equilibrium are equal up to a $\mu_{i}$-measure 1 set of types for every player $i$.

Proposition 4.2.1 For every distributional equilibrium $\beta$, assume that the set of the best responses of player $i$ against behavioral strategy profile $\beta_{-i}$ is not empty. ${ }^{6}$ Then, there is a BNE $\hat{\beta}$ such that for every player $i$ we have $\beta_{i}\left(t_{i}, \cdot\right)=\hat{\beta}_{i}\left(t_{i}, \cdot\right)$, for $\mu_{i}$-a.e. $t_{i} \in T_{i}$

Proof. Let $\beta=\left(\beta_{1}, \ldots, \beta_{n}\right)$ be a distributional equilibrium. Then, for the induced distributional strategy profile $\gamma=\left(\gamma_{1}, \ldots, \gamma_{n}\right)$ we have $\gamma_{i} \in B R_{i}(\gamma)$. Now, by Lemma 4.6.1 we have $\beta_{i}\left(t_{i}, \cdot\right) \in B R_{i}\left(t_{i}, \beta\right)$ for $\mu_{i}$-a.e. $t_{i} \in T_{i}$. In other words, for every $i$ there exists a set $S_{i} \in \mathcal{T}_{i}$ with $\mu_{i}\left(S_{i}\right)=0$ such that for every $t_{i} \in T_{i} \backslash S_{i}$ we have $\beta_{i}\left(t_{i}, \cdot\right) \in B R_{i}\left(t_{i}, \beta\right)$. Now, define $\hat{\beta}=\left(\hat{\beta_{1}}, \ldots, \hat{\beta_{n}}\right)$, for every $t_{i} \in T_{i} \backslash S_{i}$, by letting $\hat{\beta}_{i}\left(t_{i}, \cdot\right)=\beta_{i}\left(t_{i}, \cdot\right)$ and for every $t_{i} \in S_{i}$ selecting any $\hat{\beta}_{i}\left(t_{i}, \cdot\right) \in B R_{i}\left(t_{i}, \beta\right)$. It is clear that $\hat{\beta}$ is a BNE. This completes the proof.

We say a behavioral strategy $\beta_{i}$ (weakly) dominates $\hat{\beta}_{i}$ if for their induced distributional strategies $\gamma_{i}$ and $\hat{\gamma}_{i}$ respectively, we have

$$
\Pi_{i}\left(\gamma_{i}, \gamma_{-i}\right) \geq \Pi_{i}\left(\hat{\gamma}_{i}, \gamma_{-i}\right)
$$

\footnotetext{
${ }^{6}$ The existence of such behavioral strategy might be derived from measurable selection theorems for example in [1] and [46].
} 
for all $\gamma_{-i} \in \Delta_{-i}$, with a strict inequality for some $\gamma_{-i}$. The behavioral strategy $\beta_{i}$ is called (weakly) dominant if it dominates every other behavioral strategy of player $i$. The behavioral strategy $\beta_{i}$ is undominated if there is no other behavioral strategy that dominates $\beta_{i}$.

\subsection{Distributional perfection}

We define perfect equilibrium for Bayesian games in behavioral strategies, because a player given a behavioral strategy knows how to play the game, but this is not clear if he is given a distributional strategy. Nevertheless, we use distributional strategies as a tool to verify whether the behavioral strategy profile satisfies the conditions of trembling hand perfect equilibrium or not. This concept is called distributional perfect equilibrium. Throughout this chapter the weak metric (also called Prohorov metric) on a set of probability measures is denoted by $\rho^{w}$.

Definition 4.3.1 A behavioral strategy profile $\beta=\left(\beta_{1}, \ldots, \beta_{n}\right)$ is called distributional perfect if for the induced distributional strategy profile $\gamma=\left(\gamma_{1}, \ldots, \gamma_{n}\right)$ there exists a sequence of completely mixed distributional strategy profiles $\left(\gamma^{k}\right)_{k=1}^{\infty}=$ $\left(\gamma_{1}^{k}, \ldots, \gamma_{n}^{k}\right)_{k=1}^{\infty}$ such that for every player $i$ :

(i) $\lim _{k \rightarrow \infty} \rho^{w}\left(\gamma_{i}^{k}, \gamma_{i}\right)=0$,

(ii) $\lim _{k \rightarrow \infty} \rho^{w}\left(\gamma_{i}^{k}, B R_{i}\left(\gamma^{k}\right)\right)=0$.

Distributional perfection in Bayesian game $\Gamma$ is a refinement of distributional equilibrium. This is proved in the following theorem.

Theorem 4.3.2 Every distributional perfect strategy profile in Bayesian game $\Gamma$, is a distributional equilibrium.

Proof. Let $\beta=\left(\beta_{1}, \ldots, \beta_{n}\right)$ be a distributional perfect strategy profile. By Definition 4.3.1, for the induced distributional strategy profile $\gamma=\left(\gamma_{1}, \ldots, \gamma_{n}\right)$ there exists a sequence of completely mixed distributional strategy profiles $\left(\gamma^{k}\right)_{k=1}^{\infty}=$ $\left(\gamma_{1}^{k}, \ldots, \gamma_{n}^{k}\right)_{k=1}^{\infty}$ such that for every player $i$ we have $\lim _{k \rightarrow \infty} \rho^{w}\left(\gamma_{i}^{k}, \gamma_{i}\right)=0$ and $\lim _{k \rightarrow \infty} \rho^{w}\left(\gamma_{i}^{k}, B R_{i}\left(\gamma^{k}\right)\right)=0$. Therefore, for every $i$, there exists a sequence $\left(\hat{\gamma}_{i}^{k}\right)_{k=1}^{\infty}$ such that $\hat{\gamma}_{i}^{k} \in B R_{i}\left(\gamma^{k}\right)$ for every $k \in \mathbf{N}$ and $\rho^{w}\left(\gamma_{i}^{k}, \hat{\gamma}_{i}^{k}\right) \rightarrow 0$ as $k \rightarrow \infty$. By the triangle inequality for $\rho^{w}$ this implies for every $i$ that $\rho^{w}\left(\gamma_{i}, \hat{\gamma}_{i}^{k}\right) \rightarrow 0$ as $k \rightarrow \infty$. Now, by Lemma 4.6.3 we conclude the upper hemicontinuity of the correspondence $B R_{i}$, for every player $i$. Hence, $\gamma_{i} \in B R_{i}(\gamma)$, which implies that behavioral strategy $\beta$ is distributional perfect. 
As we just proved, distributional perfection is a refinement of distributional equilibrium, but it may not be a refinement of BNE. In other words, distributional perfection does not guarantee that player $i$, for every type $t_{i} \in T_{i}$, selects a best response against the opponents' strategies. However, by Proposition 4.2.1, given the distributional perfect equilibrium, we can construct a BNE that satisfies conditions (i) and (ii) of Definition 4.3.1 as follows. Let $\beta$ be a distributional perfect strategy profile. Then, if for any type $t_{i}$ of player $i, \beta_{i}\left(t_{i}, \cdot\right)$ is not a best response against $\beta_{-i}$, he moves to one of his best responses, while this does not change the expected payoffs of the opponents, since it happens only on a measure zero set.

In addition, we prove the existence of distributional perfect BNE in Bayesian game $\Gamma$ and that the set of distributional perfect equilibria is sequentially closed in the topology $\omega$, where $\omega$ is the Tychonoff topology on the set $\times_{t_{i} \in T_{i}} \Delta\left(A_{i}\right)$ when taking the strong topology on each $\Delta\left(A_{i}\right)$.

Theorem 4.3.3 In Bayesian game $\Gamma$, the set of distributional perfect equilibria is nonempty and sequentially closed in the topology $\omega$.

Proof. First we prove that the set of distributional strategy profiles satisfying conditions (i) and (ii) of Definition 4.3.1 is not empty. For every player $i$, let $\nu_{i}$ be a completely mixed distributional strategy. Note that such a $\nu_{i}$ exists, because $T_{i} \times A_{i}$ is separable. Define for every $k \in \mathbf{N}$

$$
\Delta_{i}(k)=\left\{\gamma_{i} \in \Delta_{i} \mid \gamma_{i}\left(B_{i}\right) \geq \frac{1}{k} \cdot \nu_{i}\left(B_{i}\right), \quad \forall B_{i} \in \mathcal{T}_{i} \otimes \mathcal{A}_{i}\right\}
$$

Moreover, let $\Delta(k)=\times_{i=1}^{n} \Delta_{i}(k)$ and $B R_{i}^{k}: \Delta(k) \rightarrow \Delta_{i}(k)$ be the best response correspondence for player $i$ restricted to $\Delta_{i}(k)^{7}$ and define the correspondence $B R^{k}: \Delta(k) \rightarrow \Delta(k)$ to be

$$
B R^{k}(\gamma)=\times_{i=1}^{n} B R_{i}^{k}(\gamma)
$$

for every $\gamma \in \Delta(k)$. We verify the conditions of Theorem 4.6.7 for the correspondence $B R^{k}$.

Since $T$ is a complete and separable metric space, by Theorem 1.4 in [6] $\mu$ is a tight measure. ${ }^{8}$ This fact together with the compactness of the set $A$, implies that $\Delta_{i}$

\footnotetext{
${ }^{7}$ Distributional strategy $\gamma_{i}$ of player $i$ is a best response restricted to $\Delta_{i}(k)$ against a distributional strategy profile $\eta_{-i} \in \Delta_{-i}(k)$, if $\Pi_{i}\left(\gamma_{i}, \eta_{-i}\right) \geq \Pi_{i}\left(\widetilde{\gamma}_{i}, \eta_{-i}\right)$, for every distributional strategy $\widetilde{\gamma}_{i} \in$ $\Delta_{i}(k)$ of player $i$.

${ }^{8}$ Let $X$ be a metric space with the Borel $\sigma$-field $\Sigma$. A measure $\mu$ on $\Sigma$ is tight if for every $G \in \Sigma$, $\mu(G)=\sup \{\mu(K) \mid K \subset G$, and K compact $\}$. Moreover, Theorem 1.4 in [6] states that if $X$ is separable and complete, then each probability measure on $\Sigma$ is tight.
} 
is a tight set of probability measures. ${ }^{9}$ Now, by Prohorov's Theorem we conclude that $\Delta_{i}$ is a compact metric space with respect to the weak metric. It is easy to see that $\Delta_{i}(k)$ is a closed subset of $\Delta_{i}$ with respect to the strong metric. Consequently, by Theorem V.3.13 in [12], it is closed with respect to the weak metric. Hence, $\Delta_{i}(k)$ is compact with respect to the weak metric. Also, one can easily check that $\Delta_{i}(k)$ is convex. Moreover, upper hemicontinuity of $B R^{k}$ follows by Lemma 4.6.3. Now, we apply Theorem 4.6.7, which leads us to the existence of an equilibrium point $\gamma^{k} \in \Delta(k)$, for every $k$.

Now, define $e^{10}$

$$
P B R_{i}(\gamma)=\left\{\left(t_{i}, a_{i}\right) \in T_{i} \times A_{i} \mid\left(t_{i}, a_{i}\right) \in \operatorname{supp}\left(\gamma_{i}\right) \text { and } \gamma_{i} \in B R_{i}(\gamma)\right\}
$$

It is clear that $\gamma_{i}^{k}\left(P B R_{i}\left(\gamma^{k}\right)\right) \geq 1-\frac{1}{k} \nu_{i}\left(T_{i} \times A_{i}\right)=1-\frac{1}{k}$. This implies that $\rho^{w}\left(\gamma_{i}^{k}, B R_{i}\left(\gamma^{k}\right)\right) \leq \frac{1}{k}$. Furthermore, as $\Delta_{i}$ is compact with respect to the weak metric, without loss of generality we can assume that there is a distributional strategy profile $\gamma$ such that $\rho^{w}\left(\gamma^{k}, \gamma\right) \rightarrow 0$ when $k \rightarrow \infty$. Let $\beta$ be a behavioral strategy profile corresponding to $\gamma$. Obviously, $\beta$ is a distributional perfect equilibrium. Hence, the set of distributional perfect equilibria is nonempty.

Now, we prove that the set of distributional perfect equilibria is sequentially closed in $\omega$. Let $\left(\beta_{\ell}\right)_{\ell=1}^{\infty}$ be a sequence of distributional perfect equilibria in which $\beta_{\ell}=\left(\beta_{\ell, 1}, \ldots, \beta_{\ell, n}\right)$ and suppose that for every player $i$, the behavioral strategy $\beta_{\ell, i}$ converges to $\beta_{i} \in \times_{t_{i} \in T_{i}} \Delta\left(A_{i}\right)$ in the topology $\omega$ as $\ell \rightarrow \infty$. Then, for every $B \in \mathcal{A}_{i}$ the function $\beta_{\ell, i}(\cdot, B)$ on $T_{i}$ converges pointwisely to $\beta_{i}(\cdot, B)$ as $\ell \rightarrow \infty$. This implies that $\beta_{i}(\cdot, B)$ is measurable for every $B \in \mathcal{A}_{i}$, hence $\beta_{i}$ is a behavior strategy. Let $\beta=\left(\beta_{1}, \ldots, \beta_{n}\right)$. We show that $\beta$ is distributional perfect. Let $\gamma_{\ell}=$ $\left(\gamma_{\ell, 1}, \ldots, \gamma_{\ell, n}\right)$ and $\gamma=\left(\gamma_{1}, \ldots, \gamma_{n}\right)$ be the induced distributional strategy profiles by $\beta_{\ell}$ and $\beta$ respectively. It is clear that $\rho^{w}\left(\gamma_{\ell, i}, \gamma_{i}\right) \rightarrow 0$ as $\ell \rightarrow \infty$, for every $i$. Since for every $\ell$ the strategy profile $\beta_{\ell}$ is distributional perfect, there exits a sequence of completely mixed distributional strategies $\left(\gamma_{\ell, i}^{k}\right)_{k=1}^{\infty}$, for every player $i$, satisfying the conditions of Definition 4.3.1. Hence, there is a $K_{\ell, i}^{1}$ such that for every $k \geq K_{\ell, i}^{1}$ we have $\rho^{w}\left(\gamma_{\ell, i}^{k}, \gamma_{\ell, i}\right)<\frac{1}{\ell}$. Similarly, there is a $K_{\ell, i}^{2}$ such that for every $k \geq K_{\ell, i}^{2}$ we have $\rho^{w}\left(\gamma_{\ell, i}^{k}, B R_{i}\left(\gamma_{\ell}^{k}\right)\right)<\frac{1}{\ell}$. Let $K_{\ell, i}=\max \left\{K_{\ell, i}^{1}, K_{\ell, i}^{2}\right\}$. Now, the sequence $\left(\gamma_{\ell, i}^{K_{\ell, i}}\right)_{\ell=1}^{\infty}$ of completely mixed distributional strategies satisfies the conditions of Definition 4.3.1 for the strategy profile $\gamma$ when $\ell \rightarrow \infty$. Namely,

\footnotetext{
${ }^{9} \mathrm{~A}$ family of probability measures on a metric space is tight if for every $\varepsilon>0$ there is a compact set $K$ satisfying $\mu(K)>1-\varepsilon$, for every $\mu$ in the family.

${ }^{10}$ Recall that $\operatorname{supp}(\mu)=\{x \in X \mid$ for everey open set $G$ if $x \in G$ then $\mu(G)>0\}$.
} 
for every player $i$ we have

$$
\rho^{w}\left(\gamma_{\ell, i}^{K_{\ell, i}}, \gamma_{i}\right) \leq \rho^{w}\left(\gamma_{\ell, i}^{K_{\ell, i}}, \gamma_{\ell, i}\right)+\rho^{w}\left(\gamma_{\ell, i}, \gamma_{i}\right)<\frac{1}{\ell}+\rho^{w}\left(\gamma_{\ell, i}, \gamma_{i}\right)
$$

and

$$
\rho^{w}\left(\gamma_{\ell, i}^{K_{\ell, i}}, B R_{i}\left(\gamma_{\ell}^{K_{\ell, i}}\right)\right)<\frac{1}{\ell}
$$

Clearly, both $\rho^{w}\left(\gamma_{\ell, i}^{K_{\ell, i}}, \gamma_{i}\right)$ and $\rho^{w}\left(\gamma_{\ell, i}^{K_{\ell, i}}, B R_{i}\left(\gamma_{\ell}^{K_{\ell, i}}\right)\right)$ converge to zero when $\ell \rightarrow \infty$. Hence, $\beta$ is a distributional perfect equilibrium.

In the theorem above, to define $\omega$ we use the strong topology on each $\Delta\left(A_{i}\right)$, but not the weak topology. In the weak topology the limit $\beta_{i}$ of a sequence of behavioral strategies $\left(\beta_{\ell, i}\right)_{\ell=1}^{\infty}$ may not be a behavioral strategy, as we encounter difficulties to show that $\beta_{i}(\cdot, B): T_{i} \rightarrow[0,1]$ is measurable for every $B \in \mathcal{A}_{i}$.

As we explained in the introduction, for finite games perfect equilibrium puts weight zero on dominated strategies. However, this property is not compatible for infinite games, since Simon and Stinchcombe [42] in Example 2.1 presents a game with infinite action spaces in which the unique equilibrium of the game is in weakly dominated strategies. Hence, a weaker property of limit undominatedness is used for strategies in infinite games that is defined as follows: Behavioral strategy $\beta_{i}$ is limit undominated if for the induced distributional strategy $\gamma_{i}$, there is a sequence of undominated ${ }^{11}$ distributional strategies $\left(\gamma_{i}^{k}\right)_{k=1}^{\infty}$ such that $\rho^{w}\left(\gamma_{i}^{k}, \gamma_{i}\right) \rightarrow 0$ as $k \rightarrow \infty$. The following result shows that a distributional perfect strategy profile is in limit undominated strategies. The proof is simple and similar to the proof of Theorem 2.4.1 in chapter 2.

Theorem 4.3.4 Let $\beta$ be a distributional perfect equilibrium in Bayesian game $\Gamma$. Then, $\beta_{i}$ is limit undominated, for every player $i$.

In the remark below we see that distributional perfection is equivalent to trembling hand perfect equilibrium in games with finite action and type spaces.

Remark. Consider a Bayesian game in which every player's action space and type space are finite and every player receives each type from his type space with strictly positive probability. This game satisfies all the assumptions of the game $\Gamma$ described above, so it is an special case of this class of Bayesian games. A behavioral strategy for player $i$ in finite games is a function $\beta_{i}: T_{i} \rightarrow \Delta\left(A_{i}\right)$, where $\Delta\left(A_{i}\right)$ is

\footnotetext{
${ }^{11}$ Undominated distributional strategies are defined similarly to undominated strategies for normal form games, since distributional strategies are simply probability measures on the product space of types and actions.
} 
the set of mixed strategies on $A_{i}$. Behavioral strategy $\beta_{i}$ is called completely mixed if $\beta_{i}\left(t_{i}\right)$ is a completely mixed strategy for each type $t_{i} \in T_{i}$. A direct generalization of Selten's perfect equilibrium to finite Bayesian games in chapter 3 is defined as a behavioral strategy profile $\beta=\left(\beta_{1}, \ldots, \beta_{n}\right)$ for which there exists a sequence of completely mixed behavioral strategy profiles $\left(\beta^{k}\right)_{k=1}^{\infty}=\left(\beta_{1}^{k}, \ldots, \beta_{n}^{k}\right)_{k=1}^{\infty}$ with the following properties for every player $i$ and each type $t_{i} \in T_{i}$ :

(i) $\lim _{k \rightarrow \infty} d_{E}\left(\beta_{i}^{k}\left(t_{i}\right), \beta_{i}\left(t_{i}\right)\right)=0$,

(ii) $\lim _{k \rightarrow \infty} d_{E}\left(\beta_{i}^{k}\left(t_{i}\right), B R_{i}\left(t_{i}, \beta^{k}\right)\right)=0$,

where $d_{E}$ is the Euclidean metric.

Now, define $A_{i}^{\prime}=T_{i} \times A_{i}$ and $\Delta\left(A_{i}^{\prime}\right)$ to be the set of mixed strategies on $A_{i}^{\prime}$. Let $\Delta_{d}\left(A_{i}^{\prime}\right) \subseteq \Delta\left(A_{i}^{\prime}\right)$ to be the set of all player $i$ 's distributional strategies. Moreover, the player $i$ 's payoff is his ex ante expected payoff, i.e. his expected payoff before he learns his own type. The equivalent definition of distributional perfection in finite Bayesian games is:

A behavioral strategy profile $\beta=\left(\beta_{1}, \ldots, \beta_{n}\right)$ is called perfect if for the induced distributional strategy profile $\sigma=\left(\sigma_{1}, \ldots, \sigma_{n}\right)$, there exists a sequence of completely mixed distributional strategy profiles $\left(\sigma^{k}\right)_{k=1}^{\infty}=\left(\sigma_{1}^{k}, \ldots, \sigma_{n}^{k}\right)_{k=1}^{\infty}$ such that for every player $i$ we have:

(i) $\lim _{k \rightarrow \infty} d_{E}\left(\sigma_{i}^{k}, \sigma_{i}\right)=0$,

(ii) $\lim _{k \rightarrow \infty} d_{E}\left(\sigma_{i}^{k}, B R_{i}\left(\sigma^{k}\right)\right)=0$,

In this finite game, for every distributional strategy there is a unique behavioral strategy and vice versa. Also, for a behavioral strategy profile $\beta=\left(\beta_{1}, \ldots, \beta_{n}\right)$ if we have $\beta_{i}\left(t_{i}\right) \in B R_{i}\left(t_{i}, \beta\right)$ for every $t_{i} \in T_{i}$, then obviously for the induced distributional strategy $\gamma=\left(\gamma_{1}, \ldots, \gamma_{n}\right)$ we have $\gamma_{i} \in B R_{i}(\gamma)$, for every player $i$. Conversely, it is easy to see that if $\gamma_{i} \in B R_{i}(\gamma)$, then $\beta_{i}\left(t_{i}, \cdot\right) \in B R_{i}\left(t_{i}, \beta\right)$, for every $t_{i} \in T_{i}$ and every player $i$. Therefore, the latter definition is equivalent to the perfection defined in chapter 3 for finite games.

\subsection{A.e. pointwise perfection}

In chapter 3 we introduced a definition of perfect equilibrium in Bayesian games without using distributional strategies. It is called a.e.-pointwise perfection and it has a close relation with distributional perfection. Recall that a behavioral strategy $\beta_{i}$ is 
completely mixed if the probability measure $\beta_{i}\left(t_{i}, \cdot\right): \mathcal{A}_{i} \rightarrow[0,1]$ is completely mixed, for every $t_{i} \in T_{i}$. A behavioral strategy profile $\beta=\left(\beta_{1}, \ldots, \beta_{n}\right)$ is a.e.pointwise perfect if for every player $i$ there exists a set $S_{i} \in \mathcal{T}_{i}$ of $\mu_{i}$-measure 0 and a sequence of profiles of completely mixed behavioral strategies $\left(\beta^{k}\right)_{k=1}^{\infty}=$ $\left(\beta_{1}^{k}, \ldots, \beta_{n}^{k}\right)_{k=1}^{\infty}$ with the following properties for every player $i$ and every type $t_{i} \in T_{i} \backslash S_{i}$ :

(i) $\lim _{k \rightarrow \infty} \rho^{w}\left(\beta_{i}^{k}\left(t_{i}, \cdot\right), \beta_{i}\left(t_{i}, \cdot\right)\right)=0$,

(ii) $\lim _{k \rightarrow \infty} \rho^{w}\left(\beta_{i}^{k}\left(t_{i}, \cdot\right), B R_{i}\left(t_{i}, \beta^{k}\right)\right)=0$.

In the next proposition we show that a.e.-pointwise perfection is a stronger concept than distributional perfect equilibrium. The opposite direction remains an open question.

Proposition 4.4.1 Every a.e.-pointwise perfect strategy profile is distributional perfect.

Proof. Let $\beta=\left(\beta_{1}, \ldots, \beta_{n}\right)$ be an a.e.-pointwise perfect strategy profile. Then, by the definition, there is a set $S_{i} \in \mathcal{T}_{i}$ of $\mu_{i}$-measure 0 and a sequence of completely mixed profiles of behavioral strategies $\left(\beta^{k}\right)_{k=1}^{\infty}=\left(\beta_{1}^{k}, \ldots, \beta_{n}^{k}\right)_{k=1}^{\infty}$ such that for every player $i$ and every type $t_{i} \in T_{i} \backslash S_{i}$ conditions (i) and (ii) of this definition are satisfied. For every player $i$, let $\gamma_{i}$ and $\gamma_{i}^{k}$ be the distributional strategies induced by $\beta_{i}$ and $\beta_{i}^{k}$ respectively. We prove that conditions (i) and (ii) of Definition 4.3.1 are satisfied. Take a player $i$. For condition (i), we show that for any bounded and continuous function $h: T_{i} \times A_{i} \rightarrow \mathbf{R}$, we have

$$
\int_{T_{i} \times A_{i}} h d \gamma_{i}^{k} \longrightarrow \int_{T_{i} \times A_{i}} h d \gamma_{i} \text { as } k \rightarrow \infty .
$$

As $h$ is bounded and continuous on $T_{i} \times A_{i}$, the function $h_{t_{i}}\left(a_{i}\right)=h\left(t_{i}, a_{i}\right)$ on $A_{i}$ is also bounded and continuous for every $t_{i} \in T_{i}$. Let

$$
g^{k}\left(t_{i}\right)=\int_{A_{i}} h_{t_{i}} \beta_{i}^{k}\left(t_{i}, d a_{i}\right), g\left(t_{i}\right)=\int_{A_{i}} h_{t_{i}} \beta_{i}\left(t_{i}, d a_{i}\right) .
$$

By Theorem 10.2.1 in [11], the functions $g^{k}$ and $g$ are measurable. Moreover, because for every type $t_{i} \in T_{i} \backslash S_{i}, \lim _{k \rightarrow \infty} \rho^{w}\left(\beta_{i}^{k}\left(t_{i}, \cdot\right), \beta_{i}\left(t_{i}, \cdot\right)\right)=0$, we have $g^{k}\left(t_{i}\right) \longrightarrow g\left(t_{i}\right)$ as $k \rightarrow \infty$, for $\mu_{i}$-a.e. $t_{i} \in T_{i}$. Therefore, by Dominated Convergence Theorem

$$
\int_{T_{i}} g^{k}\left(t_{i}\right) \mu_{i}\left(d t_{i}\right) \longrightarrow \int_{T_{i}} g\left(t_{i}\right) \mu_{i}\left(d t_{i}\right) \text { as } k \rightarrow \infty .
$$


This implies that

$$
\int_{T_{i} \times A_{i}} h d \gamma_{i}^{k} \longrightarrow \int_{T_{i} \times A_{i}} h d \gamma_{i} \text { as } k \rightarrow \infty .
$$

Now, we prove that condition (ii) of Definition 4.3.1 is satisfied. We know that for every $t_{i} \in T_{i} \backslash S_{i}$ we have $\rho^{w}\left(\beta_{i}^{k}\left(t_{i}, \cdot\right), B R_{i}\left(t_{i}, \beta^{k}\right)\right) \rightarrow 0$ as $k \rightarrow \infty$. Hence, for every $t_{i} \in T_{i} \backslash S_{i}$, there exists a sequence $\hat{\beta}_{i}^{k}\left(t_{i}, \cdot\right) \in B R_{i}\left(t_{i}, \beta^{k}\right)$ such that $\rho^{w}\left(\beta_{i}^{k}\left(t_{i}, \cdot\right), \hat{\beta}_{i}^{k}\left(t_{i}, \cdot\right)\right) \rightarrow 0$ as $k \rightarrow \infty$. Define $\hat{\gamma}_{i}^{k}$ to be the distributional strategies induced by $\hat{\beta}_{i}^{k}$ for every $k \in \mathbf{N}$. With the same argument as in the previous part, we conclude that $\rho^{w}\left(\gamma_{i}^{k}, \hat{\gamma}_{i}^{k}\right) \rightarrow 0$ as $k \rightarrow \infty$. Since

$$
\Pi_{i}\left(\gamma_{i}, \gamma_{-i}\right)=\int_{T_{i}} \Pi_{i}^{b}\left(\beta_{i}, \beta_{-i} \mid t_{i}\right) \mu_{i}\left(d t_{i}\right),
$$

one can easily verify that if $\hat{\beta}_{i}^{k}\left(t_{i}, \cdot\right) \in B R_{i}\left(t_{i}, \beta^{k}\right)$ for every $t_{i} \in T_{i} \backslash S_{i}$, then $\hat{\gamma}_{i}^{k} \in B R_{i}\left(\gamma^{k}\right)$. Hence, we have $\lim _{k \rightarrow \infty} \rho^{w}\left(\gamma_{i}^{k}, B R_{i}\left(\gamma_{i}^{k}\right)\right)=0$. So, condition (ii) holds.

\subsection{Second-price auctions with interdependent values}

In this section we study symmetric second-price auctions with interdependent values for two bidders and apply distributional perfection in this context. Let $\Lambda$ be the following sealed-bid second-price auction for a single indivisible object. There are 2 bidders. Prior to bidding, each bidder $i$ receives a private signal $t_{i} \in T_{i}=[\underline{t}, \bar{t}]$, which is called the type of bidder $i$. Signals are drawn independently according to a distribution $\mu_{i}$. Bidder $i$ 's valuation of the object may depend on both types and is denoted by $v_{i}\left(t_{1}, t_{2}\right)$. Each bidder $i$, after observing his own type, submits a bid from a set $A_{i}$ independently of his opponent, where $A_{i}$ is sufficiently large in the sense that it contains the range of $v_{i}$. Given the bids submitted by the bidders, the highest bid wins the auction for the price equal to the second highest bid. ${ }^{12}$ Suppose the following assumptions in this auction:

(1) $v_{1}\left(t_{1}, t_{2}\right)=v_{2}\left(t_{2}, t_{1}\right)$.

(2) $v_{i}$ is continuously differentiable.

(3) $v_{i}$ is strictly increasing in $t_{i}$ and increasing in $t_{j}$ for $j \neq i$.

In $\Lambda$ the set of available bids for bidder $i$ is an interval $A_{i}=[\underline{a}, \bar{a}]$, where $v_{i}(\underline{t}, \underline{t})=\underline{a}$ and $v_{i}(\bar{t}, \bar{t})=\bar{a}$, for $i=1,2$. In such auctions bidders do not necessarily have any dominant strategy, however it may contain many equilibria. Hence, a

\footnotetext{
${ }^{12}$ In the event of a tie for the highest bid, the winner is chosen according to a probability distribution which may depend on the identity of the highest bidder and his type as well. In our setting, a tie will only occur with probability 0 , even given the type of one bidder, which makes the specification of this tie-breaking rule irrelevant for the expected profits.
} 
refinement is needed in order to rule out the less intuitive equilibria and get a smaller set of solutions. We use distributional perfection for this purpose. In Proposition 4.5.1 we show that a class of BNEs that was discovered by Milgrom in [32] are distributional perfect and in Proposition 4.5.3 we prove that distributional perfection rules out another class of equilibria introduced by Krishna in [23].

Proposition 4.5.1 Let $\phi:[\underline{t}, \bar{t}] \rightarrow[\underline{t}, \bar{t}]$ be an increasing bijection. Consider the strategy profile $\left(b_{1}, b_{2}\right)$ in which $b_{1}\left(t_{1}\right)=v_{1}\left(t_{1}, \phi\left(t_{1}\right)\right)$ and $b_{2}\left(t_{2}\right)=v_{2}\left(\phi^{-1}\left(t_{2}\right), t_{2}\right)$. If $v_{i}(t, \phi(t))=v_{i}(\phi(t), t)$, for $i=1,2$, then $\left(b_{1}, b_{2}\right)$ is a distributional perfect $B N E$. Moreover, $b_{i}$ is undominated for every bidder $i$.

Proof. First we show that $\left(b_{1}, b_{2}\right)$ is a BNE. Define $w(t)=v_{1}(t, \phi(t))=$ $v_{2}(\phi(t), t)$ for every $t \in[\underline{t}, \bar{t}]$. One can easily see that $w:[\underline{t}, \bar{t}] \rightarrow[\underline{a}, \bar{a}]$ is an increasing bijection. Suppose that bidder 1 bids $b_{1}\left(t_{1}\right)=v_{1}\left(t_{1}, \phi\left(t_{1}\right)\right)$. The expected payoff of bidder 2 having type $t_{2}$ and bidding $p_{2}$ is:

$$
\Pi_{2}^{b}\left(b_{1}, p_{2} \mid t_{2}\right)=\int_{\underline{t}}^{w^{-1}\left(p_{2}\right)}\left(v_{2}\left(t_{1}, t_{2}\right)-v_{1}\left(t_{1}, \phi\left(t_{1}\right)\right)\right) \mu_{1}\left(d t_{1}\right) .
$$

As $v_{2}$ is strictly increasing in the second argument and we know

$$
v_{2}\left(t_{1}, t_{2}\right)-v_{1}\left(t_{1}, \phi\left(t_{1}\right)\right)=v_{2}\left(t_{1}, t_{2}\right)-v_{2}\left(\phi\left(t_{1}\right), t_{1}\right)=v_{2}\left(t_{1}, t_{2}\right)-v_{2}\left(t_{1}, \phi\left(t_{1}\right)\right),
$$

it is clear that if $\phi\left(t_{1}\right) \leq t_{2}$, then $v_{2}\left(t_{1}, t_{2}\right)-v_{1}\left(t_{1}, \phi\left(t_{1}\right)\right) \geq 0$ and negative otherwise. Hence, the maximum of $\Pi_{i}^{b}\left(b_{1}, p_{2} \mid t_{2}\right)$ is obtained by choosing $p_{2}$ such that $w^{-1}\left(p_{2}\right)=t_{1}=\phi^{-1}\left(t_{2}\right)$ which means $p_{2}=w\left(\phi^{-1}\left(t_{2}\right)\right)=v_{2}\left(t_{2}, \phi^{-1}\left(t_{2}\right)\right)=$ $b_{2}\left(t_{2}\right)$. Furthermore, $\Pi_{2}^{b}\left(b_{1}, p_{2} \mid t_{2}\right)$ is maximized over $p_{2}$ if and only if $p_{2}=b_{2}\left(t_{2}\right)$, which implies that bidding $b_{2}$ is the unique best response against $b_{1}$ for bidder 2 .

Next, we prove that the strategy profile $\left(b_{1}, b_{2}\right)$ is distributional perfect. Let $\gamma_{1}$ be the distributional strategy induced by $b_{1}$ and for every $k \in \mathbf{N}$ define $\gamma_{1}^{k}=$ $\left(1-\varepsilon_{k}\right) \gamma_{1}+\varepsilon_{k} \eta_{1}$, where $\varepsilon_{k} \in(0,1), \varepsilon_{k} \rightarrow 0$ as $k \rightarrow \infty$, and $\eta_{1}$ is the normalized uniform distributional strategy ${ }^{13}$ on the product space $T_{1} \times A_{1}$. Let $p_{2}$ be a pure behavioral strategy for bidder 2 and $\sigma_{2}$ be the induced distributional strategy by $p_{2}$. Then, the expected payoff of bidder 2 choosing $\sigma_{2}$ against $\gamma_{1}^{k}$ is:

$$
\begin{aligned}
\Pi_{2}\left(\gamma_{1}^{k}, \sigma_{2}\right) & =\iint_{p_{2}>a_{1}}\left(v_{2}\left(t_{1}, t_{2}\right)-a_{1}\right) d \gamma_{1}^{k} d \sigma_{2} \\
& =\int_{T_{2}} \int_{p_{2}>a_{1}}\left(v_{2}\left(t_{1}, t_{2}\right)-a_{1}\right) d \gamma_{1}^{k} \mu_{2}\left(d t_{2}\right) \\
& =\int_{T_{2}} \Pi_{2}^{b}\left(\beta_{1}^{k}, p_{2} \mid t_{2}\right) \mu_{2}\left(d t_{2}\right),
\end{aligned}
$$

${ }^{13}$ That is $\eta_{1}\left(S_{1} \times A_{1}\right)=\mu_{1}\left(S_{1}\right)$ for every $S_{1} \in \mathcal{T}_{1}$ and for a behavioral strategy $\lambda_{1}$ corresponding to $\eta_{1}$ we have $\lambda_{1}\left(t_{1}, \cdot\right)$ is the uniform distribution on $A_{1}$ for every $t_{1} \in T_{1}$. 
where $\beta_{1}^{k}$ is a behavioral strategy corresponding to $\gamma_{1}^{k}$ for every $k \in \mathbf{N}$. To maximize $\Pi_{2}\left(\gamma_{1}^{k}, \sigma_{2}\right)$ it is enough to maximize $\Pi_{2}^{b}\left(\beta_{1}^{k}, p_{2} \mid t_{2}\right)$ over $p_{2}$. We have

$$
\begin{aligned}
\Pi_{2}^{b}\left(\beta_{1}^{k}, p_{2} \mid t_{2}\right) & =\left(1-\varepsilon_{k}\right) \int_{p_{2}>a_{1}}\left(v_{2}\left(t_{1}, t_{2}\right)-a_{1}\right) d \gamma_{1} \\
& +\varepsilon_{k} \int_{p_{2}>a_{1}}\left(v_{2}\left(t_{1}, t_{2}\right)-a_{1}\right) d \eta_{1} \\
& =\left(1-\varepsilon_{k}\right) \int_{\underline{t}}^{w^{-1}\left(p_{2}\right)}\left(v_{2}\left(t_{1}, t_{2}\right)-v_{1}\left(t_{1}, \phi\left(t_{1}\right)\right)\right) \mu_{1}\left(d t_{1}\right) \\
& +\varepsilon_{k} \int_{p_{2}>a_{1}}\left(v_{2}\left(t_{1}, t_{2}\right)-a_{1}\right) d \eta_{1} \\
& =\left(1-\varepsilon_{k}\right) \Pi_{2}^{b}\left(b_{1}, p_{2} \mid t_{2}\right)+\varepsilon_{k} \int_{p_{2}>a_{1}}\left(v_{2}\left(t_{1}, t_{2}\right)-a_{1}\right) d \eta_{1}
\end{aligned}
$$

Suppose that $p_{2}=b_{2}^{k}\left(t_{2}\right)$ maximizes $\Pi_{2}^{b}\left(\beta_{1}^{k}, p_{2} \mid t_{2}\right)$ for every $k$. Also, we know that $p_{2}=b_{2}$ is the unique maximizer of $\Pi_{2}^{b}\left(b_{1}, p_{2} \mid t_{2}\right)$. Then, clearly we have that $b_{2}^{k}$ converges to $b_{2}$ when $k \rightarrow \infty$. With a similar argument for bidder 2 , we can conclude that $\left(b_{1}, b_{2}\right)$ is distributional perfect.

Finally, the fact that bidding $b_{2}$ is the unique best response for bidder 2 against $b_{1}$, implies that $b_{2}$ is undominated. A similar argument holds for $b_{1}$.

Remark that if for the class of strategy profiles $\left(b_{1}, b_{2}\right)$ in Proposition 4.5.1 one can find more than one map $\phi$ that satisfies the condition $v_{i}(t, \phi(t))=v_{i}(\phi(t), t)$, for $i=1,2$, then obviously these strategy profiles are not in dominant strategies. Because, for each $\phi, b_{i}$ the unique best response for bidder $i$ against $b_{j}$ where $j \neq i$. In the case that this class of equilibria only contains one strategy profile, that would be the equilibrium in which $b_{i}=v_{i}\left(t_{i}, t_{i}\right)$ for every $i=1,2$. In the next proposition we prove that this strategy profile is not in dominant strategies.

Proposition 4.5.2 Suppose that there is a $y \in T_{j}$ such that $\frac{\partial v_{i}\left(\bar{t}, t_{j}\right)}{\partial t_{j}}(y)>0$, where $j \neq i$. Then, $b_{i}=v_{i}\left(t_{i}, t_{i}\right)$ is not a dominant strategy for bidder $i=1,2$.

Proof. We prove that $b_{1}=v_{1}\left(t_{1}, t_{1}\right)$ is not a dominant strategy for bidder 1 . First, we show that there is a set of types $I \in \mathcal{T}_{1}$ with $\mu_{1}(I)>0$, such that for every $t_{1} \in I$ bidding according $b_{1}\left(t_{1}\right)=v_{1}\left(t_{1}, t_{1}\right)$ has strictly negative payoff for bidder 1. By assumption we conclude that there is a set $B \in \mathcal{T}_{2}$ with $\mu_{2}(B)>0$ such that the map $v_{1}(\bar{t}, \cdot): T_{2} \rightarrow[\underline{a}, \bar{a}]$ is strictly increasing on $B$. Therefore, for every $t_{2} \in B \backslash\{\bar{t}\}$ we have

$$
v_{1}\left(\bar{t}, t_{2}\right)<v_{1}(\bar{t}, \bar{t})
$$


also for every $t_{2} \in T_{2}$ we know that

$$
v_{1}\left(\bar{t}, t_{2}\right) \leq v_{1}(\bar{t}, \bar{t})
$$

Therefore,

$$
\int_{T_{2}} v_{1}\left(\bar{t}, t_{2}\right) \mu_{2}\left(d t_{2}\right)<v_{1}(\bar{t}, \bar{t})=b_{1}(\bar{t}) .
$$

Let $y\left(t_{1}\right)=\int_{T_{2}} v_{1}\left(t_{1}, t_{2}\right) \mu_{2}\left(d t_{2}\right)$. Choose any $\widetilde{b}_{2} \in\left(y(\bar{t}), b_{1}(\bar{t})\right)$. Note that the maps $y$ and $b_{1}$ are strictly increasing, hence invertible. Therefore, $b_{1}^{-1}\left(\widetilde{b}_{2}\right)<\bar{t}<$ $y^{-1}\left(\widetilde{b}_{2}\right)$. Let $I=\left(b_{1}^{-1}\left(\widetilde{b}_{2}\right), \bar{t}\right]$, which is a nonempty interval of $T_{1}$ with $\mu_{1}(I)>0$. One can easily verify that for every $t_{1} \in I$ we have $y\left(t_{1}\right)<\widetilde{b}_{2}<b_{1}\left(t_{1}\right)$.

Suppose bidder 2 bids $\widetilde{b}_{2}$ for every $t_{2} \in T_{2}$ and bidder 1 bids according to $b_{1}\left(t_{1}\right)$. If $t_{1} \in I$, then bidder 1 wins the auction, since $\widetilde{b}_{2}<b_{1}\left(t_{1}\right)$ and his expected payoff for every $t_{1} \in I$ is

$$
\int_{T_{2}}\left(v_{1}\left(t_{1}, t_{2}\right)-\widetilde{b}_{2}\right) \mu_{2}\left(d t_{2}\right)=y\left(t_{1}\right)-\widetilde{b}_{2}<0 .
$$

In the case that $t_{1} \notin I$, the value of the map $y\left(t_{1}\right)$ strictly decreases when compared to the case $t_{1} \in I$. Consequently, even if bidder 1 wins the auction $y\left(t_{1}\right)-\widetilde{b}_{2}$ remains strictly negative. Hence, we have

$$
\int_{b_{1}\left(t_{1}\right)>\widetilde{b}_{2}} \int\left(v_{1}\left(t_{1}, t_{2}\right)-\widetilde{b}_{2}\right) \mu_{2}\left(d t_{2}\right) \mu_{1}\left(d t_{1}\right)<0 .
$$

Now, let $\widetilde{b}_{1}\left(t_{1}\right)=\underline{a}$ and $\widetilde{b}_{2}\left(t_{2}\right)=\widetilde{b}_{2}$. Moreover, let $\gamma_{1}, \widetilde{\gamma}_{1}$, and $\widetilde{\gamma}_{2}$ be the induced distributional strategies respectively by $b_{1}, \widetilde{b}_{1}$, and $\widetilde{b}_{2}$. Then, we have

$$
\Pi_{1}\left(\gamma_{1}, \widetilde{\gamma}_{2}\right)<\Pi_{1}\left(\widetilde{\gamma}_{1}, \widetilde{\gamma}_{2}\right)
$$

in which $\Pi_{1}\left(\widetilde{\gamma}_{1}, \widetilde{\gamma}_{2}\right)=0$ as bidder 1 always loses the auction by bidding $\widetilde{b}_{1}\left(t_{1}\right)=\underline{a}$. This shows that $b_{1}=v_{1}\left(t_{1}, t_{1}\right)$ is not a dominant strategy for every bidder 1 . The argument for bidder 2 is similar.

Notice that in the case when $\phi(t)=t$, for every $t \in[\underline{t}, \bar{t}]$, the distributional perfect BNE in which each player $i$ bids $b_{i}\left(t_{i}\right)=v_{i}\left(t_{i}, t_{i}\right)$ is a symmetric BNE. Moreover, if we assume that

$$
\frac{\partial v_{i}}{\partial t_{i}}\left(t_{1}, t_{2}\right)>\frac{\partial v_{j}}{\partial t_{i}}\left(t_{1}, t_{2}\right)
$$


for $j \neq i$, then $\left(b_{1}, b_{2}\right)$, in which $b_{i}=v_{i}\left(t_{i}, t_{i}\right)$ for every bidder $i$, is an efficient equilibrium. ${ }^{14}$ According to Krishna [23], this condition is called single crossing condition and ensures that the bidder with higher type has higher ex post valuation. Another interesting case of Proposition 4.5.1 is when $\Lambda$ is a common value auction, i.e. when $v_{1}\left(t_{1}, t_{2}\right)=v_{2}\left(t_{1}, t_{2}\right)$. In this case, the condition $v_{i}(t, \phi(t))=v_{i}(\phi(t), t)$, for $i=1,2$, holds for every increasing bijection $\phi$. Therefore, in common value auctions for every increasing bijection $\phi$, the strategy profile $\left(b_{1}, b_{2}\right)$ is distributional perfect.

In private value Vickrey auctions, each bidder has a dominant strategy that forms a distributional perfect BNE and leads to an efficient outcome. This observation motivates us to study the relation between distributional perfection and efficiency in our setting:

Remark. In the auction $\Lambda$ with single crossing condition, if $\phi(t) \geq \phi^{-1}(t)$, for every $t \in[\underline{t}, \bar{t}]$, then the strategy profile $\left(b_{1}, b_{2}\right)$ is efficient. The argument is as follows: Suppose that $x, y$ are the actual types that bidders 1 and 2 receive respectively and $x>y$. Then, the single crossing condition implies that bidder 1's ex post valuation is strictly larger than bidder 2's, that is $v_{1}(x, y)>v_{2}(x, y)$. Now, if $\phi(y) \geq \phi^{-1}(y)$, then we have

$$
b_{1}(x)=v_{1}(x, \phi(x))>v_{1}(y, \phi(y)) \geq v_{1}\left(y, \phi^{-1}(y)\right)=v_{2}\left(\phi^{-1}(y), y\right)=b_{2}(y) .
$$

This means that bidder 1 who had a higher ex post valuation for the object, wins the auction and is awarded the object.

Example 8.4 in Krishna [23] introduces the following class of discontinuous asymmetric strategy profiles for every $s_{1}, s_{2} \in[\underline{t}, \bar{t}]$ with $s_{1}<s_{2}{ }^{15}$ :

$$
\begin{aligned}
& \hat{b}_{1}\left(t_{1}\right)= \begin{cases}v_{1}\left(s_{2}, t_{1}\right) & \text { if } t_{1} \in\left[s_{1}, s_{2}\right] \\
v_{1}\left(t_{1}, t_{1}\right) & \text { otherwise, }\end{cases} \\
& \hat{b}_{2}\left(t_{2}\right)= \begin{cases}v_{2}\left(t_{2}, s_{1}\right) & \text { if } t_{2} \in\left[s_{1}, s_{2}\right] \\
v_{2}\left(t_{2}, t_{2}\right) & \text { otherwise. }\end{cases}
\end{aligned}
$$

He shows that if we assume the single crossing condition for the auction $\Lambda$, then $\left(\hat{b}_{1}, \hat{b}_{2}\right)$ is an ex post equilibrium in undominated strategies, moreover this equilibrium allocates the object inefficiently. In Proposition 4.5.3 we prove that distributional perfection at least rules out a subclass of these equilibria.

\footnotetext{
14 An outcome in any auction is called efficient if the winner is the one with the highest ex post valuation.

${ }^{15}$ Krishna presents the bid functions in terms of the opponent's valuations. We do this for each bidder in terms of his own valuations.
} 
Proposition 4.5.3 Suppose the auction $\Lambda$ satisfies the single crossing condition such that one of the followings holds:

(1) $v_{2}\left(s_{2}, s_{1}\right)<v_{2}\left(\underline{t}, s_{2}\right)$,

(2) $v_{1}\left(s_{2}, s_{1}\right)>v_{1}\left(s_{1}, \bar{t}\right)$.

Then, the ex post equilibrium $\left(\hat{b}_{1}, \hat{b}_{2}\right)$ is not distributional perfect.

Proof. First we discuss the case $v_{2}\left(s_{2}, s_{1}\right)<v_{2}\left(\underline{t}, s_{2}\right)$. Suppose by way of contradiction that $\left(\hat{b}_{1}, \hat{b}_{2}\right)$ is distributional perfect. Let $\hat{\gamma}_{i}$ be the distributional strategy induced by $\hat{b}_{i}$ and $\left(\gamma_{i}^{k}\right)_{k=1}^{\infty}$ be a sequence of completely mixed distributional strategies for each bidder $i=1,2$ that satisfies condition (i) and (ii) of Definition 4.3.1. Therefore, for bidder 2 and every $k$, there is a distributional strategy $\widetilde{\gamma}_{2}^{k} \in$ $B R_{2}\left(\gamma^{k}\right)$ such that $\rho^{w}\left(\gamma_{2}^{k}, \widetilde{\gamma}_{2}^{k}\right) \rightarrow 0$ as $k \rightarrow \infty$. Hence, by triangle inequality we conclude that $\rho^{w}\left(\widetilde{\gamma}_{2}^{k}, \hat{\gamma}_{2}\right) \rightarrow 0$ as $k \rightarrow \infty$. For every $k$, let $\beta_{1}^{k}, \beta_{2}^{k}$, and $\widetilde{\beta}_{2}^{k}$ be a corresponding behavioral strategy to $\gamma_{1}^{k}, \gamma_{2}^{k}$, and $\widetilde{\gamma}_{2}^{k}$ respectively. Also, let $\beta^{k}=\left(\beta_{1}^{k}, \beta_{2}^{k}\right)$. Then, by Lemma 4.6.1 we have $\widetilde{\beta}_{2}^{k}\left(t_{2}, \cdot\right) \in B R_{2}\left(t_{2}, \beta^{k}\right)$ for $\mu_{2^{-}}$ a.e. $t_{2} \in T_{2}$. By redefining $\widetilde{\beta}_{2}^{k}$, without loss of generality, we can assume that $\widetilde{\beta}_{2}^{k}\left(t_{2}, \cdot\right) \in B R_{2}\left(t_{2}, \beta^{k}\right)$ for every $t_{2} \in T_{2}$.

As $v_{2}\left(s_{2}, s_{1}\right)<v_{2}\left(\underline{t}, s_{2}\right)$, then there is a $\delta>0$ such that for every $t_{2} \in\left(s_{2}-\right.$ $\left.\delta, s_{2}\right]$, we have $v_{2}\left(t_{2}, s_{1}\right)<v_{2}\left(\underline{t}, t_{2}\right)$. Let bidder 2's type be $t_{2} \in\left(s_{2}-\delta, s_{2}\right]$. It is obvious that bidder 2's valuation is at least $v_{2}\left(\underline{t}, t_{2}\right)$. Since for every $k, \gamma_{1}^{k}$ is completely mixed, for every nonempty open set $B \subseteq A_{1}$, there is a type $t_{1} \in T_{1}$ such that $\beta_{1}^{k}\left(t_{1}, B\right)>0$. Therefore, bidder 1 might bid any bid $a_{1} \in A_{1}$ according to $\beta_{1}^{k}$. If $a_{1}<v_{2}\left(\underline{t}, t_{2}\right)$, then bidding $v_{2}\left(\underline{t}, t_{2}\right)$ is better than bidding $p_{2}<v_{2}\left(\underline{t}, t_{2}\right)$, since it increases the chance of winning. If $a_{1} \geq v_{2}\left(\underline{t}, t_{2}\right)$, then bidder 2 is indifferent between bidding any bid $p_{2} \leq v_{2}\left(\underline{t}, t_{2}\right)$. Therefore, bidder 2's best response against $\beta_{1}^{k}$, for every $k$, can not be any bid below $v_{2}\left(\underline{t}, t_{2}\right)$. Moreover, for every $t_{2} \in\left(s_{2}-\right.$ $\left.\delta, s_{2}\right]$, we have $\hat{b}_{2}\left(t_{2}\right)=v_{2}\left(t_{2}, s_{1}\right)<v_{2}\left(\underline{t}, t_{2}\right)$. Hence, if bidder 2 is given a type $t_{2} \in\left(s_{2}-\delta, s_{2}\right]$, then any of his best responses against $\beta_{1}^{k}$, for every $k$, is far from $\hat{b}_{2}\left(t_{2}\right)$. In particular, as for every $t_{2} \in T_{2}$ we have $\widetilde{\beta}_{2}^{k}\left(t_{2}, \cdot\right) \in B R_{2}\left(t_{2}, \beta^{k}\right)$, for every $k$, then for every $t_{2} \in\left(s_{2}-\delta, s_{2}\right]$ we have that $\rho^{w}\left(\hat{b}_{2}\left(t_{2}\right), \widetilde{\beta}_{2}^{k}\left(t_{2}, \cdot\right)\right)$ does not converge to zero when $k \rightarrow \infty$. Notice that $\hat{b}_{2}\left(t_{2}\right)$ is interpreted as a behavioral strategy that assigns probability 1 on $\hat{b}_{2}\left(t_{2}\right)$ for every $t_{2} \in T_{2}$.

Now, we show that there is $\varepsilon>0$ such that $\rho^{w}\left(\widetilde{\gamma}_{2}^{k}, \hat{\gamma}_{2}\right)>\varepsilon$ for every $k$. One can find $\varepsilon>0$ and $\lambda>0$ such that if for every $t_{2} \in\left(s_{2}-\delta, s_{2}\right]$ we define

$$
B_{t_{2}}=\left(\hat{b}_{2}\left(t_{2}\right)-\lambda, \hat{b}_{2}\left(t_{2}\right)+\lambda\right)
$$

then we have 
1. $s_{2}-\delta+\varepsilon<s_{2}-\varepsilon$,

2. $\mu_{2}\left(s_{2}-\delta+\varepsilon, s_{2}-\varepsilon\right)>\varepsilon$,

3. $\widetilde{\beta}_{2}^{k}\left(t_{2},\left(B_{t_{2}}\right)^{\varepsilon}\right)=0$.

Now, define $B=\left\{\left(t_{2}, a_{2}\right) \mid t_{2} \in\left(s_{2}-\delta+\varepsilon, s_{2}-\varepsilon\right], a_{2} \in B_{t_{2}}\right\}$. Clearly, $B$ is measurable. As $\hat{b}_{2}\left(t_{2}\right)\left(B_{t_{2}}\right)=1$ and $\hat{\beta}_{2}^{k}\left(t_{2},\left(B_{t_{2}}\right)^{\varepsilon}=0\right.$, we have

$$
\hat{\gamma}_{2}(B)=\int_{s_{2}-\delta+\varepsilon}^{s_{2}-\varepsilon} \hat{b}_{2}\left(t_{2}\right)\left(B_{t_{2}}\right) \mu_{2}\left(d t_{2}\right)=\mu_{2}\left(s_{2}-\delta+\varepsilon, s_{2}-\varepsilon\right),
$$

and

$$
\widetilde{\gamma}_{2}^{k}\left(B^{\varepsilon}\right)=\int_{s_{2}-\delta}^{s_{2}} \hat{\beta}_{2}^{k}\left(t_{2},\left(B_{t_{2}}\right)^{\varepsilon} \mu_{2}\left(d t_{2}\right)=0\right.
$$

Consequently,

$$
\hat{\gamma}_{2}(B)>\widetilde{\gamma}_{2}^{k}\left(B^{\varepsilon}\right)+\varepsilon,
$$

then $\rho^{w}\left(\widetilde{\gamma}_{2}^{k}, \hat{\gamma}_{2}\right)>\varepsilon$ for every $k$. This contradicts with the assumption $\rho^{w}\left(\widetilde{\gamma}_{2}^{k}, \hat{\gamma}_{2}\right) \rightarrow$ 0 as $k \rightarrow \infty$. Therefore, $\left(\hat{b}_{1}, \hat{b}_{2}\right)$ is not distributional perfect.

With an analogous argument for the case $v_{1}\left(s_{2}, s_{1}\right)>v_{1}\left(s_{1}, \bar{t}\right)$, the proof of the proposition is complete.

Conditions (1) and (2) in the above proposition are not very restrictive. For example, all auctions in which either $s_{1}=\underline{t}$ or $s_{2}=\bar{t}$, satisfy conditions (1) and (2) respectively. As an extreme case of this type of equilibria, we see that the BNE in which one bidder always bids $\bar{a}$ and the other bidder always bids $\underline{a}$ is not distributional perfect. This kind of equilibrium is called wolf and sheep equilibrium and is considered as a less intuitive one.

In the auction $\Lambda$, we change the condition (3) to be " $v_{i}$ is strictly increasing at $t_{i}$ and decreasing at $t_{j}$ for $j \neq i$ " and we call it $\left(3^{\prime}\right)$. Let $\Lambda^{\prime}$ be the auction that satisfies conditions (1), (2), $\left(3^{\prime}\right)$, and single crossing condition. In this auction $v_{1}(\underline{t}, \bar{t})=$ $v_{2}(\bar{t}, \underline{t})=\underline{a}$ and $v_{1}(\bar{t}, \underline{t})=v_{2}(\underline{t}, \bar{t})=\bar{a}$. Moreover, the class of BNEs corresponding to $\phi$ in Proposition 4.5.1 reduces to the single equilibrium where $\phi(t)=t$, that is $b_{1}\left(t_{1}\right)=v_{1}\left(t_{1}, t_{1}\right)$ and $b_{2}\left(t_{2}\right)=v_{2}\left(t_{2}, t_{2}\right)$. In the next proposition we show that this equilibrium is distributional perfect. To prove perfection in the following proposition we encounter more difficulties as compare to the proposition 4.5.1. The reason is that the bid functions of the bidders are not surjective which together with the possibility of making mistakes from the opponent, give the opportunity to the 
bidder to bid strictly higher than $b_{i}(1)=v_{i}(1,1)$ when he is from high type. The similar argument is valid if the bidder is from low type, i.e. he can bid strictly less than $b_{i}(0)=v_{i}(0,0)$.

Proposition 4.5.4 In the auction $\Lambda^{\prime}$, the strategy profile $\left(b_{1}, b_{2}\right)$ in which $b_{1}\left(t_{1}\right)=$ $v_{1}\left(t_{1}, t_{1}\right)$ and $b_{2}\left(t_{2}\right)=v_{2}\left(t_{2}, t_{2}\right)$ is a distributional perfect $B N E$.

Proof. Let $w(t)=v_{1}(t, t)=v_{2}(t, t)$, then the function $w:[\underline{t}, \bar{t}] \rightarrow[\underline{a}, \bar{a}]$ is strictly increasing but not surjective. It is easy to check that $\left(b_{1}, b_{2}\right)$ is a BNE. Now, we prove that it is distributional perfect. Let $\gamma_{1}$ be the distributional strategy induced by $b_{1}\left(t_{1}\right)$ and for every $k \in \mathbf{N}$ define $\gamma_{1}^{k}$ and $\beta_{1}^{k}$ as in the proposition 4.5.1. Let $p_{2}$ be a pure behavioral strategy for bidder 2 and $\sigma_{2}$ be the induced distributional strategy by $p_{2}$. Then, the expected payoff of bidder 2 choosing $\sigma_{2}$ against $\gamma_{1}^{k}$ conditioning on winning is:

$$
\Pi_{2}\left(\gamma_{1}^{k}, \sigma_{2}\right)=\int_{T_{2}} \Pi_{2}^{b}\left(\beta_{1}^{k}, p_{2} \mid t_{2}\right) \mu_{2}\left(d t_{2}\right),
$$

To maximize $\Pi_{2}\left(\gamma_{1}^{k}, \sigma_{2}\right)$, we maximize $\Pi_{2}^{b}\left(\beta_{1}^{k}, p_{2} \mid t_{2}\right)$ in the following three cases:

(1) $\underline{a} \leq p_{2} \leq w(\underline{t})$ :

$$
\Pi_{2}^{b}\left(\beta_{1}^{k}, p_{2} \mid t_{2}\right)=0+\varepsilon_{k} \int_{p_{2}>a_{1}}\left(v_{2}\left(t_{1}, t_{2}\right)-a_{1}\right) d \eta_{1}
$$

(2) $w(\underline{t}) \leq p_{2} \leq w(\bar{t})$ :

$$
\begin{aligned}
& \Pi_{2}^{b}\left(\beta_{1}^{k}, p_{2} \mid t_{2}\right)= \\
& \left(1-\varepsilon_{k}\right) \int_{\underline{t}}^{w^{-1}\left(p_{2}\right)}\left(v_{2}\left(t_{1}, t_{2}\right)-w\left(t_{1}\right)\right) \mu_{1}\left(d t_{1}\right)+\varepsilon_{k} \int_{p_{2}>a_{1}}\left(v_{2}\left(t_{1}, t_{2}\right)-a_{1}\right) d \eta_{1}
\end{aligned}
$$

(3) $w(\bar{t}) \leq p_{2} \leq \bar{a}$ :

$$
\begin{aligned}
& \Pi_{2}^{b}\left(\beta_{1}^{k}, p_{2} \mid t_{2}\right)= \\
& \left(1-\varepsilon_{k}\right) \int_{\underline{t}}^{\bar{t}}\left(v_{2}\left(t_{1}, t_{2}\right)-w\left(t_{1}\right)\right) \mu_{1}\left(d t_{1}\right)+\varepsilon_{k} \int_{p_{2}>a_{1}}\left(v_{2}\left(t_{1}, t_{2}\right)-a_{1}\right) d \eta_{1}
\end{aligned}
$$

Let $p_{21}^{k}, p_{22}^{k}$ and $p_{23}^{k}$ be the bid functions for bidder 2 that maximizes $\Pi_{2}^{b}\left(\beta_{1}^{k}, p_{2} \mid t_{2}\right)$ in the cases 1,2 and 3 respectively for every $t_{2}$. With a similar argument as in the proposition 4.5.1, we can see that $p_{22}^{k}$ converges to $b_{2}\left(t_{2}\right)=v_{2}\left(t_{2}, t_{2}\right)=w\left(t_{2}\right)$, as $k \rightarrow \infty$. Moreover, let

$$
M_{1}^{k}=\left\{t_{2} \in T_{2} \mid \Pi_{2}^{b}\left(\beta_{1}^{k}, p_{21}^{k} \mid t_{2}\right) \geq \Pi_{2}^{b}\left(\beta_{1}^{k}, p_{22}^{k} \mid t_{2}\right)\right\},
$$


for every $k \in \mathbf{N}$. It is obvious that $\mu_{2}\left(M_{1}^{k}\right) \rightarrow 0$ when $k \rightarrow \infty$. Also, let

$$
M_{2}^{k}=\left\{t_{2} \in T_{2} \mid \Pi_{2}^{b}\left(\beta_{1}^{k}, p_{23}^{k} \mid t_{2}\right) \geq \Pi_{2}^{b}\left(\beta_{1}^{k}, p_{22}^{k} \mid t_{2}\right)\right\} .
$$

Notice that $v_{2}\left(t_{1}, t_{2}\right)-w\left(t_{1}\right) \geq 0$ for every $t_{1} \leq t_{2}$ and negative otherwise, also we know that $w^{-1}\left(p_{22}^{k}\right) \rightarrow t_{2}$, as $k \rightarrow \infty$. Hence, for $\mu_{2}$-a.e. $t_{2} \in T_{2}$ and enough large $k$,

$$
\Pi_{2}^{b}\left(\beta_{1}^{k}, p_{23}^{k} \mid t_{2}\right) \leq \Pi_{2}^{b}\left(\beta_{1}^{k}, p_{22}^{k} \mid t_{2}\right)
$$

This means that $\mu_{2}\left(M_{2}^{k}\right) \rightarrow 0$ when $k \rightarrow \infty$. Therefore, for $\mu_{2}$-a.e. $t_{2} \in T_{2}$, $\Pi_{2}^{b}\left(\beta_{1}^{k}, p_{2} \mid t_{2}\right)$ is maximized when $p_{2}=b_{2}\left(t_{2}\right)=v_{2}\left(t_{2}, t_{2}\right)$ as $k \rightarrow \infty$. Вy a similar argument for bidder 2 the proof is complete.

In the auction $\Lambda^{\prime}$, there are many equilibria similar to $\left(\hat{b}_{1}, \hat{b}_{2}\right)$ in auction $\Lambda$ that can be rule out by distributional perfection. The examples of such equilibria can be found in Proposition 3.4.6 and Proposition 3.4.7 in chapter 3.

\subsection{Appendix}

Lemma 4.6.1 Let $\beta=\left(\beta_{1}, \ldots, \beta_{n}\right)$ be a behavioral strategy profile and $\gamma=$ $\left(\gamma_{1}, \ldots, \gamma_{n}\right)$ be the induced distributional strategy profile. Assume that the set of the best responses of player $i$ against behavioral strategy profile $\beta_{-i}$ is not empty. Then, if $\gamma_{i} \in B R_{i}(\gamma)$, then $\beta_{i}\left(t_{i}, \cdot\right) \in B R_{i}\left(t_{i}, \beta\right)$ for $\mu_{i}$-a.e. $t_{i} \in T_{i}$.

Proof. Suppose for every player $i$ that $\gamma_{i} \in B R_{i}(\gamma)$. Then, we have

$$
\Pi_{i}\left(\gamma_{i}, \gamma_{-i}\right) \geq \Pi_{i}\left(\widetilde{\gamma}_{i}, \gamma_{-i}\right)
$$

for every player $i$ 's distributional strategy $\widetilde{\gamma}_{i}$. Therefore, for every player $i$ 's behavioral strategy $\widetilde{\beta}_{i}$ we have

$$
\int_{T_{i}} \Pi_{i}^{b}\left(\beta_{i}, \beta_{-i} \mid t_{i}\right) \mu_{i}\left(d t_{i}\right) \geq \int_{T_{i}} \Pi_{i}^{b}\left(\widetilde{\beta}_{i}, \beta_{-i} \mid t_{i}\right) \mu_{i}\left(d t_{i}\right) .
$$

Moreover, by assumption, for every player $i$ there is a behavioral strategy $\zeta_{i}$ such that $\zeta_{i}\left(t_{i}, \cdot\right) \in B R_{i}\left(t_{i}, \beta\right)$ for every $t_{i} \in T_{i}$, which means having type $t_{i} \in T_{i}$

$$
\Pi_{i}^{b}\left(\zeta_{i}, \beta_{-i} \mid t_{i}\right) \geq \Pi_{i}^{b}\left(a_{i}, \beta_{-i} \mid t_{i}\right),
$$

for every $a_{i} \in A_{i}$. To prove that $\beta_{i}\left(t_{i}, \cdot\right)$ for $\mu_{i}$-a.e. $t_{i} \in T_{i}$ is a best response for every player $i$ against $\beta_{-i}$, we show that $\Pi_{i}^{b}\left(\zeta_{i}, \beta_{-i} \mid t_{i}\right)=\Pi_{i}^{b}\left(\beta_{i}, \beta_{-i} \mid t_{i}\right)$ for $\mu_{i}$-a.e. $t_{i} \in T_{i}$. Suppose the opposite. First, let

$$
S_{i}=\left\{t_{i} \in T_{i} \mid \Pi_{i}^{b}\left(\zeta_{i}, \beta_{-i} \mid t_{i}\right)<\Pi_{i}^{b}\left(\beta_{i}, \beta_{-i} \mid t_{i}\right)\right\} .
$$


Clearly, $S_{i}$ is $\mu_{i}$-measurable. Suppose $\mu_{i}\left(S_{i}\right)>0$. Notice that given $t_{i} \in T_{i}$, for $\beta_{i}\left(t_{i}, \cdot\right)$-a.e. $a_{i} \in A_{i}$ we have

$$
\Pi_{i}^{b}\left(\beta_{i}, \beta_{-i} \mid t_{i}\right)=\Pi_{i}^{b}\left(a_{i}, \beta_{-i} \mid t_{i}\right) .
$$

Then, given $t_{i} \in S_{i}$, for $\beta_{i}\left(t_{i}, \cdot\right)$-a.e. $a_{i} \in A_{i}$ we conclude that

$$
\Pi_{i}^{b}\left(\zeta_{i}, \beta_{-i} \mid t_{i}\right)<\Pi_{i}^{b}\left(a_{i}, \beta_{-i} \mid t_{i}\right)
$$

which is a contradiction with optimality of $\zeta_{i}$ for every type. Second, let

$$
S_{i}=\left\{t_{i} \in T_{i} \mid \Pi_{i}^{b}\left(\zeta_{i}, \beta_{-i} \mid t_{i}\right)>\Pi_{i}^{b}\left(\beta_{i}, \beta_{-i} \mid t_{i}\right)\right\}
$$

and suppose $\mu_{i}\left(S_{i}\right)>0$. Therefore, we have

$$
\int_{S_{i}} \Pi_{i}^{b}\left(\zeta_{i}, \beta_{-i} \mid t_{i}\right) \mu_{i}\left(d t_{i}\right)>\int_{S_{i}} \Pi_{i}^{b}\left(\beta_{i}, \beta_{-i} \mid t_{i}\right) \mu_{i}\left(d t_{i}\right) .
$$

Since $\zeta_{i}\left(t_{i}, \cdot\right)$ is a best response for every type $t_{i} \in T_{i}$ against $\beta_{-i}$, for every $a_{i} \in A_{i}$ we have

$$
\Pi_{i}^{b}\left(\zeta_{i}, \beta_{-i} \mid t_{i}\right) \geq \Pi_{i}^{b}\left(a_{i}, \beta_{-i} \mid t_{i}\right)
$$

thus

$$
\int_{T_{i} \backslash S_{i}} \Pi_{i}^{b}\left(\zeta_{i}, \beta_{-i} \mid t_{i}\right) \mu_{i}\left(d t_{i}\right) \geq \int_{T_{i} \backslash S_{i}} \Pi_{i}^{b}\left(\beta_{i}, \beta_{-i} \mid t_{i}\right) \mu_{i}\left(d t_{i}\right) .
$$

Consequently,

$$
\int_{T_{i}} \Pi_{i}^{b}\left(\zeta_{i}, \beta_{-i} \mid t_{i}\right) \mu_{i}\left(d t_{i}\right)>\int_{T_{i}} \Pi_{i}^{b}\left(\beta_{i}, \beta_{-i} \mid t_{i}\right) \mu_{i}\left(d t_{i}\right) .
$$

This is a contradiction with (4.1). Overall, we showed that if there is a subset of $T_{i}$ on which the expected payoff of player $i$, playing according to $\zeta_{i}$ is different from playing according to $\beta_{i}$, the measure of that subset is zero. In other words, for every $i$ there exists a set $S_{i} \in \mathcal{T}_{i}$ with $\mu_{i}\left(S_{i}\right)=0$ such that for every $t_{i} \in T_{i} \backslash S_{i}$ we have $\beta_{i}\left(t_{i}, \cdot\right) \in B R_{i}\left(t_{i}, \beta\right)$. This completes the proof.

In the lemma 4.6.2, we use the following metrics: Take $t, s \in T$ and $a, b \in A$. Define

$$
d_{T}(t, s)=\left(\sum_{i=1}^{n} d_{T_{i}}^{2}\left(t_{i}, s_{i}\right)\right)^{\frac{1}{2}} \quad, \quad d_{A}(a, b)=\left(\sum_{i=1}^{n} d_{A_{i}}^{2}\left(a_{i}, b_{i}\right)\right)^{\frac{1}{2}} .
$$

Moreover, let $d_{T \times A}((t, a),(s, b))=\left(d_{T}^{2}(t, s)+d_{A}^{2}(a, b)\right)^{\frac{1}{2}}$. 
Lemma 4.6.2 In Bayesian game $\Gamma$, for every player $i$ and every $\varepsilon>0$, there is a set $K \in \mathcal{T}$ with $\mu\left(K^{c}\right)<\varepsilon$ such that $\pi_{i}$ is continuous on $K \times$ A for every $i$.

Proof. Take $\varepsilon>0$ and $a \in A$. First we prove that there is a set $K \in \mathcal{T}$ with $\mu\left(K^{c}\right)<\varepsilon$ such that the function $\pi_{i}(\cdot, a): K \rightarrow \mathbf{R}$ is continuous. As $A$ is separable, it has a countable dense subset, say $B$. Hence, there exists a sequence $\left(a_{k}\right)_{k=1}^{\infty}$ in $B$ such that $a_{k} \neq a$, for every $k$, and $d_{A}\left(a_{k}, a\right) \rightarrow 0$ as $k \rightarrow \infty$. According to Lusin's Theorem for the collection of the functions $\left\{\pi_{i}\left(\cdot, a_{k}\right) \mid k=1,2, \ldots\right\}$ there is a compact set $E \in \mathcal{T}$ with $\mu\left(E^{c}\right)<\frac{\varepsilon}{2}$ such that $\pi_{i}\left(\cdot, a_{k}\right)$ is continuous on $E$ for every $k \in \mathbf{N}$. Moreover, by equicontinuity of payoffs there is a set $F \in \mathcal{T}$ with $\mu\left(F^{c}\right)<\frac{\varepsilon}{2}$ such that the collection of the functions $\left\{\pi_{i}(t, \cdot): A \rightarrow \mathbf{R} \mid t \in F\right\}$ is equicontinuous. Let $K=E \cap F$. It is clear that $\mu\left(K^{c}\right)<\varepsilon$. Moreover, because $\pi_{i}(t, \cdot)$ is continuous in actions for every $t \in K$, the sequence of the functions $\left(\pi_{i}\left(\cdot, a_{k}\right)\right)_{k=1}^{\infty}$ converges pointwisely to $\pi_{i}(\cdot, a)$ as $k \rightarrow \infty$. It is easy to check that equicontinuity of payoffs and the fact that $d_{A}\left(a_{k}, a\right) \rightarrow 0$ as $k \rightarrow \infty$, implies that the sequence of functions $\left(\pi_{i}\left(\cdot, a_{k}\right)\right)_{k=1}^{\infty}$ uniformly converges to $\pi_{i}(\cdot, a)$ on $K$ as $k \rightarrow \infty$. Hence, $\pi_{i}(\cdot, a): K \rightarrow \mathbf{R}$ is continuous too.

Now, we prove that $\pi_{i}$ is continuous at $(t, a) \in K \times A$. We know that $\left\{\pi_{i}(t, \cdot) \mid\right.$ $t \in K\}$ is equicontinuous, then there is a $\delta_{1}>0$ such that if $d_{A}(a, b)<\delta_{1}$, we have $\left|\pi_{i}(t, a)-\pi_{i}(t, b)\right|<\frac{\varepsilon}{2}$ for every $t \in K$. Also, we know $\pi_{i}(\cdot, a)$ is continuous at $t \in$ $K$, so there is a $\delta_{2}>0$ such that if $d_{T}(t, s)<\delta_{2}$, we have $\left|\pi_{i}(t, a)-\pi_{i}(s, a)\right|<\frac{\varepsilon}{2}$. Let $\delta=\min \left\{\delta_{1}, \delta_{2}\right\}$. Then, for every $(s, b) \in K \times A$ with $d_{T \times A}((t, a),(s, b))<\delta$ we have $d_{A}(a, b)<\delta_{1}$ and $d_{T}(s, t)<\delta_{2}$. Consequently, we have

$$
\left|\pi_{i}(t, a)-\pi_{i}(s, b)\right|<\left|\pi_{i}(t, a)-\pi_{i}(s, a)\right|+\left|\pi_{i}(s, a)-\pi_{i}(s, b)\right|<\frac{\varepsilon}{2}+\frac{\varepsilon}{2}=\varepsilon .
$$

This proves that $\pi_{i}$ is continuous on $K \times A$.

Lemma 4.6.3 In Bayesian game $\Gamma$, for every player $i$ the function $\Pi_{i}: \Delta \rightarrow \boldsymbol{R}$ is continuous with respect to the weak metric on $\Delta$.

Proof. To prove that $\Pi_{i}$ is continuous at a distributional strategy profile $\gamma$, let $\left(\gamma^{k}\right)_{k=1}^{\infty}$ be a sequence of distributional strategy profiles converging to $\gamma$ with respect to the weak metric as $k \rightarrow \infty$. Now, by Lemma 4.6.2 we have the following:

For every $i$ and every $\delta>0$, there are a continuous and bounded function $v_{\delta}$ : $T \times A \rightarrow \mathbf{R}$ and a set $K \in \mathcal{T}$ such that $\mu\left(K^{c}\right)<\delta$, and $v_{\delta}=\pi_{i}$ on $K \times A$.

As $\mu\left(K^{c}\right)<\delta$, we have $\gamma^{k}\left(K^{c} \times A\right)<\delta$, for every $k$. Similarly, $\gamma\left(K^{c} \times A\right)<\delta$. Moreover, because $v_{\delta}$ and $\pi_{i}$ are bounded, there is an $M>0$ such that $\left|\pi_{i}-v_{\delta}\right| \leq$ 
$M$. Hence, for every $k$ we have

$$
\int_{T \times A}\left|\pi_{i}-v_{\delta}\right| f(t) d \gamma^{k}=\int_{K^{c} \times A}\left|\pi_{i}-v_{\delta}\right| f(t) d \gamma^{k} \leq M \cdot \delta .
$$

Similarly,

$$
\int_{T \times A}\left|\pi_{i}-v_{\delta}\right| f(t) d \gamma=\int_{K^{c} \times A}\left|\pi_{i}-v_{\delta}\right| f(t) d \gamma \leq M \cdot \delta .
$$

Furthermore, as $f$ is $\hat{\mu}$-integrable, there is a sequence $\left(f_{\ell}\right)_{\ell=1}^{\infty}$ of bounded and continuous functions such that $\int_{T}\left|f(t)-f_{\ell}(t)\right| \hat{\mu}(d t) \rightarrow 0$, as $\ell \rightarrow \infty$. Now, we have

$$
\begin{aligned}
\left|\Pi_{i}\left(\gamma^{k}\right)-\Pi_{i}(\gamma)\right| & =\left|\int \pi_{i} f(t) d \gamma^{k}-\int \pi_{i} f(t) d \gamma\right| \\
& \leq \int\left|\pi_{i}-v_{\delta}\right| f(t) d \gamma^{k}+\int\left|f(t)-f_{\ell}(t)\right| v_{\delta} d \gamma^{k} \\
& +\left|\int v_{\delta} f_{\ell}(t) d \gamma^{k}-\int v_{\delta} f_{\ell}(t) d \gamma\right| \\
& +\int\left|f(t)-f_{\ell}(t)\right| v_{\delta} d \gamma+\int\left|\pi_{i}-v_{\delta}\right| f(t) d \gamma \\
& \leq M \delta+\int\left|f(t)-f_{\ell}(t)\right| v_{\delta} d \gamma^{k} \\
& +\left|\int v_{\delta} f_{\ell} d \gamma^{k}-\int v_{\delta} f_{\ell} d \gamma\right| \\
& +\int\left|f(t)-f_{\ell}(t)\right| v_{\delta} d \gamma+M \delta
\end{aligned}
$$

Hence, $\left|\Pi_{i}\left(\gamma^{k}\right)-\Pi_{i}(\gamma)\right|$ converges to zero when $k \rightarrow \infty$, $\ell \rightarrow \infty$, and $\delta \rightarrow 0$. This proves that $\Pi_{i}$ is continuous at $\gamma$. 



\section{Mathematical tools}

\section{Basic concepts of measure theory}

A semi-ring $\mathcal{S}$ is a collection of subsets of a set $X$ satisfying

1. $\emptyset \in \mathcal{S}$,

2. If $E, F \in \mathcal{S}$ then $E \cap F \in \mathcal{S}$,

3. If $E, F \in \mathcal{S}$ then there exists a countable collection $\left(G_{i}\right)_{i \in I}$ of pairwise disjoint sets in $\mathcal{S}$ such that $E \backslash F=\bigcup_{i \in I} G_{i}$.

A measure on a semi-ring $\mathcal{S}$ is a function $\mu: \mathcal{S} \rightarrow[0, \infty)$ such that

1. $\mu(\emptyset)=0$,

2. ( $\sigma$-additivity) For every countable collection $\left(A_{i}\right)_{i \in I}$ of pairwise disjoint sets in $\mathcal{S}$ with $\bigcup_{i \in I} A_{i} \in \mathcal{S}$ we have

$$
\mu\left(\bigcup_{i \in I} A_{i}\right)=\sum_{i \in I} \mu\left(A_{i}\right)
$$

A $\sigma$-field $\Sigma$ is a collection of subsets of a set $X$ satisfying

1. $X \in \Sigma$,

2. If $E \in \Sigma$ then $X \backslash E \in \Sigma$,

3. If $\left(E_{i}\right)_{i \in I}$ is a countable collection of sets in $\Sigma$ then $\bigcup_{i \in I} E_{i} \in \Sigma$.

The pair $(X, \Sigma)$ is called a measurable space.

A metric on a nonempty set $X$ is a function $d: X \times X \rightarrow[0, \infty)$ such that for all $x, y, z \in X$ we have 
1. $d(x, y)=0 \Leftrightarrow x=y \quad$ (identity of indiscernibles)

2. $d(x, y)=d(y, x) \quad$ (symmetry)

3. $d(x, z) \leq d(x, y)+d(y, z) \quad$ (triangle inequality).

The ordered pair $(X, d)$ is called a metric space. For $X \subseteq \mathbf{R}$ we always consider the standard Euclidean metric given by $d(x, y)=|x-y|$. The distance $d(x, B)$ between a point $x \in X$ and a nonempty set $B \subseteq X$ is defined as

$$
d(x, B)=\inf \{d(x, y) \mid y \in B\}
$$

and if $B=\emptyset$, then $d(x, B)=\infty$. The $\varepsilon$-neighborhood of $B$ is denoted by $B^{\varepsilon}=$ $\{x \in X \mid d(x, B)<\varepsilon\}$. A set $U \subseteq X$ is called open if for every $x \in U$ there is an $\varepsilon>0$ such that

$$
\{y \in X \mid d(x, y)<\varepsilon\} \subseteq U .
$$

A set $F \subseteq X$ is called closed if its complement $X \backslash F$ is open, and a set $C \subseteq X$ is called compact if, for every collection $\left\{U_{\alpha} \mid \alpha \in A\right\}$ of open sets such that $C \subseteq \cup_{\alpha \in A} U_{\alpha}$, it holds that $A$ has a finite subset $A^{\prime}$ such that $C \subseteq \cup_{\alpha \in A^{\prime}} U_{\alpha}$. Every compact set in a metric space is closed as well. The topology on $X$ induced by metric $d$ is the collection of all open sets, and the Borel $\sigma$-field $\Sigma$ on $X$ is the smallest $\sigma$ field that contains all open sets. A measure on $(X, \Sigma)$ is a function $\mu: \Sigma \rightarrow[0, \infty)$ such that $\mu(\emptyset)=0$ and $\mu$ is $\sigma$-additive. A measure $\mu$ is called a probability measure if $\mu(X)=1$. A probability measure is called completely mixed if it assigns strictly positive weight to every nonempty open set. For a metric space $(X, d)$, the set of probability measures on the Borel $\sigma$-field on $X$ is denoted by $\Delta(X)$. A probability measure $\mu \in \Delta(X)$ is completely mixed if $\mu(U)>0$ for every nonempty open subset $U$ of $X$. Moreover, define

$$
\operatorname{supp}(\mu)=\{x \in X \mid \text { for everey open set } G \text { if } x \in G \text { then } \mu(G)>0\} .
$$

Therefore, for every completely mixed probability measure, we have $\operatorname{supp}(\mu)=X$.

The weak (Prokhorov) metric $\rho^{w}$ on $\Delta(X)$ is defined for every $\mu, \nu \in \Delta(X)$ by

$$
\rho^{w}(\mu, \nu)=\inf \left\{\varepsilon>0 \mid \forall B \in \Sigma: \mu(B) \leq \nu\left(B^{\varepsilon}\right)+\varepsilon \text { and } \nu(B) \leq \mu\left(B^{\varepsilon}\right)+\varepsilon\right\}
$$

and the strong metric by

$$
\rho^{s}(\mu, \nu)=\sup \{|\mu(B)-\nu(B)| \mid B \in \Sigma\} .
$$

Note that for every $\mu$ and $\nu, \rho^{w}(\mu, \nu) \leq \rho^{s}(\mu, \nu)$. This easily follows from the observation that for every $B \in \Sigma$, both $\mu(B)-\nu\left(B^{\varepsilon}\right)$ and $\nu(B)-\mu\left(B^{\varepsilon}\right)$ are smaller 
than or equal to $|\mu(B)-\nu(B)|$ and therefore smaller than or equal to $\rho^{s}(\mu, \nu)$ as well.

The weak (strong) topology on $\Delta(X)$ is the topology induced by the weak (strong) metric. The inequality $\rho^{w}(\mu, \nu) \leq \rho^{s}(\mu, \nu)$ implies that the weak topology is included in the strong topology. If $X$ is compact, then so is $\Delta(X)$ with respect to $\rho^{w}$ (cf. Prokhorov [39] and Parthasarathy [38]). Further, compactness and sequential compactness are equivalent for metric spaces. ${ }^{16}$

Let $\mu_{n} \in \Delta(X)$ for every $n \in \mathbb{N}$ and let $\mu \in \Delta(X)$. It is known that if the sequence $\mu_{n}$ converges to $\mu$ with respect to $\rho^{w}$, then $\int_{X} f(x) \mu_{n}(d x)$ converges to $\int_{X} f(x) \mu(d x)$ for every bounded and continuous function $f: X \rightarrow \mathbb{R}$. Conversely, if $X$ is separable and $\int_{X} f(x) \mu_{n}(d x)$ converges to $\int_{X} f(x) \mu(d x)$ for every bounded and Lipschitz function $f: X \rightarrow \mathbb{R}$, then $\mu_{n}$ converges to $\mu$ with respect to $\rho^{w}$.

Let $\left(X_{1}, \Sigma_{1}\right),\left(X_{2}, \Sigma_{2}\right), \ldots,\left(X_{n}, \Sigma_{n}\right)$ be measurable spaces. The product $\sigma$ field $\otimes_{i=1}^{n} \Sigma_{i}$ on $\times_{i=1}^{n} X_{i}$ is defined to be the smallest $\sigma$-field that contains all the sets in $\times_{i=1}^{n} \Sigma_{i}$. Let $\mu_{i}$ be a measure on $\left(X_{i}, \Sigma_{i}\right)$, for every $1 \leq i \leq n$, and $\mu$ be a measure on the semi-ring $\times_{i=1}^{n} \Sigma_{i}$ defined by

$$
\mu\left(A_{1} \times \ldots \times A_{n}\right)=\prod_{i=1}^{n} \mu_{i}\left(A_{i}\right)
$$

for every $A_{i} \in \Sigma_{i}, 1 \leq i \leq n$. The product measure $\mu^{*}$ is the extension of the measure $\mu$ to the measurable space $\left(\times_{i=1}^{n} X_{i}, \otimes_{i=1}^{n} \Sigma_{i}\right)$ via the formula

$\mu^{*}(E)=\inf \left\{\sum_{k=1}^{\infty} \mu\left(\times_{i=1}^{n} A_{i}^{k}\right) \mid E \subseteq \bigcup_{k=1}^{\infty}\left(\times_{i=1}^{n} A_{i}^{k}\right), A_{i}^{k} \in \Sigma_{i}, 1 \leq i \leq n, \forall k \in \mathbf{N}\right\}$

According to Carathéodory's theorem, this extension is unique.

Let $\left(X, \Sigma_{1}\right),\left(Y, \Sigma_{2}\right)$ be two measurable spaces. A function $f: X \longrightarrow Y$ is called measurable if $f^{-1}(B) \in \Sigma_{1}$ for every $B \in \Sigma_{2}$.

\section{Semi-product measures}

Definition 4.6.4 Let $\left(X, \Sigma_{1}\right)$ and $\left(Y, \Sigma_{2}\right)$ be two measurable spaces, $\mu$ be a measure on $\left(X, \Sigma_{1}\right)$ and $\tau: X \times \Sigma_{2} \rightarrow[0, \infty)$ be a bounded function with the following properties :

\footnotetext{
${ }^{16}$ Throughout the dissertation we use several results from real analysis without further mention. All of the results we use are well-known. A good reference for the relevant theory is Aliprantis and Border [1].
} 
1. the section function $\tau(x, \cdot): \Sigma_{2} \rightarrow[0, \infty)$ is a measure on $\left(Y, \Sigma_{2}\right)$ for every $x \in X$,

2. the section function $\tau(\cdot, B): X \rightarrow[0, \infty)$ is a measurable function for every $B \in \Sigma_{2}$.

Let $\lambda$ be a measure on the semi-ring $\Sigma_{1} \times \Sigma_{2}$ defined by

$$
\lambda(A \times B)=\int_{A} \tau(x, B) \mu(d x),
$$

for every $A \times B \in \Sigma_{1} \times \Sigma_{2}$. The unique extension of the measure $\lambda$ by the formula (4.2) to the product $\sigma$-field $\Sigma_{1} \otimes \Sigma_{2}$ is denoted by $\lambda^{*}$ and is called the semi-product measure corresponding to $\tau$ and $\mu$.

Note that $\lambda$ is well-defined, because the section function $\tau(\cdot, B)$ is bounded and measurable for every $B \in \Sigma_{2}$. The following theorem states the integral respect to $\lambda^{*}$ as iterated integrals respect to $\tau$ and $\mu$. Dudley states a sketch of the proof in [11].

Theorem 4.6.5 Let $\left(X, \Sigma_{1}\right)$ and $\left(Y, \Sigma_{2}\right)$ be two measurable spaces and let $\lambda^{*}$ be the semi-product measure corresponding to $\tau$ and $\mu$. Then, for every function $f$ : $X \times Y \rightarrow \boldsymbol{R}$ bounded and measurable with respect to the $\sigma$-field $\Sigma_{1} \otimes \Sigma_{2}$ we have

$$
\int_{X \times Y} f d \lambda^{*}=\int_{X} \int_{Y} f \tau(x, d y) \mu(d x) .
$$

In particular, $\lambda^{*}(E)=\int_{X} \tau\left(x, E_{x}\right) \mu(d x)$ for every $E \in \Sigma_{1} \otimes \Sigma_{2}$ where $E_{x}=$ $\{y \in Y \mid(x, y) \in E\}$.

Proof. Let $E \in \Sigma_{1} \otimes \Sigma_{2}$. We first show that $\lambda^{*}(E)=\int_{X} \tau\left(x, E_{x}\right) \mu(d x)$. Let $\mathcal{N}$ be the collection of all sets $E \in \Sigma_{1} \otimes \Sigma_{2}$ such that

(i). $x \mapsto \tau\left(x, E_{x}\right)$ is a measurable function, and

(ii). $\lambda^{*}(E)=\int_{X} \tau\left(x, E_{x}\right) \mu(d x)$.

We prove that $\mathcal{N}^{X}$ is a $\sigma$-field.

1. It is clear that $X \times Y \in \mathcal{N}$. Indeed, $x \mapsto \tau(x, Y)$ is measurable according to condition (2) of Definition 4.6.4, and $\lambda^{*}(X \times Y)=\lambda(X \times Y)=\int_{X} \tau(x, Y) \mu(d x)$.

2. We show that if $E \in \mathcal{N}$, then $E^{c} \in \mathcal{N}$.

(i). Since $x \mapsto \tau\left(x, E_{x}\right)$ is measurable and $\tau(x, Y)-\tau\left(x, E_{x}\right)=\tau\left(x, E_{x}^{c}\right)$, the map $x \mapsto \tau\left(x, E_{x}^{c}\right)$ is also measurable. 


$$
\begin{aligned}
(i i) . \lambda^{*}\left(E^{c}\right)=\lambda^{*}(X \times Y)-\lambda^{*}(E) & =\int_{X} \tau(x, Y) \mu(d x)-\int_{X} \tau\left(x, E_{x}\right) \mu(d x) \\
& =\int_{X}\left(\tau(x, Y)-\tau\left(x, E_{x}\right)\right) \mu(d x) \\
& =\int_{X} \tau\left(x, Y \backslash E_{x}\right) \mu(d x) \\
& =\int_{X} \tau\left(x, E_{x}^{c}\right) \mu(d x)
\end{aligned}
$$

3. We show that if $\left(E^{i}\right)_{i=1}^{\infty}$ is a countable collection of sets in $\mathcal{N}$, then $\bigcup_{i=1}^{\infty} E^{i} \in \mathcal{N}$ :

Without loss of generality we assume that $E_{1}, E_{2}, \ldots$ are pairwise disjoint, because otherwise we can consider the pairwise disjoint sets $E_{1}, E_{2} \backslash E_{1}, E_{3} \backslash\left(E_{1} \cup\right.$ $\left.E_{2}\right), \ldots$

$(i)$. Since $x \mapsto \tau\left(x, E_{x}^{i}\right)$ is measurable for every $i \in \mathbf{N}$, by the Monotone Convergence Theorem the map that assigns to $x$ the real number $\lim _{n \rightarrow \infty} \sum_{i=1}^{n} \tau\left(x, E_{x}^{i}\right)=$ $\sum_{i=1}^{\infty} \tau\left(x, E_{x}^{i}\right)=\tau\left(x, \bigcup_{i=1}^{\infty} E_{x}^{i}\right)$ is also measurable.

(ii). According to the Monotone Convergence Theorem we have

$$
\begin{aligned}
\lambda^{*}\left(\bigcup_{i=1}^{\infty} E^{i}\right)=\sum_{i=1}^{\infty} \lambda^{*}\left(E^{i}\right) & =\lim _{n \rightarrow \infty} \sum_{i=1}^{n} \lambda^{*}\left(E^{i}\right) \\
& =\lim _{n \rightarrow \infty} \sum_{i=1}^{n} \int_{X} \tau\left(x, E_{x}^{i}\right) \mu(d x) \\
& =\int_{X} \lim _{n \rightarrow \infty} \sum_{i=1}^{n} \tau\left(x, E_{x}^{i}\right) \mu(d x) \\
& =\int_{X} \tau\left(x, \bigcup_{i=1}^{\infty} E_{x}^{i}\right) \mu(d x) \\
& =\int_{X} \tau\left(x,\left(\bigcup_{i=1}^{\infty} E^{i}\right)_{x}\right) \mu(d x) .
\end{aligned}
$$

Hence, $\mathcal{N}$ is a $\sigma$-field. Furthermore, it is clear that for every $A \in \Sigma_{1}$ and $B \in \Sigma_{2}$, $A \times B \in \mathcal{N}$. Since the product $\sigma$-field $\Sigma_{1} \otimes \Sigma_{2}$ is the smallest $\sigma$-field that contains $\Sigma_{1} \times \Sigma_{2}$, we have $\Sigma_{1} \otimes \Sigma_{2}=\mathcal{N}$. Therefore, $\lambda^{*}(E)=\int_{X} \tau\left(x, E_{x}\right) \mu(d x)$ for every set $E \in \Sigma_{1} \otimes \Sigma_{2}$. 
Now, we complete the proof of the theorem. We just proved that for every set $E \in \Sigma_{1} \otimes \Sigma_{2}$ we have

$$
\int_{X \times Y} \mathbf{1}_{E} d \lambda^{*}=\lambda^{*}(E)=\int_{X} \tau\left(x, E_{x}\right) \mu(d x)=\int_{X} \int_{Y} \mathbf{1}_{E_{x}} \tau(x, d y) \mu(d x) .
$$

Hence, it is clear that for any bounded and measurable step function $\phi$ on $X \times Y$ we have

$$
\int_{X \times Y} \phi d \lambda^{*}=\int_{X} \int_{Y} \phi_{x} \tau(x, d y) \mu(d x)=\int_{X} \int_{Y} \phi \tau(x, d y) \mu(d x),
$$

where $\phi_{x}(y)=\phi(x, y)$. Let $f: X \times Y \rightarrow \mathbf{R}$ be a bounded and measurable function with respect to the $\sigma$-field $\Sigma_{1} \otimes \Sigma_{2}$. Then, there is a sequence of bounded and measurable step functions $\left\{\phi^{n}\right\}_{n=1}^{\infty}$ such that $\phi^{n} \uparrow f$ pointwisely. Thus, by the Monotone Convergence Theorem we have

$$
\begin{aligned}
\int_{X \times Y} f d \lambda^{*} & =\int_{X \times Y} \lim _{n \rightarrow \infty} \phi^{n} d \lambda^{*} \\
& =\lim _{n \rightarrow \infty} \int_{X \times Y} \phi^{n} d \lambda^{*} \\
& =\lim _{n \rightarrow \infty} \int_{X} \int_{Y} \phi^{n} \tau(x, d y) \mu(d x) \\
& =\int_{X} \int_{Y} \lim _{n \rightarrow \infty} \phi^{n} \tau(x, d y) \mu(d x) \\
& =\int_{X} \int_{Y} f \tau(x, d y) \mu(d x) .
\end{aligned}
$$

\section{Fixed point theorems}

Let $X$ and $Y$ be two metric spaces. A correspondence between $X$ and $Y$ is a map from the elements of $X$ to the collection of all subsets of $Y$. So, for every $x \in X$, we have $F(x) \subseteq Y$. A correspondence $F$ between $X$ and $Y$ is upper-hemicontinuous if for every $x \in X$ and every sequence $\left(x_{n}\right)_{n=1}^{\infty}$ in $X$ converging to $x$ and every sequence $\left(y_{n}\right)_{n=1}^{\infty}$ in $Y$ converging to $y$ with $y_{n} \in F\left(x_{n}\right)$ it holds that $y \in F(x)$. A point $x \in X$ is called a fixed point for a correspondence $F: X \rightarrow X$ when $x \in F(x)$. We use the next theorem, Kakutani fixed point theorem, to prove the existence of weakly glof perfect equilibrium in chapter 2 .

Theorem 4.6.6 (Kakutani's fixed point theorem) Let $X$ be a non-empty, compact and convex subset of an Euclidean space. Let $F: X \rightarrow X$ be an upper-hemicontinuous correspondence such that $F(x)$ is non-empty, closed and convex for all $x \in X$. Then F has a fixed point. 
The following fixed point theorem is used to prove the existence of distributional perfect equilibrium in chapter 4. This theorem is derived from Glicksberg [13].

Theorem 4.6.7 Let $S$ be a nonempty compact and convex subset of a locally convex Hausdorff space. Let $F: S \rightarrow S$ be an upper hemicontinuous correspondence with nonempty and convex values. Then, $F$ has a fixed point. 



\section{Bibliography}

[1] Aliprantis CD, and Border KC (2006): Infinite Dimensional Analysis, a hitchhiker's guide. Springer-Verlag Publishers.

[2] Andersson O, Argenton C, and Weibull JW (2010): Robustness to Strategic Uncertainty. Center DP 2010-70.

[3] Athey S (2001): Single Crossing Properties and the Existence of Pure Strategy Equilibria in Games of Incomplete Information. Econometrica 69, 861-889

[4] Aumann RJ (1964): Mixed and Behavior Strategies in Infinite Extensive Games. Advances in Game Theory, Annals of Mathematical Studies 52, Princeton University Press, Princeton, N.J. 627-650.

[5] Balder E (2002): A Unifying Pair of Cournot-Nash Equilibrium Existence Results. Journal of Econoimc Theory 102, 437-470

[6] Billingsley P (1968): Convergence of Probability Measures. Wiley Series in Probability and Mathematical Statistics, John Wiley \& Sons.

[7] Carbonell-Nicolau O (2010): Essential Equilibria in Normal-form Games. Journal of Economic Theory, 145, 421-431.

[8] Carlsson H, and van Damme E (1993): Global games and equilibrium selection. Econometrica, 61, 989-1018.

[9] Compte O, and Jehiel P (2007): Auctions and Information Acquisition: sealed bid or dynamic formats. The Rand Journal of Economics, 38, 355-372.

[10] Crémer J, Spiegel Y, and Zheng CZ (2009): Auctions with Costly Information Acquisition. Economic Theory, 38, 41-72.

[11] Dudley RM (2002): Real Analysis and Probability. Press Syndicate of the University of Cambridge. 
[12] Dunford N, Schwartz JT (1957): Linear Operators, Part I: General Topology. New York: John Wiley and Sons.

[13] Glicksberg I (1952): A Further Generalization of Kakutani's Fixed Point Theorem with Application to Nash Equilibrium Points. Proc. Nat. Acad. Sci. U.S.A. 38 170-172.

[14] Govindan S, and Wilson R (2008): Metastable Equilibria. Mathematics of Operations Research, 33, 787-820.

[15] Hillas J (1990): On the Definition of the Strategic Stability of Equilibria. Econometrica, 58, 1365-1391.

[16] Jackson MO, Simon LK, Swinkels JM and Zame WR (2002): Communication and Equilibrium in Discontinuous Games of Incomplete Information. Econometrica 70, 1711-1740.

[17] Jackson MO, Simon LK, Swinkels JM and Zame WR (2004): Corrigendum to "Communication and Equilibrium in Discontinuous Games of Incomplete Information”. Econometrica 72, 1927-1929.

[18] Jiang Jia-He (1962): Essential Fixed Points of the Multivalued Mappings. Scientia Sinica 11, 293-298

[19] Kalai E, Samet D (1984): Persistent Equilibria in Strategic Games. International Journal of Game Theory, 13, 129-144.

[20] Kim T, Yannelis NC (1997): Existence of Equilibrium in Bayesian Games with Infinitely Many Players. Jounnal of Economic Theory 77, 330-353

[21] Kohlberg E, and Mertens JF (1986): On the Strategic Stability of Equilibria. Econometrica, 54, 1003-1037.

[22] Kreps D, and Wilson R (1982): Sequential Equilibria. Econometrica, 50, 863894.

[23] Krishna V (2002): Auction Theory. Academic Press.

[24] Mallozzi L, Pusillo L, Tijs S (2008): Approximate Equilibria for Bayesian Games. J. Math. Anal. Appl. 343, 10981102.

[25] Mcadams D (2003): Isotone Equilibrium in Games of Incomplete Information. Econometrica 71, 11911214. 
[26] Meirowitz A (2003): On the Existence of Equilibria in Bayesian Games with Non-finite Type and Action Spaces. Economics Letters 78, 213218.

[27] Méndez-Naya L, García-Jurado I, and Cesco JC (1995): Perfection of Nash Equilibria in Continuous Games. Mathematical Social Sciences, 29, 225-237.

[28] Mertens JF (1989): Stable Equilibria - A Reformulation. Part I: Definitions and basic properties. Mathematics of Operations Research 14, 575-625.

[29] Mertens JF (1991): Stable Equilibria - A Reformulation. Part II: Discussion of the definition and further results. Mathematics of Operations Research, 16, 694-753.

[30] Mertens JF (2003): Ordinality in Non- cooperative Games. International Journal of Game Theory. 32, 387-430.

[31] Mertens JF, Zamir S (1985): Formulation of Bayesian Analysis for Games with Incomplete Information. International Journal of Game Theory 14, 1-29.

[32] Milgrom PR (1981): Rational Expectations, Information Aquisition, and Competitive Bidding. Econometrica 49, 921-943.

[33] Milgrom PR, Weber RJ (1985): Distributional Strategies for Games with Incomplete Information. Mathematics of Operations Research 10, 619-632.

[34] Morris S, and Shin HS (2003): Global Games: theory and applications. Advances in economics and econometrics, M Dewatripont, L Hansen, and S Turnovsky (eds), Cambridge University Press, NY.

[35] Myerson RB (1978): Refinements of the Nash equilibrium concept. International Journal of Game Theory 7, 73-80.

[36] Nash JF (1950): Equilibrium Points in $n$-Person Games. Proceedings from the National Academy of Science USA, 36, 48-49.

[37] Nash JF (1951): Noncooperative Games. Annals of Mathematics, 54:286-295.

[38] Parthasarathy KR (1967): Probability Measures on Metric Spaces. Academic Press, New York - London, 1967.

[39] Prokhorov YV (1956): Convergence of Random Processes and Limit Theorems in Probability Theory, Theory of Probability and Its Applications, 1, 157214. 
[40] Reny PJ (2011): On the Exictance of Monotone Pure Strategy Equilibrium in Bayesian Games. Econometrica 79, 499-553.

[41] Selten R (1975): Reexamination of the Perfectness Concept for Equilibrium Points in Extensive Games. International Journal of Game Theory, 4, 25-55.

[42] Simon LK and Stinchcombe MM (1995): Equilibrium Refinement for Infinite Normal-form Games. Econometrica, 63, 1421-1443.

[43] van Damme E (1996): Stability and Perfection of Nash Equilibria. SpringerVerlag Publishers.

[44] Vermeulen AJ, and MJM Jansen (1997): On the Invariance of Solutions of Finite Games. Mathematical Social Sciences, 33, 251-267.

[45] V.Zandt T, Vives X (2007): Monotone Equilibria in Bayesian Games of Strategic Complementarities. Jounnal of Economic Theory, 134,339-360.

[46] Wagner DH (1977): Survey of Measurable Selection Theorems. Siam J. Control and Optimization, 15, 5, 859-903.

[47] Wu Wen-Tsün and Jiang Jia-He (1962): Essential Equilibrium Points of $n$ Person Non-cooperative Games. Scientia Sinica, 11, 1307-1322.

[48] Yu J (1999): Essential Equilibria of $n$-Person Non-cooperative Games. Journal of Mathematical Economics, 31, 361-372. 


\section{Nederlandse Samenvatting}

Simon en Stinchcombe hebben in 1995 het begrip perfect evenwicht gedefinieerd voor spelen met oneindige compacte verzamelingen van acties. Zij onderscheidden twee hoofdlijnen voor de mogelijke definities van perfect evenwicht. De eerste lijn is gebaseerd op het begrip compleet gemengd evenwicht, waarmee het sterke en zwakke perfecte evenwicht zijn gedefinieerd. Deze aanpak kan worden opgevat als een rechtstreekse generalisatie van de oorspronkelijke "trembling hand" definitie van perfect evenwicht door Selten in 1975. De tweede lijn van definities, vaak aangeduid met de term "finitistic approach", maakt gebruik van de notie van het $\varepsilon$ perfecte evenwicht in eindige benaderingen van het oorspronkelijke oneindige spel. Het resulterende begrip wordt aangeduid met "limiet-van-eindig" perfect evenwicht. Simon en Stinchcombe bewezen dat de eerste lijn van definities alleen gebruik maakt van zogeheten limiet toelaatbare strategieën. Verder spraken ze het vermoeden uit dat de twee benaderingen niet vergelijkbaar waren.

In hoofdstuk 2 onderzoeken we de relaties tussen de verschillende soorten van perfecte evenwichten binnen het kader van spelen in strategische vorm met compacte actieruimten en continue uitbetalingsfuncties. Verder introduceren we een verbeterde versie van de "finitistic approach", aangeduid met " globaal-limiet-van-eindig" perfect evenwicht, en bewijzen het bestaan van zulke evenwichten. Ondanks het feit dat de finitistic approach zeer intuïtief aandoet, lijken onze resultaten-met name voorbeelden [3] en [4] -een serieuze kritiek op deze aanpak te impliceren. In het eerste voorbeeld selecteert elke variant van de finitistic approach een Nash evenwicht dat niet limiet toelaatbaar is. Het tweede voorbeeld beschrijft een compleet gemengd (dus automatisch trembling hand perfect) Nash evenwicht dat niet finitistisch perfect is. Verdere voorbeelden dienen ter illustratie van de relaties tussen de twee verschillende benaderingen van het perfecte evenwicht, en de relatie tot toelaatbaarheid en ongedomineerdheid van strategieën.

In zogeheten second price veilingen met particuliere waarderingen voor de bieders heeft iedere bieder een dominante strategie waarin hij zijn persoonlijke waardering 
voor het object biedt. Echter, in een second price veiling met incomplete informatie en onderling afhankelijke waarderingen hebben bieders niet altijd een dominante strategie, terwijl er veel evenwichten kunnen zijn in een dergelijke veiling. Dit creëert de noodzaak om een instrument te ontwerpen om in zulke zogeheten Bayesiaanse spelen de minder intuïtieve evenwichten uit te sluiten. Met dit doel voor ogen ontwikkelen we in hoofdstukken 3 en 4 het begrip trembling hand perfect evenwicht voor Bayesiaanse spelen met oneindige type en actie ruimten, ter verfijning van de verzameling Bayesiaanse Nash evenwichten.

In hoofdstuk 3 baseren we ons onderzoek op zogeheten gedrag strategiën. Een centraal oplossingsconcept is het begrip Bayesiaans Nash evenwicht (BNE), een directe generalisatie van het Nash evenwicht. Een BNE is een profiel van gedrag strategieën, een voor elke speler, zó, dat de strategie van een speler, gegeven zijn type, in verwachte waarde een beste antwoord is op de strategieën van de tegenspelers, waarbij in de verwachte waarde alle mogelijke typen van de tegenspelers in beschouwing moeten worden genomen. Anders gezegd, de beste-antwoord eigenschap van het evenwicht wordt op interim niveau geëist, als een speler al weet wat zijn type is. Ons doel in dit hoodfstuk is om een formele definitie te geven van perfect BNE in Bayesiaanse spelen, en de eigenschappen daarvan in kaart te brengen. Als eerste stap definiëren we perfectie voor een gedrag strategie, dus niet noodzakelijk voor een BNE. We beschouwen drie varianten van perfectie, die verschillen in het convergentiebegrip dat gehanteerd wordt over de type ruimten. Kort gezegd is het centrale idee om een gedrag strategie $\beta$ perfect te noemen als er een rij $\left(\beta^{k}\right)_{k=1}^{\infty}$ compeet gemengde strategieën bestaat zó, dat voor elke speler $i$ de afstand tussen $\beta_{i}^{k}$ en $\beta_{i}$ en de afstand tussen $\beta_{i}^{k}$ en de beste antwoorden tegen $\beta^{k}$ beide naar nul convergeren. Met convergentie bedoelen we hier convergentie in de actie ruimte met betrekking tot de zwakke metriek, en convergentie in de type ruimten in één van de volgende drie betekenissen: uniform voor alle typen, puntsgewijs voor elk type, en puntsgewijs voor bijna elk type. In het algemeen is een dergelijk profiel $\beta$ niet per sé een BNE. Als $\beta$ ook nog een BNE is, dan noemen we $\beta$ uniform-perfect, puntsgewijs-perfect, of b.o.-puntsgewijs perfect. We geven een gedetailleerde analyse van elk van deze drie versies van perfect BNE, en bespreken de relaties tussen deze begrippen. We beschouwen ook enkele speciale gevallen, zoals compactheid van type en actie ruimten, en continuiteit van de uitbetalingsfuncties.

In hoofdstuk 4 beschouwen we Bayesiaanse spelen met $n$ spelers waar de type ruimte van elke speler een separabele metrische ruimte is. Zodra spelers hun type kennen, dienen ze gelijktijdig een actie uit een compacte metrische ruimte te kiezen, waarna elke speler een uitbetaling ontvangt die af kan hangen van zowel de typen van 
de spelers als de acties die ze hebben gekozen. Zoals in Milgrom en Weber (1985) veronderstellen we dat de a priori kansverdeling op het product van de type ruimten absoluut continu is in relatie tot het product van de resulterende marginale kansen. We nemen ook aan dat de uitbetalingen equicontinu zijn. Milgrom en Weber (1985) bewijzen dat er in dit geval minstens een evenwicht in distributie strategieën bestaat. Aangezien het niet duidelijk is hoe spelers een distributie strategie dienen te spelen, definiëren we een analoog begrip voor gedrag strategieën. Een gedrag strategie wordt een distributie evenwicht genoemd als de resulterende distributie strategieën een evenwicht vormen.

Om het begrip trembling hand perfect evenwicht te generaliseren naar Bayesiaanse spelen, definiëren we in dit hoofdstuk het distributie perfect evenwicht als een gedrag strategie profiel wiens resulterende distributie strategie profiel voldoet aan de voorwaarden voor het trembling hand perfect evenwicht. We bewijzen dat voor bovenstaande klasse van Bayesiaanse spelen distributie perfectie een verfijning is van het distributie evenwicht. Ook bewijzen we dat een distributie perfect evenwicht bestaat, en dat de verzameling distributie perfecte evenwichten rijgesloten is in Tychnoff topologie, indien we de verzameling van kansmaten op de actie ruimten topologiseren met de sterke topologie.

We benadrukken dat de aanpak in hoofdstuk 3 om perfecte evenwichten te definiëren natuurlijker is dan de methode die we hanteren in hoofdstuk 4. Echter, om de theorie van perfectie te ontwikkelen, en met name om existentie van perfecte evenwichten te bewijzen, is het eenvoudiger om de tweede aanpak in hoofdstuk 4 te gebruiken dan de eerste in hoofdstuk 3.

Verder, in beide hoofdstukken, gebruiken de deze methoden in de context van symmetrische sealed-bid second-price veilingen met onderling afhankelijke waarderingen, en laten zien dat perfectie een specifiek type evenwicht selecteert, en de minder natuurlijke evenwichten uitsluit. Verder merken we op dat in deze veilingen de bieders geen dominante strategieën hebben, en dat er een groot aantal evenwichten in ongedomineerde strategieën bestaat.

Preciezer gezegd, in hoofdstuk 3 beschouwen we een second price veiling waarin voor elke $i=1,2$ de waardering voor bieder $i$ wordt gegeven door $v_{i}=5+t_{i}-\alpha t_{j}$, met $\alpha \in(0,1)$ en $j \neq i$. Er zijn veel BNEs in deze veiling, maar we laten zien dat perfectie een BNE $\beta$ selecteert die uniek is binnen een klasse van strategieën, en de minder natuurlijke evenwichten uitsluit. We benadrukken dat de keuze van de rij gedrag strategie profielen $\left(\beta^{k}\right)_{k=1}^{\infty}$ een subtiele zaak is, aangezien de meest voor de hand liggende kandidaat niet het gewenste resultaat geeft. 
In hoofdstuk 4 beschouwen we een symmetrische second price veiling met twee bieders waar de waardering van bieder $i$ voor het object af kan hangen van beide typen. Deze waardering wordt aangeduid met $v_{i}\left(t_{1}, t_{2}\right)$ en heeft de volgende eigenschappen:

(1) $v_{1}\left(t_{1}, t_{2}\right)=v_{2}\left(t_{2}, t_{1}\right)$.

(2) $v_{i}$ is continu differentieerbaar.

(3) $v_{i}$ is strikt stijgend in $t_{i}$ and stijgend in $t_{j}$ voor $j \neq i$.

We gebruiken distibutie perfectie in deze veiling om een kleinere verzameling evenwichten te verkrijgen, en de contra-intuïtieve evenwichten uit te sluiten. 


\section{Biography}

Elnaz Bajoori was born on September 18, 1980 in Zahedan, Iran. She did her undergraduate studies in pure mathematics at Iran University of Science and Technology, Tehran. She continued with a research Master in pure mathematics, mathematical analysis at Kharazmi University (Tarbiat Moallem University) in Tehran. She did her Master thesis on "fixed point theorems for set-valued functions with applications to game theory" which was a turning point for her to get interested in game theory and economics. She was a lecturer at Azad University of Saveh in Iran for four years, besides she was learning economics and game theory by self-studying and sitting in different economics classes.

She started her PhD program in September 2009 at Maastricht University under supervision of Prof. Dries Vermeulen and Dr. János Flesch. Her PhD research was on trembling hand perfect equilibrium in games with infinite action spaces. During her PhD she visited the Economics Departments of the University of Chicago, the University of Birmingham, and the University of Exeter. She has presented her research papers in several conferences and workshops such as International Conference on Game Theory in Stony Brook, Economic Theory Workshop of the University of Chicago, Fourth Congress of Game Theory in Istanbul, and seminar series in Mathematics Department of London School of Economics.

To pursue her academic life, Elnaz is going to take up a lectureship position at the University of Bath, Department of Economics from September 2013. 